\title{
Dual-Wavelength Polarization Independent Grating Coupler Design Based on Silicon-on-Insulator
}

\author{
by
}

\author{
Tianyi Hao
}

A thesis submitted to the Faculty of Graduate and Postdoctoral Affairs in partial fulfillment of the requirements for the degree of

Master of Applied Science

in

Electrical and Computer Engineering

Carleton University

Ottawa, Ontario

(C) 2020

Tianyi Hao 


\section{Abstract}

Silicon photonics has emerged as one of the most popular areas in integrated optics because of its compatibility with CMOS fabrication processes and its potential for low cost and mass production. To enable efficient fiber-to-chip coupling of light, one key component is a grating coupler. With an increasing demand for wavelength-division-

multiplexing systems in fiber-to-the-home network services, low-cost dual-band (O- and C-bands) chip transceivers are required.

In this thesis, the universal design methodology of conventional and subwavelength grating couplers has been presented. Firstly, a vertical incident wavelength splitting grating coupler that can split $1310 \mathrm{~nm}$ and $1550 \mathrm{~nm}$ wavelength light into two directions is shown. Afterward, a polarization splitting grating coupler that can work for both $\mathrm{C}$ - and $\mathrm{O}-$ band with a flexible incident angle is also demonstrated. In the last part of this thesis, a novel dual-band subwavelength polarization independent grating coupler works as a wavelength demultiplexer is proposed. It has transmissions of $\sim 30 \%$ and $3-\mathrm{dB}$ bandwidths of $80-100$ $\mathrm{nm}$ for both TE and TM polarizations of O- and C-band. 


\section{Acknowledgements}

I would like to thank my supervisor, professor Winnie N. Ye, for all the help and guidance she provided. She can always show the most up-to-date technology and theories to us and lead me to the right path of studying and researching.

I would also like to extend my gratitude to all other professors taught me, Dr. Jacques Albert, Dr. Derek McNamara Dr. Khaled Mnaymneh, and Dr. Ksenia Dolgaleva, for the knowledge they shared.

I would like to thank my colleagues Dr. Alejandro Sánchez-Postigo, Ruhul Fatin, Dusan Gostimirovic, and Fu Liu, for the advice and ideas they gave. Special thanks to Alejandro Sánchez-Postigo for his help in discussing and editing the papers.

Besides all the people help me during my study, I would also like to thank my guitar

for being part of my life. Many thanks to Mr. Makoto Shinkai, Mr. Nisio Isin, Falcom, and Square Enix for all the stories they created and presented that gave me strength and moved me.

Last but not least, I would like to thank my parents, Dabing Hao and Hengdi Zhu, for all the support they gave and always respecting my choices. 


\section{Table of Contents}

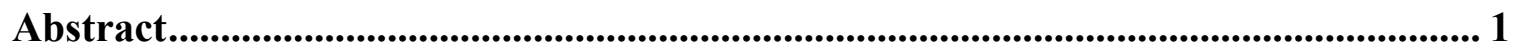

Acknowledgements ........................................................................................................................ 2

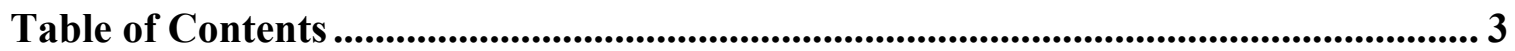

List of Tables ............................................................................................................................ 5

List of Figure ........................................................................................................................ 6

List of Appendices........................................................................................................................ 13

Chapter 1: Introduction ............................................................................................................ 14

$1.1 \quad$ Silicon Photonics ............................................................................................ 14

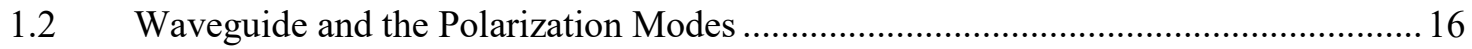

1.3 Coupling between Fiber and Nanophotonics Waveguide …………………………...2

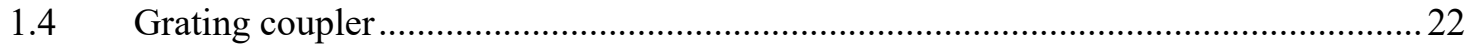

$1.5 \quad$ Polarization Independent Grating Couplers ……………………………………...2

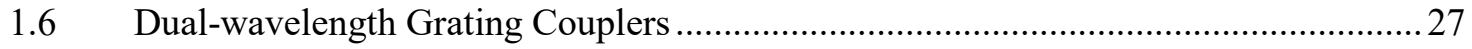

$1.7 \quad$ Thesis Objectives and Organization ......................................................................... 31

Chapter 2: Grating Theory and Numerical Methods........................................................ 33

$2.1 \quad$ Bragg Condition .................................................................................................... 33

2.2 Analysis of the Grating Performances..................................................................... 38

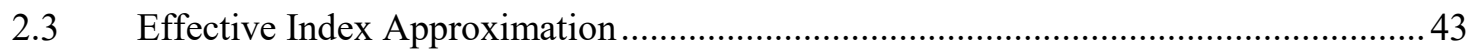

2.4 Finite Difference Time Domain Method ………………………………………..........51

Chapter 3: Vertical Incident Dual-wavelength Grating Coupler.................................. 56

3.1 Shallow Etched Grating Coupler Designed................................................................56

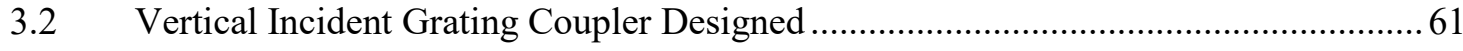

3.3 Combining of Two Wavelength Designs Grating Couplers......................................... 72 
Chapter 4: Dual-band Polarization Splitting Grating Coupler.

4.1 Polarization Splitting Grating Coupler Design .......................................................... 79

4.2 Dual-Band Polarization Splitting Grating Coupler.................................................... 81

Chapter 5: Dual-Wavelength Polarization Independent Grating Coupler .............. 86

5.1 Two-Dimensional Subwavelength Polarization Independent Grating Coupler ...........86

5.2 Dual Bands Polarization Independent Grating coupler ........................................... 91

5.3 Optimization and Tolerance Analysis of the Dual-Band Polarization Independent

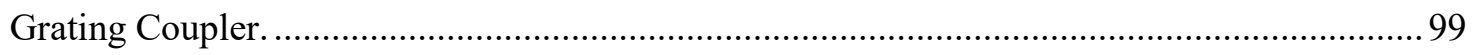

Chapter 6: Conclusion and Future Work........................................................... 105

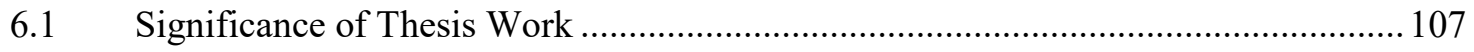

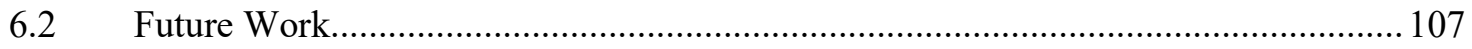

Appendices................................................................................................................................. 110

Bibliography or References................................................................................................ 126 


\section{List of Tables}

1. Table 1.1 State-of-art grating couplers work for a single wavelength...................... 24

2. Table 1.2 State-of-art polarization independent grating couplers .......................... 27

3. Table 1.3 State-of-art dual-wavelength grating couplers...................................... 30

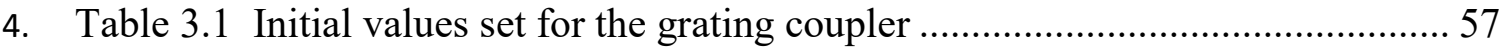

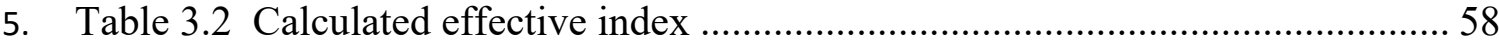

6. Table 3.3 Optimized vertical incident grating coupler parameters .......................... 71

7. Table 4.1 Design parameters for the four ports grating coupler ......................... 83

8. Table 5.1 Design parameters for the dual-band polarization independent grating

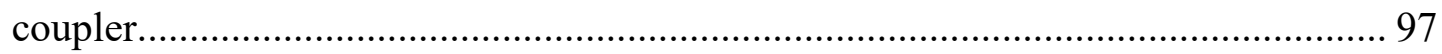

9. Table 5.2 Design parameters for the optimized dual-band polarization independent

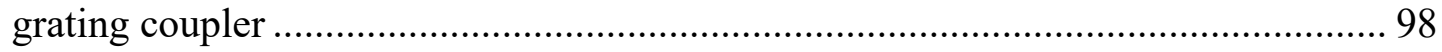

10. Table 5.2 The data of the polarization independent wavelength splitting grating coupler 


\section{List of Figure}

1. Figure 1.1 Side view of an SOI wafer's layer structure...................................... 16

2. Figure 1.2 Schematics of (a) slab waveguide (b) slab waveguide ......................... 17

3. Figure 1.3 Mode profiles of Electric field intensity of (a) $\mathrm{TE}_{0}$ and (b) $\mathrm{TM}_{0}, \mathrm{E}_{\mathrm{x}}$ field of (c) $\mathrm{TE}_{0}$ and (d) $\mathrm{TM}_{0}$, and $\mathrm{E}_{\mathrm{y}}$ field of (e) $\mathrm{TE}_{0}$ and (f) $\mathrm{TM}_{0}$

4. Figure 1.4 Cross-section diagram of (a) a single mode fiber and (b) a silicon waveguide under the same scale 20

5. Figure 1.5 Schematics of (a) butt coupling, (b) end-fire coupling, (c) prism coupling and (d) grating coupling 21

6. Figure 1.6 Schematics of (a) the shallow-etched grating coupler and (b) the side view of one period 22

7. Figure 1.7 Design schematics of (a) the first $2 \mathrm{D}$ grating coupler used as a polarization splitter [30] (b) the first 1D polarization splitter grating coupler [26] 25

8. Figure 1.8 (a) Proposed architectures of the gratings and its working theory [11]. (b) The coupling efficiency spectrum. 25

9. Figure 1.9 Proposed architectures of 2D polarization independent grating coupler [31]

10. Figure 1.10 Design schematics of (a) the wavelength splitting grating coupler with the same polarization [23] and (b) a dual-wavelength grating coupler with different polarizations [21] 28 
11. Figure 1.11 Design schematics of (a) the vertical incident wavelength splitting grating coupler done with inverse design and (b) the optimization with the different iteration

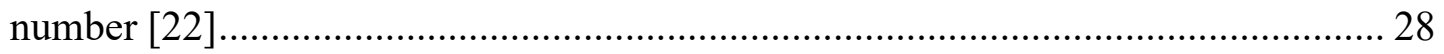

12. Figure 1.12 Design schematics of (a) the vertical incident wavelength splitting grating coupler done with inverse design [24] and (b) a dual-wavelength grating coupler made

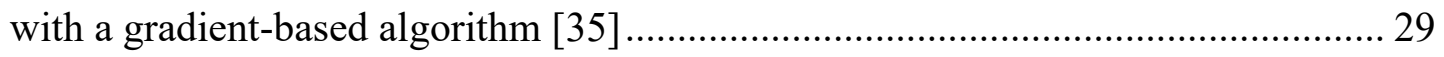

13. Figure 2.1 Schematic of the Bragg diffraction..................................................... 34

14. Figure 2.2 Schematics of (a) a one-dimensional grating coupler and (b) a crystalline solid approach grating coupler 35

15. Figure 2.3 Schematic Bragg condition of (a) the first-order and (b) the second-order $[55]$ 37

16. Figure 2.4 Schematic of the field distribution of the light coupled out from the uniform grating coupler [7]. 39

17. Figure 2.5 Schematic (a) dispersion diagram and (b) corresponding electric field profiles of a periodic slab waveguide for the three regimes of subwavelength-guided wave propagation, Bragg reflection, and radiation. Ref. [44] 40

18. Figure 2.6 schematic diagram of the diffractive field of an apodized grating coupler [7] 41

19. Figure 2.7 schematic diagram of (a) a classic focusing grating coupler [6] and (b) and apodized focusing grating coupler with a subwavelength structure [8] 42

20. Figure 2.8 schematic diagram of the waveguide structure for effective index analysis

21. Figure 2.9 schematic diagram of a 1-D planar waveguide 44 
22. Figure 2.10 The effective index versus thickness of an SOI platform structure........ 46

23. Figure 2.11 the Effective Index Method analysis steps for (a) a strip waveguide and (b) a rib waveguide.

24. Figure 2.12 Schematic diagram of a wave propagating perpendicular to the alternation media.

25. Figure 2.13 Schematic diagram of a wave propagating parallel to the alternation media

26. Figure 2.14 Schematic diagram of a waveguide structure with (a) a poor mesh size and (b) a fine mesh size. 53

27. Figure 2.15 Schematic diagram of a vertical incident grating coupler..... 54

28. Figure 3.1 The calculated and simulated effective index versus waveguide height.. 58

29. Figure 3.2 The incident angle versus period length for $1550 \mathrm{~nm}$ and $1310 \mathrm{~nm} \mathrm{TE}$ mode

30. Figure 3.3 The example grating coupler in Lumerical FDTD Solutions 60

31. Figure 3.4 (a)The transmission spectrum and (b) power profile of the example grating coupler. 60

32. Figure 3.5 Schematic diagram of a vertical incident grating coupler..... 62

33. Figure 3.6 Schematic Bragg condition of a vertical incident grating coupler 62

34. Figure 3.7 Schematic Bragg condition within the waveguide of the vertical incident grating coupler 63

35. Figure 3.8 Simulation transmission spectrum for a vertical incident grating coupler at operating wavelengths (a) $1310 \mathrm{~nm}$ and (b) $1550 \mathrm{~nm}$ 64 
36. Figure 3.9 Transmission spectrum for (a) left port and (b) right port with a fiber position shift..

37. Figure 3.10 Transmission spectrum for (a) left port and (b) right port with an incident angle sweep 66

38. Figure 3.11 Transmission spectrum of the vertical incident grating coupler with a duty cycle sweep 67

39. Figure 3.12 Transmission spectrum of the vertical incident grating coupler with a period length sweep 68

40. Figure 3.13 Transmission spectrums of the vertical incident grating coupler with (a) an etch-depth sweep or (b) a waveguide thickness sweep.....

41. Figure 3.14 Transmission spectrum of the vertical incident grating coupler with a sweep of the number of periods 70

42. Figure 3.15 Transmission spectrum of optimized vertical incident grating couplers design for (a) $1550 \mathrm{~nm}$ and (b) $1310 \mathrm{~nm}$ 72

43. Figure 3.16 Schematic diagram of the dual-wavelength vertical incident grating coupler. 73

44. Figure 3.17 Transmission spectrum of the dual-band vertical incident grating couplers at (a) $1550 \mathrm{~nm}$ and (b) $1310 \mathrm{~nm}$ 74

45. Figure 3.18 Transmission spectrums of vertical incident grating couplers design with (a) a combined width sweep and (b) a fiber position sweep. 75

46. Figure 3.19 Schematic diagram of the apodized dual-wavelength vertical incident grating coupler 76 
47. Figure 3. 20 Transmission spectrum of the apodized dual-wavelength vertical incident grating coupler 76

48. Figure 3.21 Coupling efficiency spectrum of the optimized dual-wavelength vertical incident grating coupler at (a) $1310 \mathrm{~nm}$ and (b) $1550 \mathrm{~nm}$ 77

49. Figure 4.1 The absolute value of the incident angle versus the period length based on the Bragg condition. 80

50. Figure 4.2 The schematic Bragg condition for a polarization splitting grating coupler 80

51. Figure 4.3 the schematic diagrams of (a) four ports grating coupler and its (b) top view, (c) side view from xz plane and (d) size view from yz plane for one period 81

52. Figure 4.4 the schematic diagrams of (a) four ports grating coupler and its (b) top view, (c) side view from xz plane and (d) size view from yz plane 83

53. Figure 4.5 The schematic diagram for the four ports grating coupler that works for (a) $1550 \mathrm{~nm}$ and (b) $1310 \mathrm{~nm}$ 84

54. Figure 4.6 The transmission spectrum of four ports grating coupler with (a) $1310 \mathrm{~nm}$ TE, (b) $1310 \mathrm{~nm} \mathrm{TM,} \mathrm{(c)} 1550 \mathrm{~nm} \mathrm{TE,} \mathrm{(d)} 1550 \mathrm{~nm}$ TM 84

55. Figure 5.1 the schematic diagrams of (a) a grating coupler with subwavelength squarehole structure and its (b) top view and (c) side view for one period 87

56. Figure 5.2 Calculation and simulation result of the effective index of the square-shape subwavelength structure. 88

57. Figure 5.3 The effective index versus $f f_{\mathrm{x}}$ and $f f_{\mathrm{y}}$ of (a) $1550 \mathrm{~nm}$ and (b) $1310 \mathrm{~nm}$ in a grating coupler with subwavelength square-shape-hole structure. 
58. Figure 5.4 The incident angle versus period length for a polarization independent grating coupler with square-shape holes. 90

59. Figure 5.5 The effective index versus $f f_{\mathrm{x}}$ and $f f_{\mathrm{y}}$ of $1550 \mathrm{~nm}$ and $1310 \mathrm{~nm}$ in a grating coupler with subwavelength square-shape-hole structure. 91

60. Figure 5.6 The $f f_{\mathrm{x}}$ versus $f f_{\mathrm{y}}$ that satisfy polarization independent for $1550 \mathrm{~nm}$ and $1310 \mathrm{~nm}$ in a grating coupler with subwavelength square-shape-hole structure....... 92

61. Figure 5.7 The effective index versus waveguide thickness for first, second, and third modes of $1550 \mathrm{~nm}$ and $1310 \mathrm{~nm}$. 93

62. Figure 5.8 The polarization independent design of $\mathrm{ff}_{\mathrm{x}}$ versus $\mathrm{ff}_{\mathrm{y}}$ for $(\mathrm{a})$ the calculation result and (b) comparison of simulation and calculation result. 93

63. Figure 5.9 (a) Schematic representation of the polarization-independent wavelengthsplitting subwavelength grating coupler. (b) Top view of one period. (c) Side view of one period. 95

64. Figure 5.10 The $f f_{\mathrm{x}}$ versus $f f_{\mathrm{y}}$ that satisfy polarization independent for $1550 \mathrm{~nm}$ and $1310 \mathrm{~nm}$ in a grating coupler with subwavelength square-shape-hole structure....... 96

65. Figure 5.11 The incident angle versus period length that satisfies Bragg condition for the dual-band polarization independent grating coupler design .....

66. Figure 5.12 Coupling efficiency as a function of the wavelength for (a) O-band [TE], (b) C-band [TE], (c) O-band [TM] and (d) C-band [TM].

67. Figure 5.13 (a) Schematic representation of the polarization-independent wavelengthsplitting subwavelength grating coupler. (b) Top view of one period. (c) Side view of one period. 100 
68. Figure 5.14 The $\mathrm{ff}_{\mathrm{x}}$ versus $\mathrm{ff}_{\mathrm{y}}$ that satisfy polarization independent for $1550 \mathrm{~nm}$ and $1310 \mathrm{~nm}$ in the dual-band polarization independent grating coupler design 101

69. Figure 5.15 Coupling efficiency of the dual-band polarization independent grating coupler for (a) O-band [TE], (b) C-band [TE], (c) O-band [TM] and (d) C-band [TM]. 102

70. Figure 5.16 Tolerance analysis of the designed dual-band polarization-insensitive wavelength-splitting grating coupler. (a) Central wavelength and (b) coupling efficiency deviation as a function of errors in the shallow-etched depth. (c) Central wavelength and (d) coupling efficiency deviation as a function of errors in the length $\left(\Delta \Lambda_{x} \mathrm{ff}_{x}\right)$ and width $\left(\Delta \Lambda_{y} \mathrm{ff}_{y}\right)$ of the grating teeth. 103

71. Figure 6.1 Schematic of a polarization and wavelength splitting grating coupler... 108

72. Figure 6.2 Schematics of a dual-wavelength grating coupler (a) without cladding and (b) with a specially designed cladding 108 


\section{List of Appendices}

Appendix A FDTD Scripts to Generate Grating Coupler ....................... 103

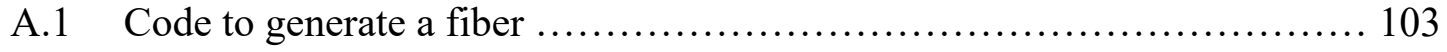

A.2 Code to generate a vertical incident dual-band grating coupler ............ 103

A.3 Code to generate a 3D grating coupler with square-shape holes ........... 103

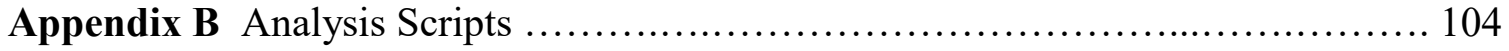

B.1 Matlab Code for 1-D Effective Index Approximation ..................... 104

B.2 Plot the incident angle versus period length from the Bragg condition ...... 104

B.3 Parameter sweep for 2-D grating coupler ........................... 104

B.4 Matlab Code for EMT Polarization Independent Calculation ............... 104

B.5 Matlab code for Dual-Band Polarization Independent incident angle and period

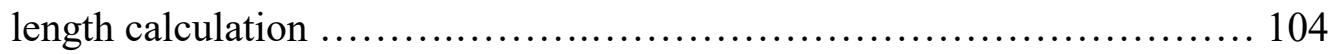




\section{Chapter 1:}

\section{Introduction}

\subsection{Silicon Photonics}

Silicon photonics has emerged as one of the most popular areas in photonics research since the late 1980s [1]. After decades of development of electronics that use microwave frequency, optics and photonics have attracted more and more researchers because of their higher frequency, broader bandwidth, shorter wavelength, lower transmission loss, and better sensitivity. Because of its low cost and CMOS compatibility, silicon has been widely used in most kinds of photonic devices - especially in photonic integrated circuits (PICs). Silicon also has a wide optic transparency range (1.1 $\mu \mathrm{m}$ to $7 \mu \mathrm{m})$ and an absorption spectrum in the visible range [2]. This allows silicon to be used in many areas such as communication, sensing, detecting, and photovoltaics.

In the information age, networks with a higher data rate and capacity are necessary. Replacing or combining electronic devices with photonic devices is one of the best choices to satisfy these requirements. Unlike electronics that need to use tens or hundreds of components to build a single device, silicon photonics can realize the same use with one or a few simple structures such as gratings, multimode interferometers (MMI), rings or discs, tapers, etc. Photonic devices can realize the same purposes, such as switching, filtering, modulation, transmission, and wavelength multiplexing/demultiplexing, as electric devices do by combining the basic structures of the optical waveguides. At the 
same time, photonic devices can work much faster than electronic devices. Optical networks that use the wavelength division multiplexing (WDM) system are the most popular communication systems [3]. The coarse wavelength division multiplexing (CWDM) with $20 \mathrm{~nm}$ channel separation has the advantages of low power consumption, low cost, small size, and temperature insensitivity. The dense wavelength division multiplexing (DWDM) is applied more widely than the CWDM. $0.4 \mathrm{~nm}$ or $0.8 \mathrm{~nm}$ channel separations that work though C-band $(1530 \mathrm{~nm}-1565 \mathrm{~nm})$ and L-band $(1565 \mathrm{~nm}-1625$ $\mathrm{nm}$ ) increase the data capacity of the network dramatically. A $0.4 \mathrm{~nm}$ channel separation means a $50 \mathrm{GHz}$ bandwidth, while a $0.8 \mathrm{~nm}$ channel separation means a $100 \mathrm{GHz}$ bandwidth. For example, an optic fiber with up to 96 DWDM wavelength channel separations can support and transmit data at a rate of $4000 \mathrm{GHz}$. By applying photonics technologies, a faster processing and computing communication system can be built.

Other than applications in the area of communication, silicon photonics is also widely used in sensing area, such as gas-sensing and bio-sensing. More importantly, the LiDAR (Light Radar) area has a great development potential [4]. Depending on the wavelength, LiDAR is used for different purposes such as facial recognition, autonomous cars, airborne surveying, and aerospace. Almost all the components in the LiDAR system can be built out of silicon except for the laser source. This is because the indirect bandgap of silicon makes it challenging to realize an active light source with silicon. Industries and researchers have done lots of research and development on low-cost and lossless power splitters, phase shifters, phase arrays, transmitters, and receivers. More and more applications used on smartphones, cars, and space have shown a market that is developing and continuing to grow. The high resolution, small device dimensions, and anti- 
interference ability make LiDAR perform better in many applications than traditional microwave radar.

Photovoltaics that converts the energy of daylight directly into electricity is another popular application of silicon photonics. Higher energy demand, coupled with severe environmental problems, requires cheaper and more efficient solar cells. In the future, photovoltaic applications could provide enough clean energy to everyone in the world.

Silicon also has many other useful properties. By applying stress on silicon, the Pockels effect or electro-optic effect can be used to build a modulator. Doped silicon has a strong plasma dispersion effect. Raman and Kerr effects can also be applied - the Raman effect can even realize an all-silicon laser design [5].

\subsection{Waveguide and the Polarization Modes}

In the optics and photonics area, a waveguide is a structure that guides light waves. There are two types of waveguides that are used the most frequently: the fiber waveguide and the planar waveguide. Silicon is the waveguide material used in silicon photonics.

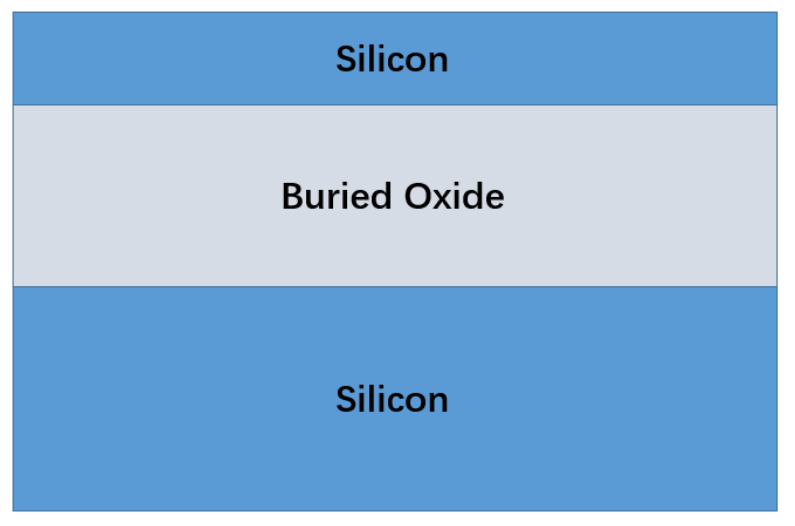

Figure 1.1 Side view of an SOI wafer's layer structure

The silicon-on-insulator (SOI) platform is an ideal technology for designing and building planar waveguide circuits. As figure 1.1 shows, an SOI wafer structure contains 
three layers. From bottom to top, these are the silicon substructure layer, the buried oxide (BOX) layer, and the silicon film layer. The top silicon layer is the functional wave-guiding layer. $220 \mathrm{~nm}$ is the most-used thickness of the silicon film layer because it is the bestworking thickness for $1550 \mathrm{~nm}$ single mode light. Usually, the silicon substructure used for mechanical support has a thickness larger than $100 \mu \mathrm{m}$. The BOX layer works as the insulator layer and has a $2 \mu \mathrm{m}$ to $3 \mu \mathrm{m}$ thickness. Silicon dioxide $\left(\mathrm{SiO}_{2}\right)$ is the most commonly used material for the BOX layer. Because of the oxidation characteristics of silicon, an additional $\mathrm{SiO}_{2}$ layer might be used as the cover layer on the waveguide. The huge refractive index difference between the silicon layer $\left(\mathrm{n}_{\mathrm{si}} \sim 3.46\right.$ at $\left.1550 \mathrm{~nm}\right)$ and the $\mathrm{SiO}_{2}\left(\mathrm{n}_{\mathrm{SiO} 2} \sim 1.44\right.$ at $\left.1550 \mathrm{~nm}\right)$ of the SOI leads to efficient light confinement in very compact devices.

For the planar waveguide, there are two representative geometries: the slab waveguide and the rib waveguide. These two waveguides are shown in Figure 1.2 (a) and (b), respectively.

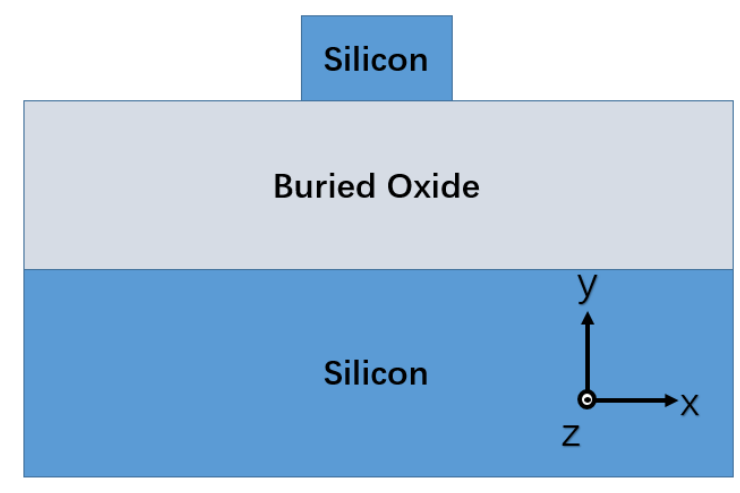

(a)

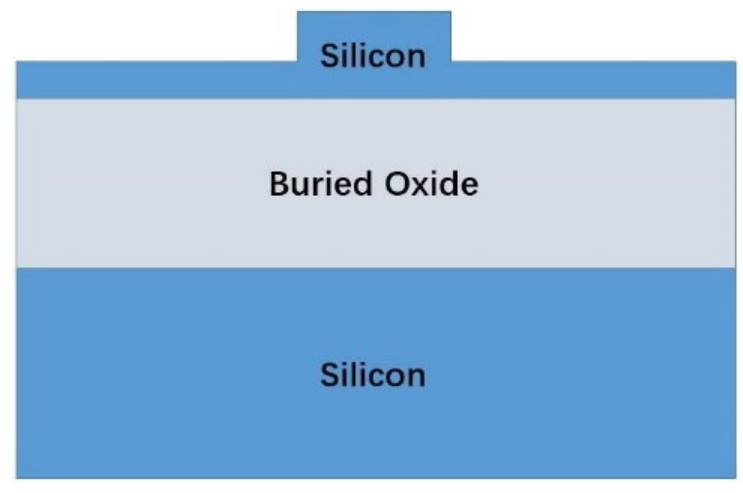

(b)

Figure 1.2 Schematics of (a) slab waveguide (b) slab waveguide

The slab waveguide is the most commonly used planar waveguide. In the rectangular-shaped waveguide, the light propagates along the z-axis. There are two types 
of mode existing: transverse electric (TE) modes and transverse magnetic (TM) modes. TE modes are defined as those modes having electric fields perpendicular to the plane of incidence, and TM modes are defined as those modes having magnetic fields perpendicular to the plane of incidence. In the coordinate system shown in Figure 1.2, the yz plane is defined as the plane of incidence.

In particular, the so-called $\mathrm{TE}_{\mathrm{m}}$ and $\mathrm{TM}_{\mathrm{n}}$ modes are not ideal $\mathrm{TE}$ and $\mathrm{TM}$ modes in 1-D. Because of the width limit (x-axis) of the waveguide in 2-D optical confinement, the modes guided in the waveguide are TE-like and TM-like modes.

For the propagating wave associated with electric and magnetic fields, the propagation constant $k$ (also known as wave vector $\beta$ ) of the guided modes is defined as:

$$
\mathrm{k}=\frac{\mathrm{d} \phi}{\mathrm{dz}}=\mathrm{nk}_{0}=\frac{2 \pi}{\lambda_{0}} \mathrm{n}
$$

Where $\phi$ is the phase of the propagating wave, $\mathrm{z}$ is the direction of propagation, $\mathrm{k}_{0}$ represents the propagating wave in free space, $\lambda_{0}$ denotes the wavelength of the operating light, and $\mathrm{n}$ is the material refractive index of the waveguide which can be written as

$$
\mathrm{n}=\sqrt{\varepsilon_{\mathrm{r}} \mu_{r}}=\frac{\mathrm{c}}{\mathrm{v}_{\mathrm{p}}}
$$

Where $\varepsilon_{\mathrm{r}}$ is the waveguide's relative permittivity, $\mu_{r}$ is its relative permeability, $\mathrm{c}$ is the speed of light in the vacuum, and $v_{p}$ is the phase velocity of the light in the medium. Importantly, refractive effective index is wavelength dependent and its value is decided by the waveguide material property. 


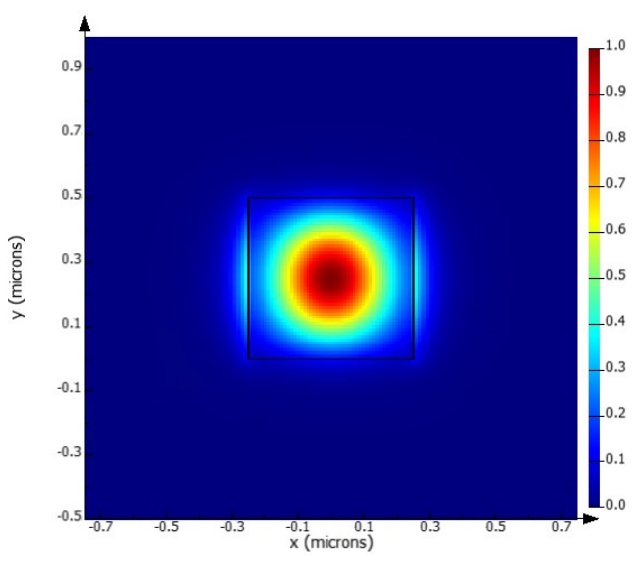

(a)

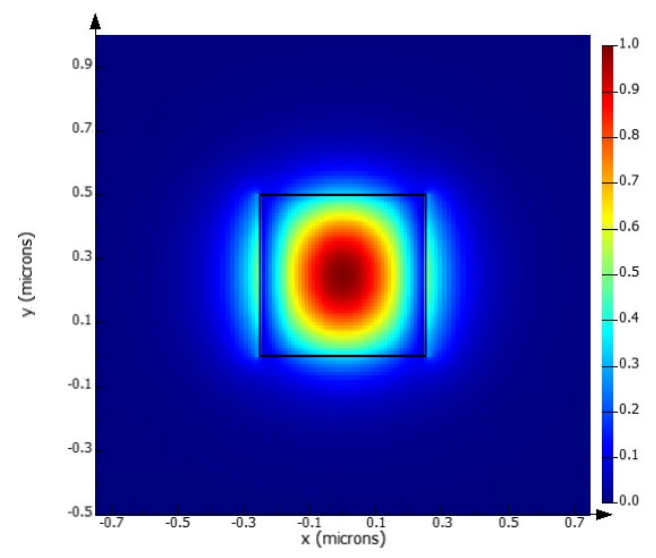

(c)

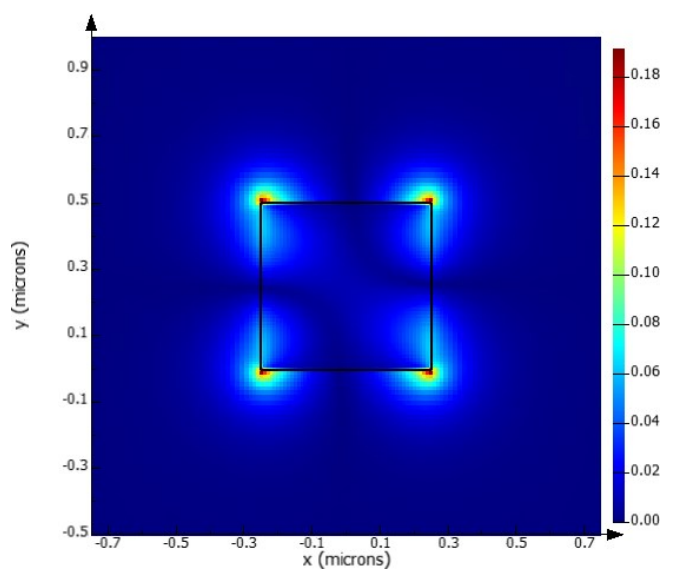

(e)

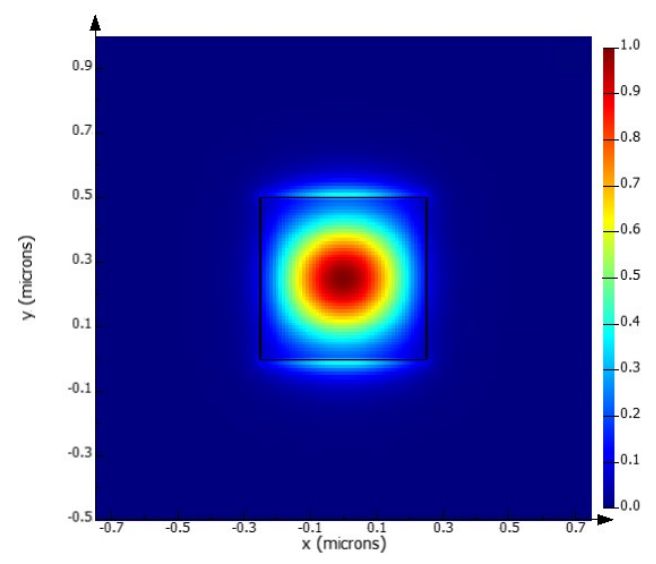

(b)

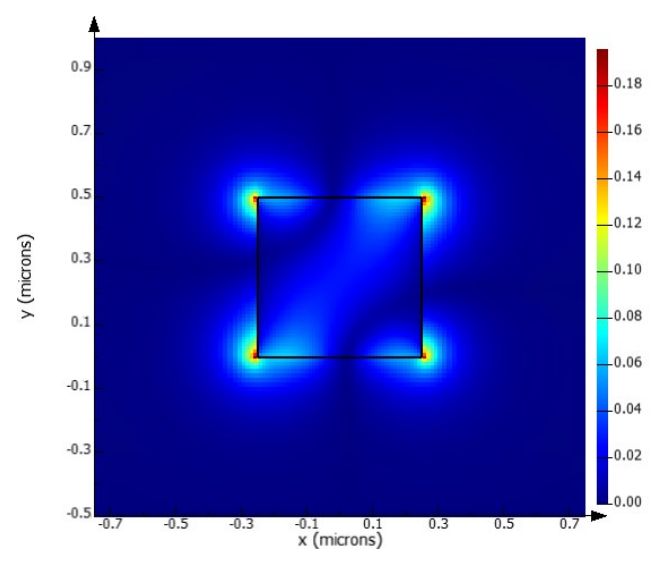

(d)

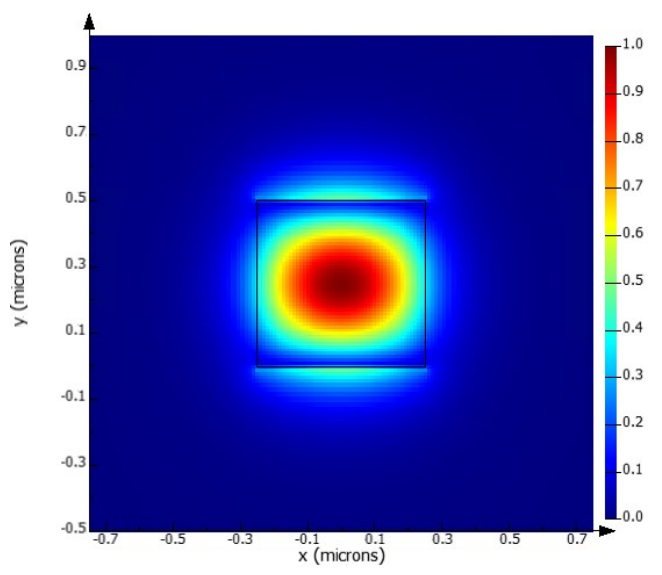

(f)

Figure 1.3 Mode profiles of Electric field intensity of (a) $\mathrm{TE}_{0}$ and (b) $T \mathrm{M}_{0}, \mathrm{E}_{\mathbf{x}}$ field of (c) $\mathrm{TE}_{0}$ and (d) $\mathrm{TM} \mathrm{M}_{0}$, and $\mathrm{E}_{\mathrm{y}}$ field of (e) $\mathrm{TE} \mathrm{E}_{0}$ and (f) $\mathrm{TM}$, 
Figure 1.3 shows the difference between the TE and TM. These two modes are referred to as $E_{x}$ or $E_{y}$ based on their maximum polarization direction. In other words, TE modes have a horizontal distributed intensity profile, and the TM modes have a vertical distributed intensity profile. While TE modes have strong $\mathrm{E}_{\mathrm{x}}\left(\right.$ and $\mathrm{H}_{\mathrm{y}}$ ) fields and weak $\mathrm{E}_{\mathrm{y}}$ (and $\mathrm{H}_{\mathrm{x}}$ ) fields, TM modes have strong $\mathrm{E}_{\mathrm{y}}\left(\right.$ and $\mathrm{H}_{\mathrm{x}}$ ) fields and weak $\mathrm{E}_{\mathrm{x}}\left(\right.$ and $\mathrm{H}_{\mathrm{y}}$ ) fields.

\subsection{Coupling between Fiber and Nanophotonics Waveguide}

The high index contrast of the silicon-on-insulator (SOI) platform leads to efficient light confinement in very compact devices, whose cross-section is much smaller than the diameter of conventional single-mode optical fibers. Typically, the silicon waveguide has a cross-section area of $220 \mathrm{~nm} x \sim 500 \mathrm{~nm}$, and a single mode fiber core has a diameter of $\sim 10 \mu \mathrm{m}$. The cross-section area of the fiber is almost 1000 times larger than the silicon waveguide. Figure 1.4 shows the size difference between a 9 - $\mu$ m-diameter fiber core and a $220 \mathrm{~nm} \times 1 \mu \mathrm{m}$ silicon waveguide.

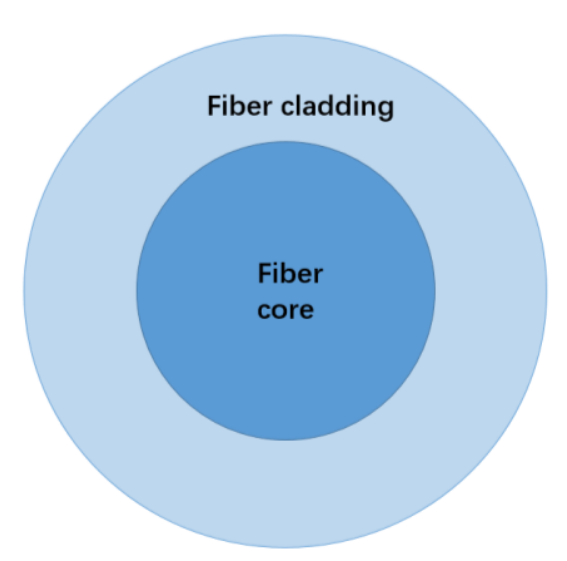

(a)
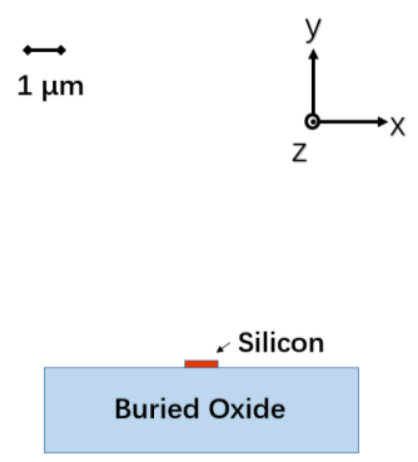

Figure 1.4 Cross-section diagram of (a) a single mode fiber and (b) a silicon waveguide under the same scale 
To enable the fiber-to-chip coupling of light, several techniques have been used. Figure 1.5 (a) and (b) show the schematics of edge coupling. The butt coupling can transmit the light directly into the waveguide. Because of the cross-section area difference between the fiber and the planer waveguide, the butt coupling would lose most of the light. The endfiber coupling adds a lens or uses a lensed fiber to focus light in a small region and increase the coupling efficiency (CE). However, these two coupling methods (known as edge couplings) still need to face the problems such as mismatching of field excitation and waveguide mode, reflection from the waveguide facet, or spatial misalignment. Antireflection coatings and tapers are used in the edge coupling to reduce the coupling loss.

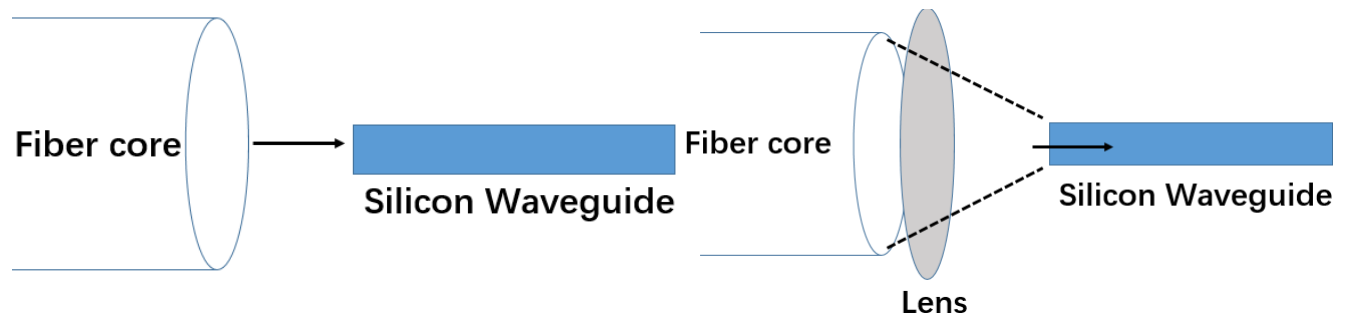

(a)

(b)

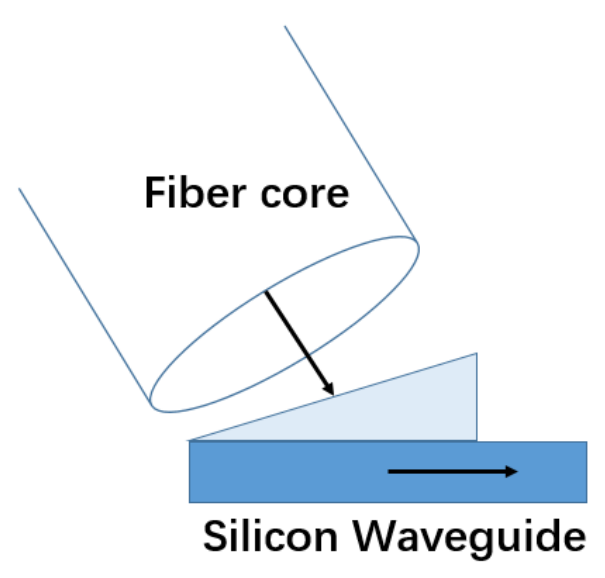

(c)

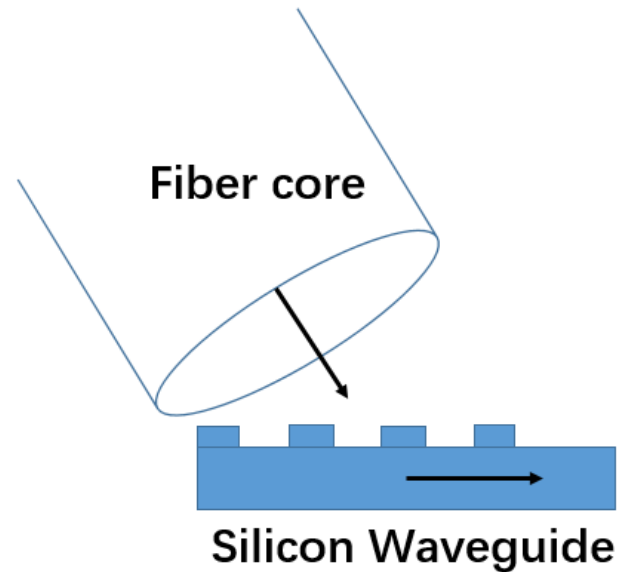

(d)

Figure 1.1 Schematics of (a) butt coupling, (b) end-fire coupling, (c) prism coupling and (d) grating coupling 
Unlike the edge coupling, which must couple light from the edge of the chip and requires post-processing, prism coupling and grating coupling can couple light to anywhere on the platform surface. The prism coupling has a prism with a refractive index larger than silicon and refracts light into the silicon waveguide. This technique comes with the risk of possibly damaging the silicon surface. The grating coupling has gratings that can be fabricated directly by etching. The etching step commonly requires a deep-UV fabrication process and can be done with the whole PICs design. [6]

Edge coupling has the advantages of high coupling efficiency, broadband operation, and polarization insensitivity. Experimentally, a $0.25 \mathrm{~dB}$ coupling loss has been achieved with a $300 \mathrm{~nm} \mathrm{3-dB}$ bandwidth for both TE and TM polarization at a $1550 \mathrm{~nm}$ center wavelength. Both prism coupling and grating coupling have the advantages of higher alignment tolerance and ease of testing.

\subsection{Grating coupler}

Grating couplers are the most commonly used component for coupling between the fiber and chip. Figure 1.6 shows a schematic of a 2D shallow-etched grating coupler.

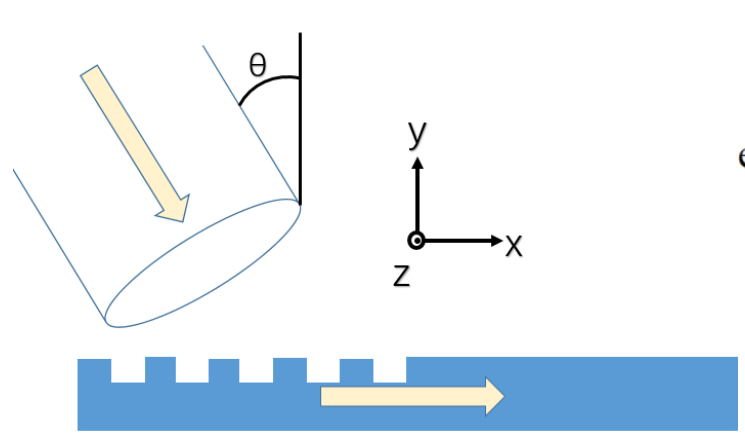

(a)

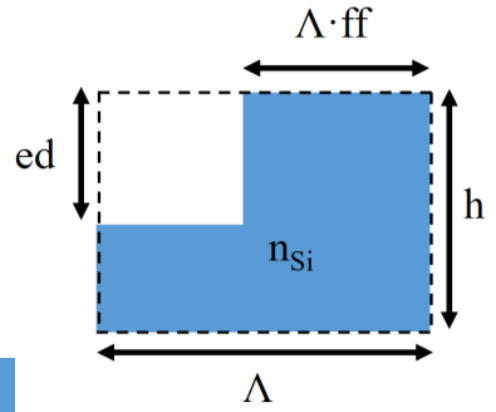

(b)

Figure 1.6 Schematics of (a) the shallow-etched grating coupler and (b) the side view of one period 
The periodic gratings of a grating coupler cause a periodic modulation of the effective index of the waveguide to satisfy the phase-matching condition and realize the coupling between fiber and chip. [6]

In the schematics of the grating coupler shown in Figure 1.6, $\Lambda$ is the period length of the gratings, $\mathrm{ff}$ is the fill-factor that is defined as the ratio of the filled length and period length, $h$ is the thickness of the waveguide and ed is the etched depth of the grating, and $\theta$ is the radiation angle.

There are two kinds of 2D grating coupler etching designs: a shallow-etched grating coupler and a fully-etched grating coupler. The etched depth ed is equal to the waveguide thickness $h$ of fully-etched grating couplers and less than the thickness of shallow-etched grating couplers. Some special designs such as apodizing [7-9], L-shape gratings [10], or inverted-T-shape gratings [11] were used to increase the performance of grating coupling. In 2-D grating coupler designs, more specialized structures such as square- $[12,13]$ or circular-shaped holes [14], fishbone-shaped gratings [15], and focusing gratings [16] have been proposed.

The incident angle of the fiber $\theta$ is generally slightly larger than 0 degrees to avoid a strong back reflection within the gratings [17]. However, vertical incident grating couplers were also demonstrated [18]. This particular design with $\theta=0$ could work as a bidirectional low-loss $3 \mathrm{~dB}$ power splitter.

In addition to fiber-to-chip coupling, grating couplers have been utilized as wavelength [19-23], polarization [24-27], or power splitters [25]. Table 1.1 lists some stateof-the-art, high-efficiency or broadband grating couplers that work for a single band . Those grating couplers have a similar or better coupling efficiency than the edge couplings 
did. However, the 3-dB bandwidth and the polarization insensitivity of grating coupling are still less than edge coupling. Although the bandwidth of the WDM system is $80 \mathrm{~nm}$, the demand for a higher data rate and faster network requires a better coupling design.

Table 1.1 State-of-the-art grating couplers work for a single wavelength.

\begin{tabular}{|c|c|c|c|c|c|}
\hline \multirow[t]{2}{*}{ REF } & \multicolumn{2}{|c|}{ COUPLING EFFICIENCY } & \multicolumn{2}{|c|}{ BANDWIDTH } & \multirow[t]{2}{*}{ PROCESS } \\
\hline & Simulated & Experimental & Simulated & Experimental & \\
\hline $\begin{array}{l}2015, \text { D. } \\
\text { BENEDIKOVIC } \\
{[28]}\end{array}$ & $85.7 \%$ & $85.3 \%$ & $3 \mathrm{~dB}: 60 \mathrm{~nm}$ & $3 \mathrm{~dB}: 60 \mathrm{~nm}$ & $\begin{array}{l}220 \mathrm{~nm} \text { SOI, } \\
\text { shallow etched }\end{array}$ \\
\hline 2015, L. YU [17] & $84 \%$ & $76 \%$ & $1 \mathrm{~dB}: 44 \mathrm{~nm}$ & $1 \mathrm{~dB}: 45 \mathrm{~nm}$ & $\begin{array}{l}220 \mathrm{~nm} \text { SOI, } \\
\text { shallow etched with } \\
\text { overlayer }\end{array}$ \\
\hline $\begin{array}{l}\text { 2017, R. } \\
\text { MARCHETTI, } \\
\text { [29] }\end{array}$ & $83 \%$ & $81 \%$ & $1 \mathrm{~dB}: 40 \mathrm{~nm}$ & $1 \mathrm{~dB}: 37.4 \mathrm{~nm}$ & $\begin{array}{l}260 \mathrm{~nm} \text { SOI, } \\
\text { shallow etched }\end{array}$ \\
\hline $\begin{array}{l}2019, \text { D. } \\
\text { BENEDIKOVIC } \\
{[10]}\end{array}$ & $95 \%$ & $91 \%$ & $1 \mathrm{~dB}: 40 \mathrm{~nm}$ & $3 \mathrm{~dB}: 40 \mathrm{~nm}$ & $\begin{array}{l}300 \mathrm{~nm} \text { SOI, } \\
\text { L-shape etched }\end{array}$ \\
\hline
\end{tabular}

\subsection{Polarization Independent Grating Couplers}

Due to the effective index contrast between the TE and TM polarization of a planar waveguide and the strong intrinsic dependency on the radiation angle of the gratings, conventional grating couplers have narrow spectral bandwidths and high polarization sensitivity.

A grating coupler that can split polarizations into different directions is easier to be realize than that can couple both TE and TM light into the same direction.

The very first two-dimensional grating coupler used as a polarization splitter was done by Taillaert et al. [30] in 2003. This design is shown in Figure 1.7 (a). It has a very narrow spectral bandwidth of $30 \mathrm{~nm}$ and a low coupling efficiency of $20 \%$. This architecture converts the two orthogonal modes, s- and p- polarization modes, of the fiber to TE mode into a grating coupler with two orthogonal output ports. Although this design 
is called a polarization independent grating coupler, it is a polarization splitting of the fiber modes only—not the planer waveguide modes.

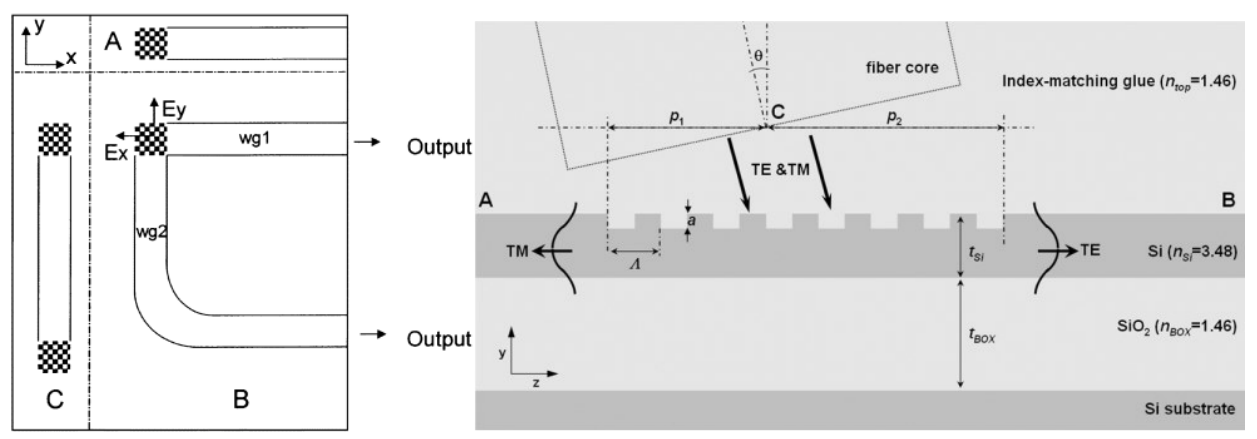

(a)

(b)

Figure 1.7 Design schematics of (a) the first 2D grating coupler used as a polarization splitter [30] (b) the first 1D polarization splitter grating coupler [26]

Tang et al. designed the first polarization splitting grating coupler in 2009 [26]. It coupled the TE polarization (known as s-polarization) to the forward direction and the TM polarization of fiber (known as p-polarization) to the backward direction. The schematic of this design is shown in Figure 1.7 (b). The 1-D structure is more useful and easier to fabricate compared with the design in [30]. This layout has a broad 3-dB bandwidth of 70 $\mathrm{nm}$ and a high coupling efficiency of $\sim 50 \%$.

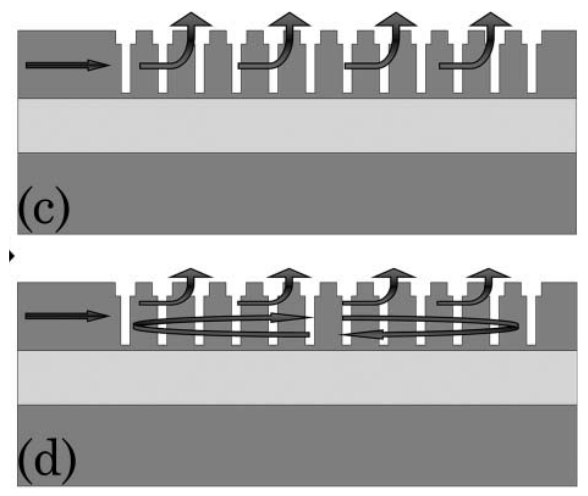

(a)

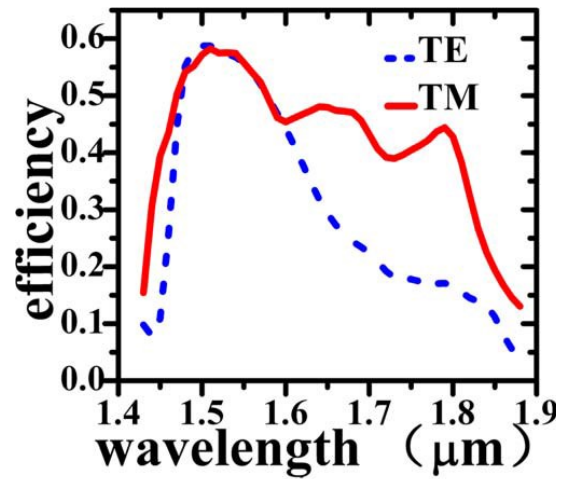

(b)

Figure 1.8 (a) Proposed architectures of the gratings and its working theory [11]. (b) The coupling efficiency spectrum 
Because of the strong polarization dependency of the grating coupler, it is almost impossible to realize polarization independence with a conventional grating 1D grating coupler. In Shao and Yang's work done in 2010 [11], they designed the first polarization 1D grating coupler. A special inverted T-shaped grating was used to create different effects for TE and TM mode. The simulation result showed that this design has a maximum coupling efficiency of around $58 \%$ and a $1-\mathrm{dB}$ bandwidth of $70 \mathrm{~nm}$. However, the inverted T-shape grating coupler is challenging to manufacture.

A reliable and fabricable 2-D polarization independent grating coupler has been designed with square-shaped gratings by Chen and Tsang in 2011 [31]. Figure 1.9 shows the special structure of this design. These square-shaped gratings identify the effective index of TE and TM polarization. This design has maximum coupling efficiencies of $64 \%$ and bandwidths of $65 \mathrm{~nm}$ for both polarizations. Although Chen and Tsang did not show the experimental work of this structure, square-shaped holes grating couplers have already been demonstrated in the early 2000 s.

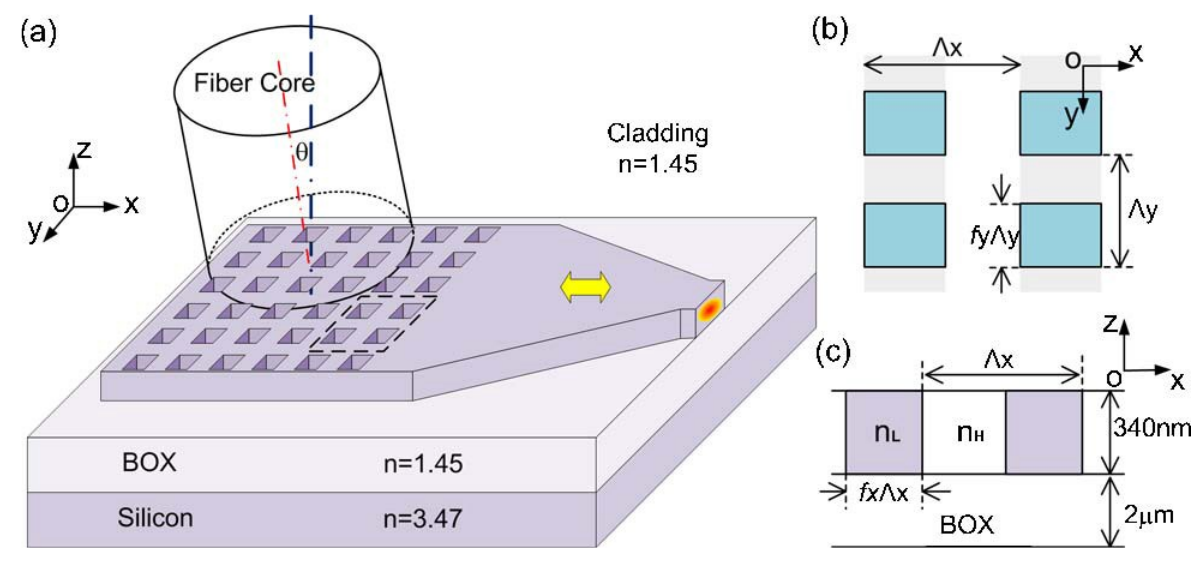

Figure 1.9 Proposed architectures of 2D polarization independent grating coupler [31]

Some novel attempts such as the two-layer grating coupler done by Feng and Zhou in 2007 and the non-uniform grating coupler proposed by Song et al. in 2015 [32] can also 
realize polarization independence. Table 1.2 listed some state-of-the-art polarization independent grating couplers.

Table 1.2 State-of-the-art polarization independent grating couplers

\begin{tabular}{|c|c|c|c|c|c|}
\hline REF & \multicolumn{2}{|c|}{ COUPLING EFFICIENCY } & \multicolumn{2}{|c|}{ BANDWIDTH } & PROCESS \\
\hline & Simulated & Experimental & Simulated & Experimental & \\
\hline $\begin{array}{l}\text { 2015, } \\
\text { ZHANG } \\
{[33]}\end{array}$ & $\begin{array}{l}69.69 \%(\mathrm{TE}) \\
75.15 \%(\mathrm{TM})\end{array}$ & $\begin{array}{l}\text { Simulation } \\
\text { only }\end{array}$ & $\begin{array}{l}1 \mathrm{~dB}: \\
30 \mathrm{~nm}(\mathrm{TE}), \\
40 \mathrm{~nm}(\mathrm{TM})\end{array}$ & $\begin{array}{l}1 \mathrm{~dB}: \\
90 \mathrm{~nm}\end{array}$ & $\begin{array}{l}460 \mathrm{~nm} \text { SOI, } \\
\text { Shallow etched }\end{array}$ \\
\hline $\begin{array}{l}\text { 2009, } \\
\text { TANG [26] }\end{array}$ & $-3 \mathrm{~dB}$ & $\begin{array}{l}\text { Simulation } \\
\text { only }\end{array}$ & $\begin{array}{l}3 \mathrm{~dB}: \\
70 \mathrm{~nm}(\mathrm{TE}) \\
70 \mathrm{~nm} \text { (TM) }\end{array}$ & $\begin{array}{l}\text { Simulation } \\
\text { only }\end{array}$ & $\begin{array}{l}260 \mathrm{~nm} \text { SOI, } \\
\text { Shallow etched }\end{array}$ \\
\hline $\begin{array}{l}\text { 2014, } \\
\text { CHENG } \\
{[34]}\end{array}$ & $\begin{array}{l}-3.3 \mathrm{~dB}(\mathrm{TE}) \\
-2.2 \mathrm{~dB}(\mathrm{TM})\end{array}$ & $\begin{array}{l}-4.3 \mathrm{~dB}(\mathrm{TE}) \\
-3.2 \mathrm{~dB}(\mathrm{TM})\end{array}$ & $\begin{array}{l}1 \mathrm{~dB}: \\
60 \mathrm{~nm}(\mathrm{TE}), \\
30 \mathrm{~nm} \text { (TM) }\end{array}$ & $\begin{array}{l}1 \mathrm{~dB}: \\
58 \mathrm{~nm}(\mathrm{TE}), \\
28 \mathrm{~nm}(\mathrm{TM})\end{array}$ & $\begin{array}{l}\text { 340nm SOI, } \\
\text { fully etched }\end{array}$ \\
\hline $\begin{array}{l}2015, \\
\text { SONG [32] }\end{array}$ & $\begin{array}{l}-6.9 \mathrm{~dB}(\mathrm{TE}) \\
-7.1 \mathrm{~dB}(\mathrm{TM})\end{array}$ & $\begin{array}{l}-7.8 \mathrm{~dB}(\mathrm{TE}) \\
-8.0 \mathrm{~dB}(\mathrm{TM})\end{array}$ & $\begin{array}{l}1 \mathrm{~dB}: \\
40 \mathrm{~nm}(\mathrm{TE}), \\
40 \mathrm{~nm}(\mathrm{TM})\end{array}$ & Not given & $\begin{array}{l}220 \mathrm{~nm} \text { SOI, } \\
\text { Shallow etched }\end{array}$ \\
\hline
\end{tabular}

\subsection{Dual-wavelength Grating Couplers}

In the WDM system, $1310 \mathrm{~nm}$ and $1550 \mathrm{~nm}$ are the two most used wavelengths of light because they have a low loss in the optical communication system. Those two wavelength bands are also known as $\mathrm{O}$ band and $\mathrm{C}$ band.

With increasing demand for wavelength-division-multiplexing (WDM) systems in fiber-to-the-home network (FTTH) services, low-cost dual-band (O- and C-bands) on-chip transceivers are required. In the traditional optical-electrical-optical (O-E-O) transponder, $1310 \mathrm{~nm}$ and $1550 \mathrm{~nm}$ signals are converted to an electronic signal and converted back to the optical signal again. While O-E-O transponders are slow and low-efficiency, all-optical transponders have a smaller footprint, low power cost, and fast transfer speed. A dual-band grating coupler can be used as a transceiver in the system.

Similar to the polarization independent grating coupler, a wavelength splitting grating coupler with the same polarization, or a dual-wavelength grating coupler with different polarizations, are easier to achieve. Figure 1.10 shows those two kinds of designs. 


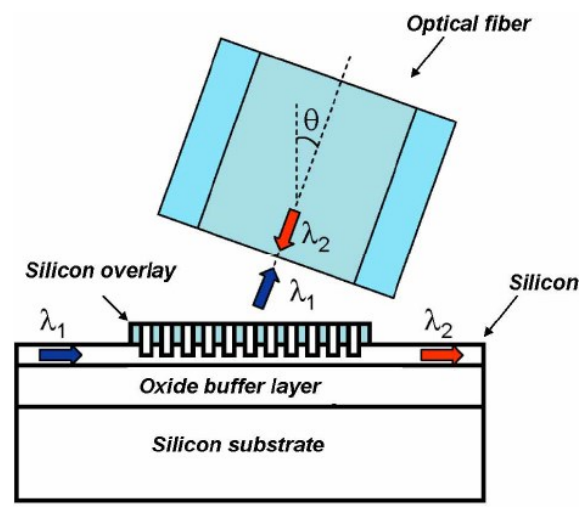

(a)

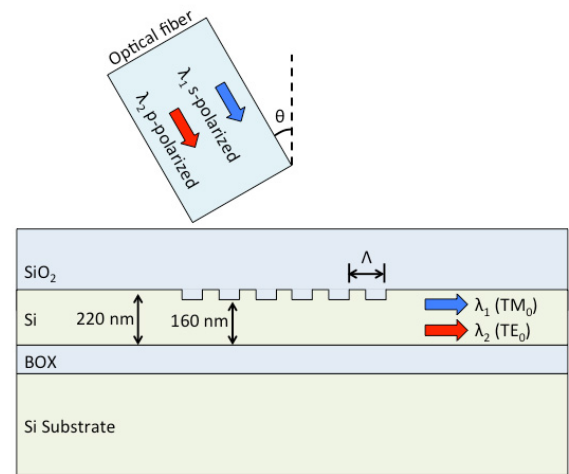

(b)

Figure 1.10 Design schematics of (a) the wavelength splitting grating coupler with the same polarization [23] and (b) a dual-wavelength grating coupler with different polarizations [21]

The wavelength splitting grating coupler (figure 1.10 (a) [23]) is similar to the polarization splitting grating coupler (figure 1.7 (b)). This design can give high coupling efficiencies of $55 \%$ and bandwidth of $50 \mathrm{~nm}$ for both wavelengths. The simple fabrication steps make this design useful. The dual-wavelength grating coupler with different polarizations design done by Streshinsky et al. in 2013 [21] has a low transmission of 20\% for $1550 \mathrm{~nm}$ and $\sim 15 \%$ for $1310 \mathrm{~nm}$ and 3 -dB bandwidths of $60 \mathrm{~nm}$ for both wavelengths. Although this design is low efficiency, it can apply the same method as [18] did and work as a polarization splitter for fiber modes.

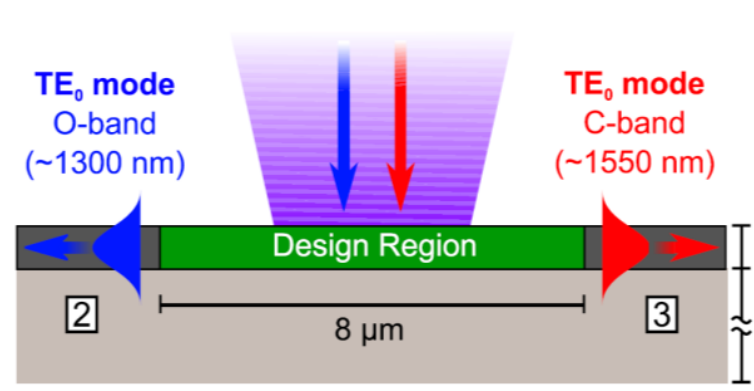

(a)

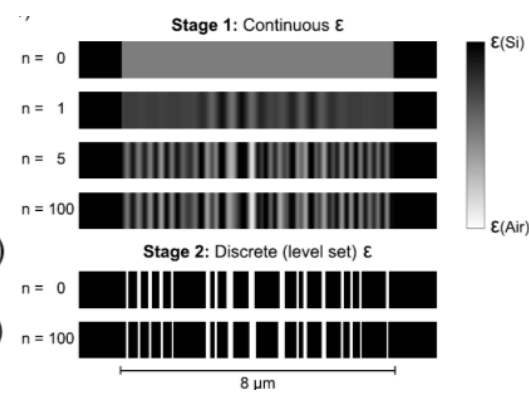

(b)

Figure 1.11 Design schematics of (a) the vertical incident wavelength splitting grating coupler done with inverse design and (b) the optimization with the different iteration number [22] 
There's almost no better 1-D wavelength splitting design than [21]. However, researchers were trying to design vertical incident grating couplers that can package more easily. Because the periodic vertical incident grating coupler has two opposite direction ports with identical outputs, it is impossible to develop a vertical incident wavelength splitting grating coupler. Vučković et al. presented a non-uniform vertical incident grating coupler by using inverse design in 2014 (Figure 1.11) [22]. This novel design has a 30\% coupling efficiency and a 3-dB bandwidth of $\sim 40 \mathrm{~nm}$. Although these performances are not good enough, the inverse design shows a fast way to do the non-uniform grating coupler design. In 2018, the same group presented a gradient-based algorithm to design fullyautomated optimized grating couplers [24]. By using a better algorithm of machine learning, the group was able to attain the optimized result shown in Figure 1.12 (a). This layout has coupling efficiencies of $69 \%, 3-\mathrm{dB}$ bandwidths of $40 \mathrm{~nm}$, and minimum feature size of 50 $\mathrm{nm}$. These results are much better than their previous design but hard to fabricate.

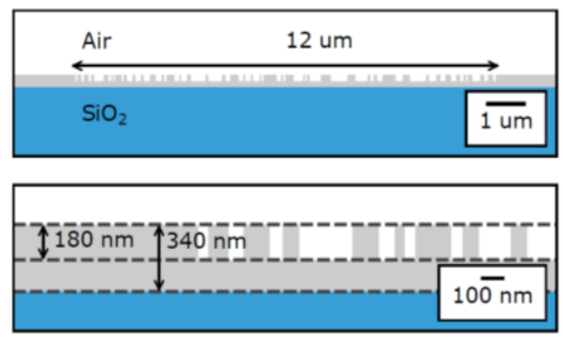

(a)
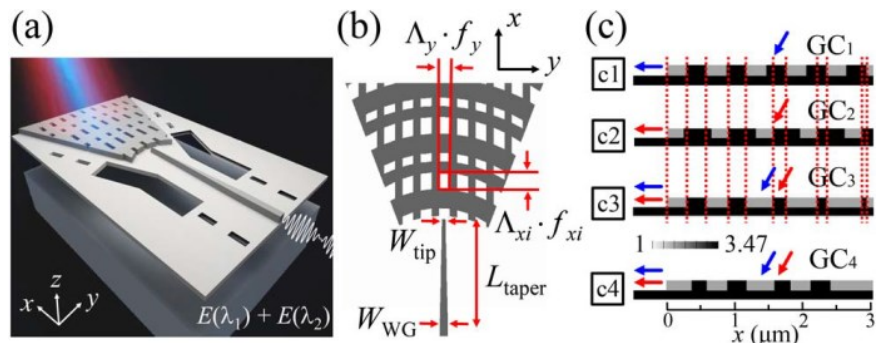

(b)

Figure 1.12 Design schematics of (a) the vertical incident wavelength splitting grating coupler done with inverse design [24] and (b) a dual-wavelength grating coupler made with a gradient-based algorithm [35]

Zhou et al. showed a tailorable dual-wavelength-band focusing subwavelength grating coupler in 2018 [35]. It can couple both $1480 \mathrm{~nm}$ and $1660 \mathrm{~nm}$ or $1600 \mathrm{~nm}$ light to TE polarization in the same direction waveguide with a coupling efficiency around $45 \%$ 
and bandwidths around $40 \mathrm{~nm}$. It shows the possibility of designing a single direction $\mathrm{O}-$ and C-band grating coupler.

Other than using silicon, silicon nitride $\left(\mathrm{Si}_{3} \mathrm{~N}_{4}\right)$ was also used as the waveguide material [36]. $\mathrm{Si}_{3} \mathrm{~N}_{4}$ has a lower reflective index $\left(\mathrm{n}_{\mathrm{Si} 3 \mathrm{~N} 4}\right.$ is around 2 for $1310 \mathrm{~nm}$ and 1550 $\mathrm{nm}$ ) and loss than Si. It can identify the average effective index of the mode with different wavelengths comfortably. In this design, the conventional uniform grating coupler has a coupling efficiency of $35 \%$ and a bandwidth of $80 \mathrm{~nm}$.

Table 1.3 shows all the state-of-the-art dual-band grating couplers

Table 1.3 State-of-the-art dual-wavelength grating couplers

\begin{tabular}{|c|c|c|c|c|c|c|}
\hline \multirow[t]{2}{*}{ REF } & \multirow[t]{2}{*}{$\begin{array}{l}\text { OPERATION } \\
\text { WAVELENGTH } \\
\text { AND } \\
\text { POLARIZATIO } \\
\text { N }\end{array}$} & COUPLING E & FICIENCY & BANDWID? & & PROCESS \\
\hline & & Simulated & $\begin{array}{l}\text { Experimenta } \\
1\end{array}$ & Simulated & $\begin{array}{l}\text { Experimenta } \\
1\end{array}$ & \\
\hline $\begin{array}{l}\text { 2013, M. } \\
\text { STRESHIN } \\
\text { SKY [21] }\end{array}$ & $\begin{array}{l}1290 \mathrm{~nm}(\mathrm{TM}) \& \\
1570 \mathrm{~nm}(\mathrm{TE})\end{array}$ & $\begin{array}{l}17 \%(1290 \\
\mathrm{nm}) \& \\
21 \%(1570 \\
\mathrm{nm})\end{array}$ & $\begin{array}{l}-8.2 \mathrm{~dB} \\
(1296 \mathrm{~nm}) \\
\&-7.1 \mathrm{~dB} \\
(1576 \mathrm{~nm})\end{array}$ & $\begin{array}{l}3 \mathrm{~dB}: 50 \\
\mathrm{~nm}(1290 \\
\mathrm{nm} \& 1570 \\
\mathrm{~nm})\end{array}$ & $\begin{array}{l}1.5 \mathrm{~dB}: 18 \\
\mathrm{~nm}(1296 \\
\mathrm{nm}) \& 35 \\
\mathrm{~dB}(1576 \\
\mathrm{nm})\end{array}$ & $\begin{array}{l}220 \mathrm{~nm} \text { SOI, } \\
\text { shallow } \\
\text { etched }\end{array}$ \\
\hline $\begin{array}{l}\text { 2014, A. Y. } \\
\text { PIGGOTT } \\
{[22]}\end{array}$ & $\begin{array}{l}1300 \mathrm{~nm}(\mathrm{TE}) \& \\
1550 \mathrm{~nm}(\mathrm{TE})\end{array}$ & $\begin{array}{l}29.37 \%(1293 \\
\mathrm{nm}) \& \\
45.44 \%(1540 \\
\mathrm{nm})\end{array}$ & Not given & $\begin{array}{l}3 \mathrm{~dB}: \text { Not } \\
\text { given, but } \\
\text { close to } 30 \\
\mathrm{~nm} \text { from } \\
\text { plots } \\
\text { reading }\end{array}$ & Not given & $\begin{array}{l}220 \mathrm{~nm} \text { SOI, } \\
\text { fully etched, } \\
\text { inverse } \\
\text { design, } \\
\text { vertical } \\
\text { incident }\end{array}$ \\
\hline $\begin{array}{l}\text { 2018, L. SU } \\
{[24]}\end{array}$ & $\begin{array}{l}1310 \mathrm{~nm}(\mathrm{TE}) \& \\
1490 \mathrm{~nm}(\mathrm{TE})\end{array}$ & $\begin{array}{l}1.5 \mathrm{~dB}(1310 \\
\mathrm{nm}) \& \\
1.6 \mathrm{~dB}(1490 \\
\mathrm{nm})\end{array}$ & $\begin{array}{l}\text { Simulation } \\
\text { only }\end{array}$ & $\begin{array}{l}1 \mathrm{~dB}: 20 \\
\mathrm{~nm} \\
(1310 \mathrm{~nm}) \\
\& 24 \mathrm{~dB} \\
(1490 \mathrm{~nm})\end{array}$ & $\begin{array}{l}\text { Simulation } \\
\text { only }\end{array}$ & $\begin{array}{l}340 \mathrm{~nm} \text { SOI, } \\
\text { shallow } \\
\text { etched, } \\
\text { gradient-based } \\
\text { algorithm, } \\
\text { vertical } \\
\text { incident }\end{array}$ \\
\hline $\begin{array}{l}\text { 2018, W. } \\
\text { ZHOU [35] }\end{array}$ & $\begin{array}{l}\text { A. } 1480 \mathrm{~nm} \text { (TE) } \\
\& 1600 \mathrm{~nm} \text { (TE) } \\
\text { B. } 1480 \mathrm{~nm} \text { (TE) } \\
\& 1660 \mathrm{~nm} \text { (TE) }\end{array}$ & $\begin{array}{l}\text { A. } 47.3 \% \\
(1480 \mathrm{~nm}) \& \\
42.9 \%(1600 \\
\mathrm{nm}) \\
\text { B. } 45.7 \% \\
(1480 \mathrm{~nm}) \& \\
45.3 \%(1660 \\
\mathrm{nm})\end{array}$ & $\begin{array}{l}\text { A. } 18.3 \% \\
(1486 \mathrm{~nm}) \\
\& 20.1 \% \\
(1594.5 \mathrm{~nm}) \\
\text { B. } 14.5 \% \\
(1481.5 \mathrm{~nm}) \\
\& 17.5 \% \\
(1661.5 \mathrm{~nm})\end{array}$ & $\begin{array}{l}\text { Not given, } \\
\text { but close to } \\
\text { the } \\
\text { experimenta } \\
1 \text { result from } \\
\text { plots } \\
\text { reading }\end{array}$ & $\begin{array}{l}3 \mathrm{~dB}: \text { A. } 55 \\
\mathrm{~nm}(1486 \\
\mathrm{nm}) \& 30.5 \\
\mathrm{~nm}(1594.5 \\
\mathrm{nm}) \text { B. } 44 \\
\mathrm{~nm}(1481.5 \\
\mathrm{nm}) \& 39.5 \\
\mathrm{~nm}(1661.5 \\
\mathrm{nm})\end{array}$ & $\begin{array}{l}340 \mathrm{~nm} \text { SOI, } \\
\text { shallow } \\
\text { etched, } \\
\text { focusing, } \\
\text { inverse taper, } \\
\text { suspended- } \\
\text { membrane } \\
\text { waveguide }\end{array}$ \\
\hline $\begin{array}{l}\text { 2019, W. } \\
\text { ZHOU [37] }\end{array}$ & $\begin{array}{l}\text { A. } 1560 \mathrm{~nm} \text { (TE) } \\
\& 2255 \mathrm{~nm} \text { (TE) } \\
\text { B. } 1487 \mathrm{~nm} \text { (TE) } \\
\& 2331 \mathrm{~nm} \text { (TE) }\end{array}$ & $\begin{array}{l}\text { A. } 60.1 \% \\
(1550 \mathrm{~nm}) \& \\
60 \%(2244 \\
\mathrm{nm})\end{array}$ & $\begin{array}{l}\text { A. } 20.2 \% \\
(1560 \mathrm{~nm}) \\
\& 25.8 \% \\
(2255 \mathrm{~nm}) \\
\text { B. } 20.6 \%\end{array}$ & $\begin{array}{l}\text { Not given, } \\
\text { but close to } \\
\text { the } \\
\text { experimenta } \\
1 \text { result from }\end{array}$ & $\begin{array}{l}1 \mathrm{~dB}: 38 \mathrm{~nm} \\
\& 54 \mathrm{~nm}\end{array}$ & $\begin{array}{l}500 \mathrm{~nm} \text { SOI, } \\
\text { shallow } \\
\text { etched, } \\
\text { focusing, } \\
\text { inverse taper, }\end{array}$ \\
\hline
\end{tabular}




\begin{tabular}{|c|c|c|c|c|c|c|}
\hline & & $\begin{array}{l}\text { B. } 61.6 \% \\
(1545 \mathrm{~nm}) \& \\
60 \%(2243 \\
\mathrm{nm})\end{array}$ & $\begin{array}{l}(1487 \mathrm{~nm}) \\
\& 26.9 \% \\
(2331 \mathrm{~nm})\end{array}$ & $\begin{array}{l}\text { plots } \\
\text { reading }\end{array}$ & & $\begin{array}{l}\text { suspended- } \\
\text { membrane } \\
\text { waveguide }\end{array}$ \\
\hline $\begin{array}{l}\text { 2018, S. } \\
\text { NAMBIAR } \\
{[36]}\end{array}$ & $\begin{array}{l}1310 \mathrm{~nm} \text { (TE) \& } \\
1550 \mathrm{~nm} \text { (TE) }\end{array}$ & $\begin{array}{l}34 \%(1562 \\
\mathrm{nm}) \& 40 \% \\
(1290 \mathrm{~nm}) \\
\text { With a } \\
\text { distributed } \\
\text { Bragg } \\
\text { reflector: } 60 \% \\
\text { for both } \lambda\end{array}$ & $\begin{array}{l}-7.9 \mathrm{~dB} \\
(1289 \mathrm{~nm}) \\
\& \\
-8.2 \mathrm{~dB} \\
(1551 \mathrm{~nm})\end{array}$ & $\begin{array}{l}1 \mathrm{~dB}: 49 \\
\mathrm{~nm} \\
(1289 \mathrm{~nm}) \\
\& 47 \mathrm{~nm} \\
(1551 \mathrm{~nm}) \\
\text { With a } \\
\text { distributed } \\
\text { Bragg } \\
\text { reflector: } 50 \\
\text { nm for both } \\
\lambda\end{array}$ & $\begin{array}{l}1 \mathrm{~dB}: 34 \\
\mathrm{~nm} \\
(1289 \mathrm{~nm}) \& \\
48 \mathrm{~nm}(1551 \\
\mathrm{nm})\end{array}$ & $\begin{array}{l}700 \mathrm{~nm} \mathrm{SiN} \text {, } \\
\text { shallow } \\
\text { etched, }\end{array}$ \\
\hline $\begin{array}{l}\text { 2019, L. } \\
\text { CHENG } \\
{[19]}\end{array}$ & $\begin{array}{l}1310 \mathrm{~nm} \text { (TE) \& } \\
1550 \mathrm{~nm} \text { (TE) }\end{array}$ & $\begin{array}{l}24.6 \%(1325 \\
\mathrm{nm}) \& 19.3 \% \\
(1533 \mathrm{~nm}) \\
\text { With bottom } \\
\text { mirror: } 70.8 \% \\
(1321 \mathrm{~nm}) \& \\
45 \%(1543 \\
\mathrm{nm})\end{array}$ & $\begin{array}{l}\text { Simulation } \\
\text { only }\end{array}$ & $\begin{array}{l}3 \mathrm{~dB} \text { : Not } \\
\text { given, but } \\
\text { close to } 50 \\
\mathrm{~nm} \text { from } \\
\text { plots } \\
\text { reading }\end{array}$ & $\begin{array}{l}\text { Simulation } \\
\text { only }\end{array}$ & $\begin{array}{l}220 \mathrm{~nm} \text { SOI, } \\
\text { fully etched, } \\
\text { vertical } \\
\text { incident }\end{array}$ \\
\hline $\begin{array}{l}\text { 201, J. } \\
\text { TAN [20] }\end{array}$ & $\begin{array}{l}1310 \mathrm{~nm} \text { (TE) \& } \\
1490 \mathrm{~nm} \text { (TE) }\end{array}$ & $\begin{array}{l}10 \%(1320 \\
\mathrm{nm}) \& \\
14 \%(1510 \\
\mathrm{nm}) \\
\\
\text { With } \mathrm{Au} \\
\text { substrate } 41 \% \\
(1465 \mathrm{~nm}) \& \\
24 \%(1585 \\
\mathrm{nm})\end{array}$ & $\begin{array}{l}\text { Simulation } \\
\text { only }\end{array}$ & Not given & $\begin{array}{l}\text { Simulation } \\
\text { only }\end{array}$ & $\begin{array}{l}\text { SOI with } \\
\text { silicon } \\
\text { overlay, } \mathrm{Au} \\
\text { substrate }\end{array}$ \\
\hline $\begin{array}{l}\text { 2007, G. } \\
\text { ROELKEN } \\
\text { S, [23] }\end{array}$ & $\begin{array}{l}1310 \mathrm{~nm} \text { (TE) \& } \\
1490 \mathrm{~nm}(\mathrm{TE})\end{array}$ & $\begin{array}{l}55 \% \text { for both } \\
\lambda\end{array}$ & $\begin{array}{l}\text { Simulation } \\
\text { only }\end{array}$ & $\begin{array}{l}3 \mathrm{~dB}: 55 \\
\mathrm{~nm} \\
(1310 \mathrm{~nm}) \\
\& 60 \mathrm{~dB} \\
(1490 \mathrm{~nm})\end{array}$ & $\begin{array}{l}\text { Simulation } \\
\text { only }\end{array}$ & $\begin{array}{l}220 \mathrm{~nm} \text { SOI } \\
\text { with silicon } \\
\text { overlay, } \\
\text { Demultiplexin } \\
\mathrm{g}\end{array}$ \\
\hline
\end{tabular}

\subsection{Thesis Objectives and Organization}

Most of dual-wavelength grating couplers in table 1.3 required complex structures and did not have center wavelengths at $1310 \mathrm{~nm}$ and $1550 \mathrm{~nm}$. Additional bottom reflectors were also applied to increase coupling efficiencies. Importantly, all the dual-wavelength grating couplers can only operate with a single polarization. The goal of this thesis is to study and optimize a dual-wavelength grating coupler with polarization insensitivity and high efficiency. 
Chapter 2 reviews the physics theory of grating couplers. Bragg condition provides an approximate analyzing method of the periodical grating structure. Different effective index methods will be introduced to calculate the approximate effective index. Both the Bragg condition and effective index approximation are used to determine the parameters of the grating structure. Finite Difference Time Domain Method helps analyze performances of grating coupler designs and will be presented in chapter 2 as well.

In chapter 3, a simple vertical incident dual-band grating coupler structure will be shown. While a conventional grating coupler design flow will be presented in chapter 3 , a polarization independent grating coupler design will be introduced in chapter 4 . Chapter 4 will also show a polarization splitting grating coupler that can support both $1310 \mathrm{~nm}$ and $1550 \mathrm{~nm}$ light. Common optimized progress will be explained in chapter 3 as well.

Chapter 5 outlines the effective index analysis method for a subwavelength grating structure with both O-band and C-band and both TE and TM polarizations at the same time. Dual-band polarization independent designs with square-shaped holes and subwavelength slits or shallow etched gratings will be present. Tolerance analysis for the fabricable dualband polarization independent grating coupler is shown as well.

Finally, chapter 6 concludes all the works and achievements of this thesis and outlines ideas for future progress in polarization and wavelength insensitive grating couplers. 


\section{Chapter 2:}

\section{Grating Theory and Numerical Methods}

In this chapter, fundamental theories of the grating coupler and simulating numerical methods will be presented. Bragg's law and Bragg condition are introduced at the beginning. They are the simplest and most fundamental theory to analyze periodic structures. The figure of Merit and some other coupler performance analysis will be exhibited as well. Afterward, effective index approximations will be presented. These methods are used to obtain an approach value of the effective index of mode propagating in a slab waveguide. At the end of this chapter, the numerical simulation method FiniteDifference Time-Domain (FDTD) method is introduced.

\subsection{Bragg Condition}

\subsubsection{Bragg's law}

There are two basic methods-Floquet or Bloch theorem [38, 39] and the perturbation method $[40,41]$ — for analyzing the periodic guided-wave grating problems. While the Floquet or Bloch theorem analysis is exact but complicated, the perturbation method is a more straightforward analysis method that can be used to describe the effects of gratings on the interacting modes using two coupled-mode equations. 
Braggs' Law can be a simple graphic representation of the perturbation method. It gives the angles for coherent and incoherent scattering from a crystal lattice. Figure 2.1 shows a schematic of the Bragg diffraction.

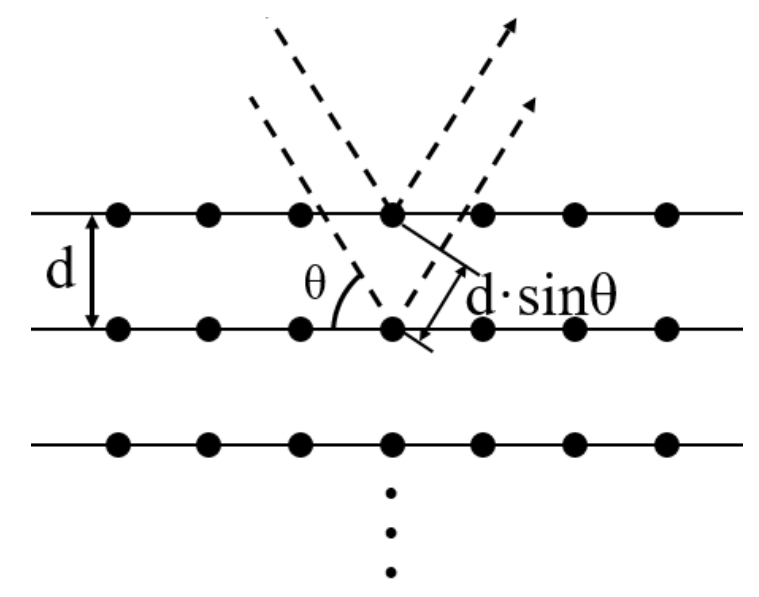

Figure 2.1 Schematic of the Bragg diffraction

Where $d$ is the interplanar distance of a crystalline solid, $\theta$ is the scattering angle.

This schematic shows a plane wave (shown as two beams with identical wavelength $\lambda$ and phase in this example to simplify the explanation) scattered from the lattice planes. The wave scattered from the second interplanar will travel an extra length of $2 d \sin \theta$ than the wave scattered from the first interplanar. These scattered waves will be in phase when they interfere constructively, which means the path length difference between the waves will be equal to an integer multiple of the wavelength. So it will have:

$$
2 d \sin \theta=m \lambda
$$

where $m$ is a positive integer. For a periodic structure such as gratings, the $d$ can be written as $\Lambda$, which is the period length of the periodic structure, and $\theta$ will be understood as the incident angle. If the refractive index is considered, equation 2.1 can be rewritten as:

$$
2 \Lambda \sin \theta=m \frac{\lambda_{0}}{n}
$$


where $n$ is the effective refractive index (also known as the average refractive index) of the gratings, $\lambda_{0}$ is the wavelength of the plane wave in free space. Equation 2.1 and 2.2 are the Bragg's law equation.

\subsubsection{Bragg condition}

The gratings of a grating coupler can be replaced by a structure with a crystalline solid, as Figure 2.2 shows.

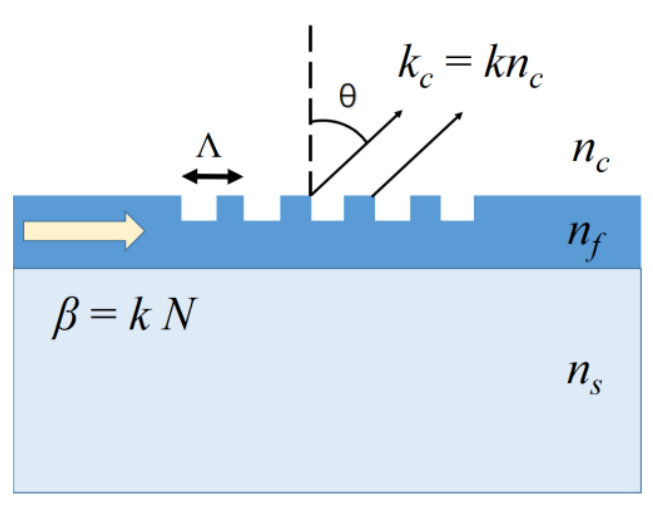

(a)

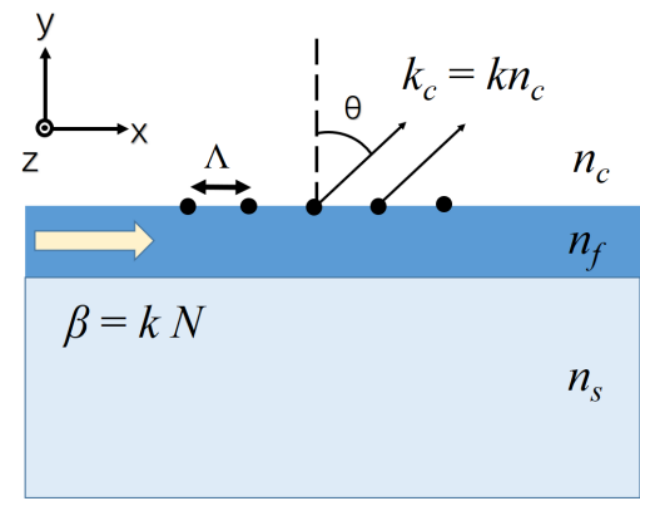

(b)

Figure 2.2 Schematics of (a) a one-dimensional grating coupler and (b) a crystalline solid approach grating coupler

A guided wave that is propagating in a slab waveguide with a series of gratings will diffract because of the small perturbation of the waveguide. For a single grating element (perturbation), the wave scattered is weak. However, the wave scattered by all gratings superimposes constructively in the same direction and the total diffracted wave will have a peak in this direction. Bragg's law (equation 2.2) can be applied and rewritten to simplify the grating structure analysis [6]. A constructive interference for a grating structure occurs while

$$
\beta-k_{c} \sin \theta=m K
$$


where $\beta$ represents the propagation constant of the wave propagating in the waveguide, $k_{c}$ is the propagation constant of the scattered wave propagating in the cladding of the waveguide, $K$ is the grating wave vector, they can be written as:

$$
\begin{gathered}
\beta=\frac{2 \pi n_{\mathrm{eff}}}{\lambda_{0}} \\
k_{c}=\frac{2 \pi n_{c}}{\lambda_{0}} \\
K=\frac{2 \pi}{\Lambda}
\end{gathered}
$$

where $n_{\text {eff }}$ donates the average effective index of the transmit mode propagating in the grating waveguide, $n_{c}$ represents the refractive index of the cladding of the grating coupler.

From every occurred constructive interference, the scattered wave in the grating coupler that diffracts to a direction can be observed. In equation $2.3, m$ represents the constructive interference result and refers to the $m$-th order Bragg diffraction.

Equation 2.3 can be represented by equation $2.4,2.5$, and 2.6 as:

$$
\frac{2 \pi n_{\mathrm{eff}}}{\lambda_{0}}-\frac{2 \pi n_{c}}{\lambda_{0}} \sin \theta=m \frac{2 \pi}{\Lambda}
$$

By simplifying, it can be rewritten as:

$$
n_{\text {eff }}-n_{c} \sin \theta=m \frac{\lambda_{0}}{\Lambda}
$$

Equation 2.8 is the Bragg condition for the grating coupler. Bragg condition is an approach for grating design and only exact for infinite gratings.

Notably, $\beta, k_{c}$ and $K$ are constant for a known grating structure. This means there will be a limited amount of radiated angle $\theta$ that can satisfy the equation. A longer period length will lead to a shorter grating wave vector and more diffraction orders. 
Figure 2.3 (a) and (b) show the Bragg condition schematically with first-order (where $\mathrm{m}=1$ ) and second-order (where $\mathrm{m}=2$ ).

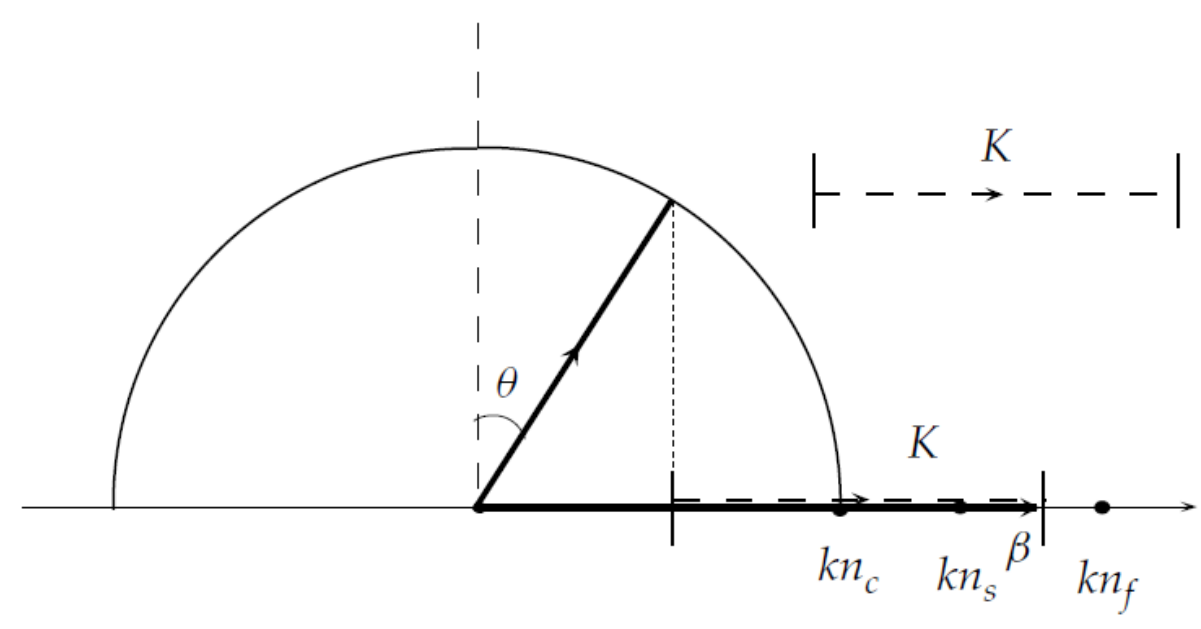

(a)

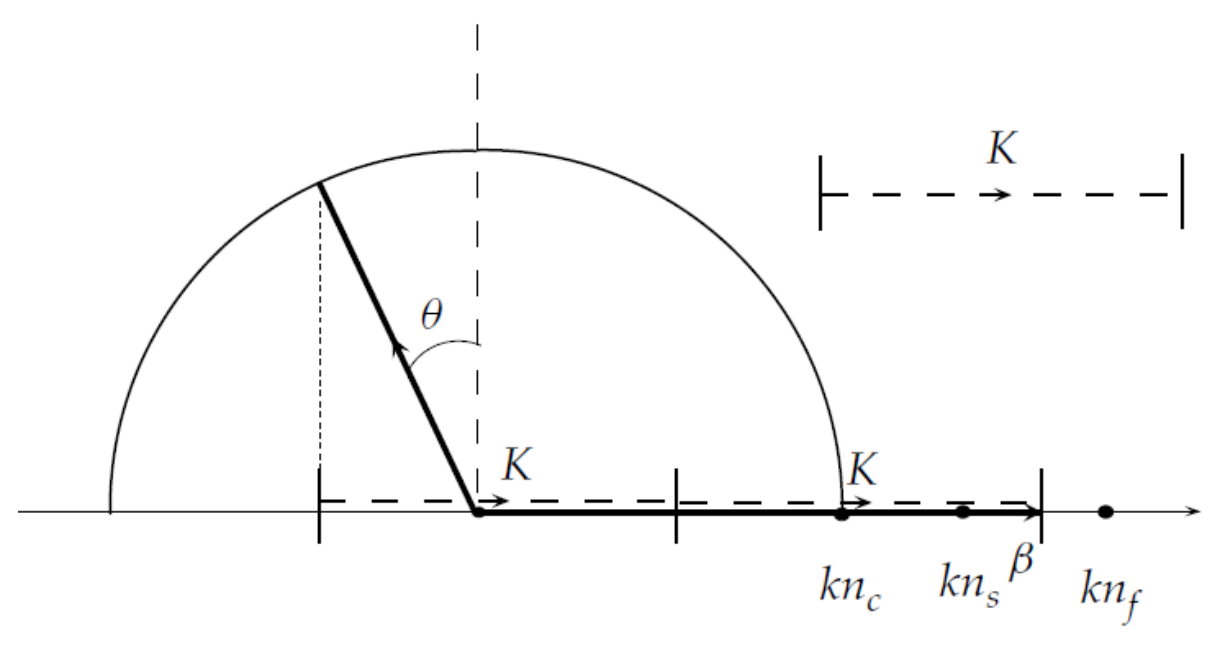

(b)

Figure 2.3 Schematic Bragg condition of (a) the first-order and (b) the second-order [55]

In figure 2.3, $\beta, k_{c}$ and $K$ are constant. The semi-circle is the wave vector of the cladding layer with a radiation angle $\theta$. A higher diffraction order leads to a smaller $\theta$ (in the figure, $\theta$ is decreased from a positive angle to a negative angle). There will be no thirdorder diffraction in this example because $3 K$ is larger than $2 k_{\text {c }}$. 


\subsection{Analysis of the Grating Performances}

\subsubsection{Figure of Merit}

The Figure of Merit (FoM) is used to compare the performances of the grating couplers. It includes:

1. Coupling Efficiency (CE) / Coupling Loss: the ratio between the power of the mode ( $\left.\mathrm{P}_{\text {coupled }}\right)$ coupled into or out from the waveguide and the total input power from the fiber or waveguide $\left(\mathrm{P}_{\text {total }}\right)$. CE and coupling loss are also known as transmission. $\mathrm{CE}$ is usually expressed in percentage $(\%)$ or decibels $(\mathrm{dB})$ as $10 \log _{10}\left(\mathrm{P}_{\text {coupled }} / \mathrm{P}_{\text {total }}\right)$.

2. 3-dB and 1-dB bandwidth: the bandwidth (spectrum wavelength range) of the coupled light that has a coupling efficiency greater than half $(3-\mathrm{dB})$ or $80 \%(1-\mathrm{dB})$ of the maximum coupling efficiency.

3. Back reflection: the ratio between the power of light propagating in the waveguide with the designed direction $\left(\mathrm{P}_{\text {coupled }}\right)$ and the power of the light propagated in the opposite direction's waveguide $\left(\mathrm{P}_{\text {backreflection }}\right)$. It is expressed as $\mathrm{P}_{\text {backreflection }} / \mathrm{P}_{\text {coupled. }}$

For any application, a high coupling efficiency (low-loss) grating coupler is required. In the WDM system, the bandwidth of a grating coupler decided the maximum data rate or channel numbers of the system. The back reflection is usually avoided by setting a small incident angle. However, in some sensing applications, a strong back reflection is required.

A grating coupler usually has a lower coupling efficiency and a narrower bandwidth compared with an edge coupler. The strong wavelength and polarization sensitivity of the grating coupler is one of the most important issues under research. 
A fundamental mode propagating in the waveguide has a Gaussian-like field distribution. When the light couples out from the chip to fiber, most power of the light coupled out from the grating coupler will be caused the by first few gratings. This issue is shown in figure 2.4 [7] and known as a mode mismatch of the gratings.

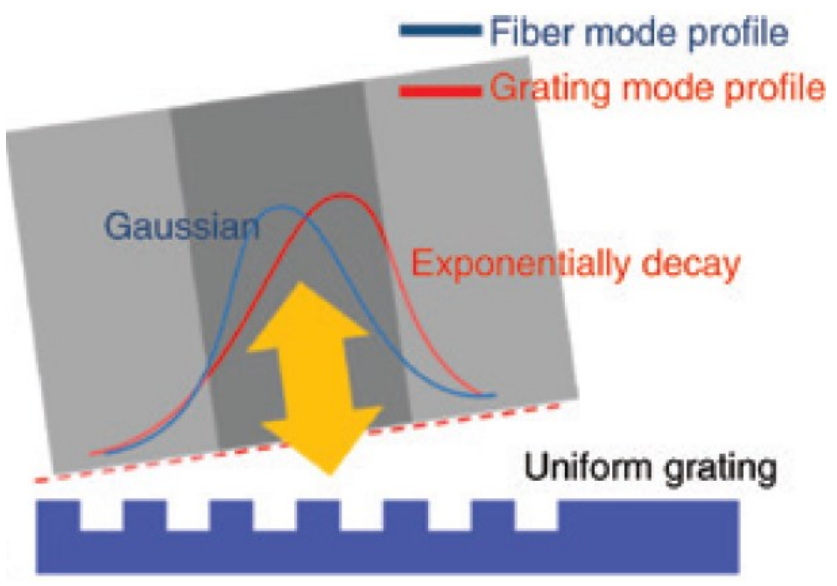

Figure 2.4 Schematic of the field distribution of the light coupled out from the uniform grating coupler [7]

On the other hand, when the light couples from the fiber to the waveguide, the gratings far from the output port will have lower loss than the gratings near the output port.

Because of the significant refractive index difference between silicon and $\mathrm{SiO}_{2}$, the fraction of power diffracted into the substructure cover also needs to be noticed.

\subsubsection{Optimization techniques for grating coupler}

There are three techniques usually used to optimize the performance of the grating coupler: subwavelength gratings (SWGs) structure [42], apodized structure [26], and focusing structure [16].

Subwavelength gratings structure is a structure with a dimension of gratings smaller than the wavelength. Those gratings are small enough to suppress the diffraction effects 
caused by their periodicity. For the subwavelength structure, the dimension has a maximum value of $\frac{\lambda_{0}}{n_{\text {eff }}}$ (which is around $400 \mathrm{~nm}$ for a free space $1550 \mathrm{~nm}$ light in a silicon waveguide) and its grating period usually has an order of $\frac{\lambda_{0}}{10}$ which is around $150 \mathrm{~nm}$ [43].

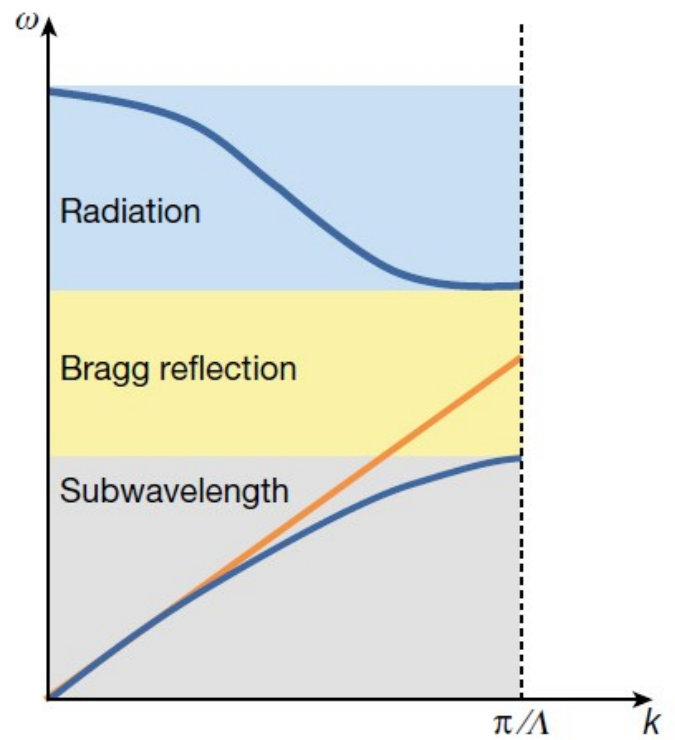

(a)

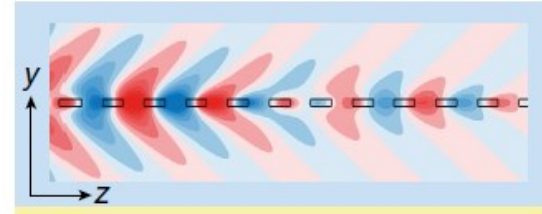

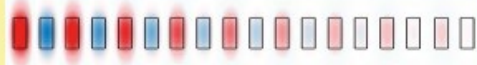

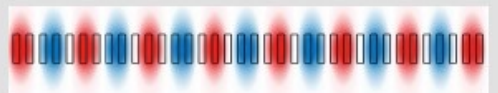

Homogeneous waveguide

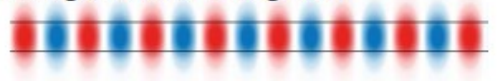

Figure 2.5 Schematic (a) dispersion diagram and (b) corresponding electric field profiles of a periodic slab waveguide for the three regimes of subwavelength-guided wave propagation, Bragg reflection, and radiation. Ref. [44]

Figure 2.5 shows the dispersion relation of the slab waveguide with a periodic grating structure. The orange line is the dispersion of a homogeneous waveguide with an equivalent core refractive index. As the period length decreases, the radiation and diffraction effect will be weaker. In the subwavelength region, the field profile is almost the same as the homogeneous waveguide profile. It shows clearly that the subwavelength structure has a dispersion relation similar to a continuous homogeneous waveguide [44].

By varying the fill-factor, numbers of periods, and the period length of the subwavelength structure, the effective refractive index can be controlled. The SWGs have 
been widely used in grating coupler design with broadband [45], polarization insensitivity [31], and wavelength/polarization splitting property [24, 27].

An apodized structure is widely used to figure out the mode mismatch between the diffracted wave and the fiber mode.

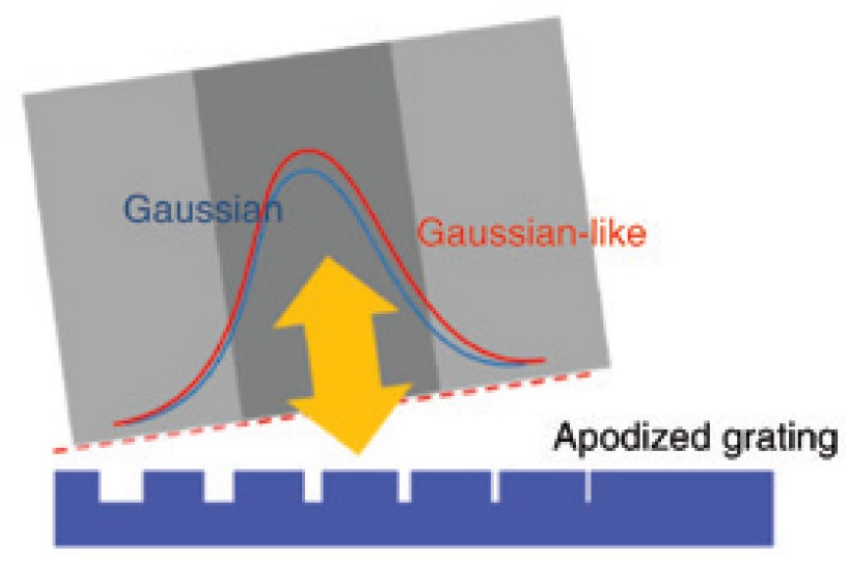

Figure 2.6 schematic diagram of the diffractive field of an apodized grating coupler [7]

As Figure 2.6 shows, the diffractive output field is close to a Gaussian shape. By adjusting the fill-factor, etched depth, or period length for each element of the gratings, the average effective index of the gratings can be varied [7]. From equation 2.8, with a varied average effective index, the center operation wavelength would be changed too. The coupling efficiency will be changed for every single period in the apodized structure, resulting in a Gaussian-like diffracted mode profile. Because of the fabrication difficulty, varying the fill-factor and period length are two ways that are most often used in the grating coupler design. Because of the change of the effective index, part of the back reflection can also be avoided.

Because of the significant dimension difference between the fiber diameter and the silicon waveguide, the straight grating coupler usually has a waveguide width close to the fiber core diameter. At the output port of the grating coupler, a taper will be applied to 
convert the wide waveguide to a silicon wire suited for a single mode. The general straight grating coupler with a taper will have a large footprint and low coupling efficiency. A focusing grating structure can integrate the tapper and grating coupler while decreasing the footprint and increasing the coupling efficiency [46].

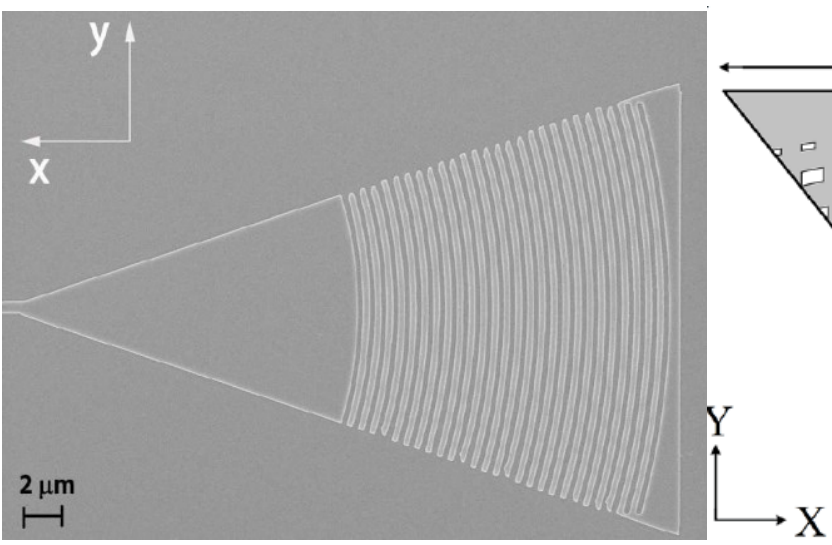

(a)

\section{$\mathrm{Wg}$}

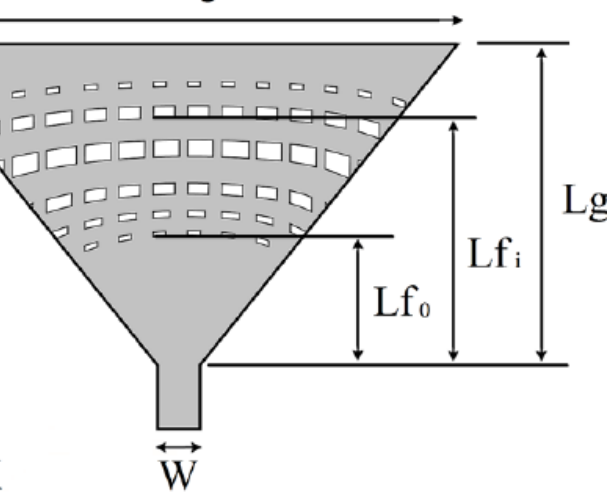

(b)

Figure 2.7 schematic diagram of (a) a classic focusing grating coupler [6] and (b) and apodized focusing grating coupler with a subwavelength structure $[8]$

Figure 2.7 (a) shows a classic structure of the focusing grating coupler [6]. And for the focusing grating structure, the Bragg condition will be [16]

$$
n_{\mathrm{eff}} k r-n_{c} k r \sin \theta \cos \Phi=2 \pi N
$$

where $r$ is the distance of an arbitrary point in the plane of the slab, $\Phi$ represents the angle subtended to the mode propagating direction, and $\mathrm{N}$ is an integer.

In many designs as figure 2.7 (b) [8] shows, subwavelength structure, apodized structure, and focusing structure are used at the same time to achieve a design purpose and get the best result. 


\subsection{Effective Index Approximation}

In the most fundamental equation of the grating coupler (Bragg condition), one crucial parameter is the average effective index of mode propagating in the grating structure.

For an SOI platform, because the thickness of the silicon waveguide is much thinner than the BOX layer (about 10 times thinner), the effect of the silicon substrate can be ignored. Then the $\mathrm{SOI}$ can be thought as a structure with a wave-guiding film layer, a $\mathrm{SiO}_{2}$ substructure layer, and a cladding layer (which may be air or $\mathrm{SiO}_{2}$ ).

The cross-section of a slab is rectangular, so the structure of the waveguide for effective index analysis will be similar to figure 2.8 .

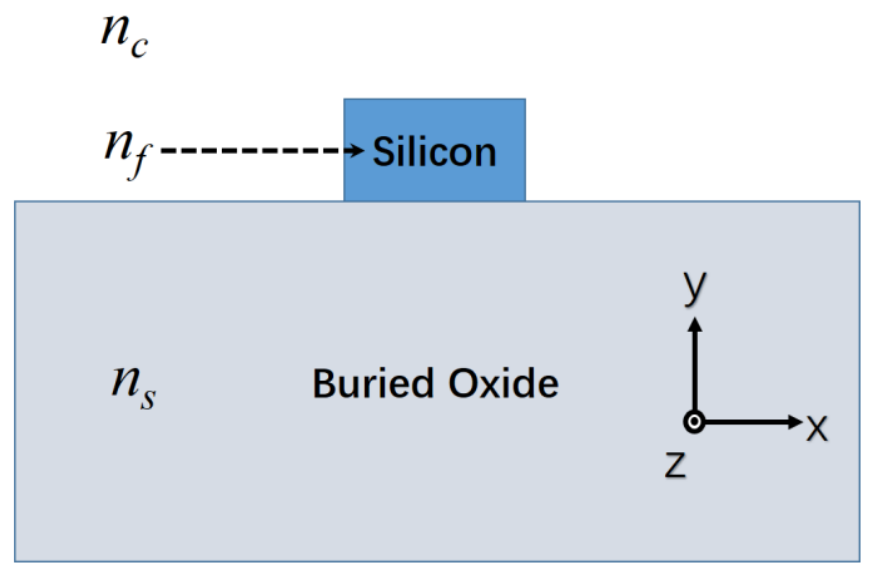

Figure 2.8 schematic diagram of the waveguide structure for effective index analysis

\subsubsection{One-Dimension effective index approach}

Since the cross-section of the grating waveguide has a width of $10 \mu \mathrm{m}$, which is much larger than its thickness, a 1-D effective index can be approached. 


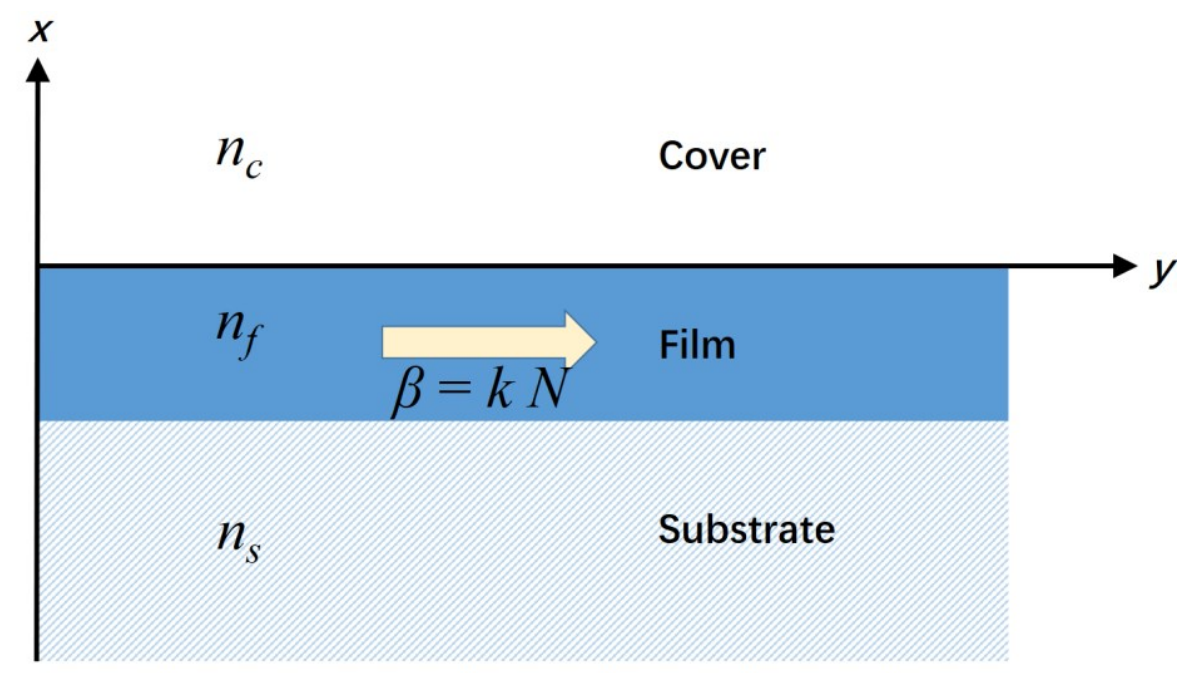

Figure 2.9 schematic diagram of a 1-D planar waveguide

Figure 2.9 shows the structure of the 1-D step-index planar waveguide. By applying Maxwell's equations and Boundary condition, the dispersion relations of the TE and TM modes in this structure can be expressed [55]:

$$
\begin{gathered}
\mathrm{V} \sqrt{1-\mathrm{b}}=\tan ^{-1} \sqrt{\frac{a+b}{1-b}}+\tan ^{-1} \sqrt{\frac{b}{1-b}}+m \pi \\
\mathrm{V} \sqrt{1-\mathrm{b}}=\tan ^{-1} \frac{1}{d} \sqrt{\frac{a+b}{1-b}}+\tan ^{-1} \frac{1}{c} \sqrt{\frac{b}{1-b}}+m^{\prime} \pi
\end{gathered}
$$

Where $\mathrm{V}$ denotes the normalized frequency (known as $\mathrm{V}$ number), $b$ denotes the normalized effective index, $a$ represents the asymmetry parameter, $c$ and $d$ are the index ratios at boundaries only needed for TM, they are defined as [55]:

$$
\begin{array}{r}
\mathrm{V}=k h \sqrt{n_{f}^{2}-n_{s}^{2}} \\
b=\frac{n_{\mathrm{eff}}^{2}-n_{s}^{2}}{n_{f}^{2}-n_{s}^{2}} \\
a=\frac{n_{\mathrm{s}}^{2}-n_{\mathrm{c}}^{2}}{n_{f}^{2}-n_{s}^{2}}
\end{array}
$$




$$
\begin{aligned}
& c=\frac{n_{\mathrm{s}}^{2}}{n_{f}^{2}} \\
& d=\frac{n_{\mathrm{s}}^{2}}{n_{f}^{2}}
\end{aligned}
$$

where $h$ is the thickness of the waveguide, $k$ is the propagating constant, $n_{f}$ is the refractive index of the film layer, $n_{s}$ is the refractive index of the substrate layer, $n_{c}$ is the refractive index of the cover layer, and $n_{\text {eff }}$ is the effective index of the mode propagating in the waveguide. In the above equations, $m$ and $m$ ' are the ordinal number of the TE and TM polarizations. The difference between $m$ and $m$ ' is, for the TM polarization, the fundamental mode $\mathrm{TM}_{0}$ may not exist while $m^{\prime}=0$. For symmetric $\left(n_{\mathrm{c}}=n_{\mathrm{s}}\right)$ waveguides, $a$ is equal to 0 .

For a given waveguide structure with known thickness and material, its V number will only depend on the wavelength of the operating wavelength. In this case, while $a, c$, and $d$ will be constant, $b$ would only depend on the effective index of the mode propagating in the waveguide. The effective index versus the thickness of an SOI platform can be plotted as seen in figure 2.10.

Figure 2.10 is calculated based on the 1-D approximation with an operating wavelength of $1550 \mathrm{~nm}$ and converts $V$ and $b$ into the thickness and effective index. From this plot, we can find that the minimum thickness that can support second TE and TM modes are around $250 \mathrm{~nm}$. Because the 1-D effective index approximation has an infinity width of the waveguide, the $220 \mathrm{~nm}$ thickness of the silicon with limited width is the bestdesigned thickness for an operating wavelength around $1550 \mathrm{~nm}$ in reality. In the meantime, a thicker waveguide can support more modes. 


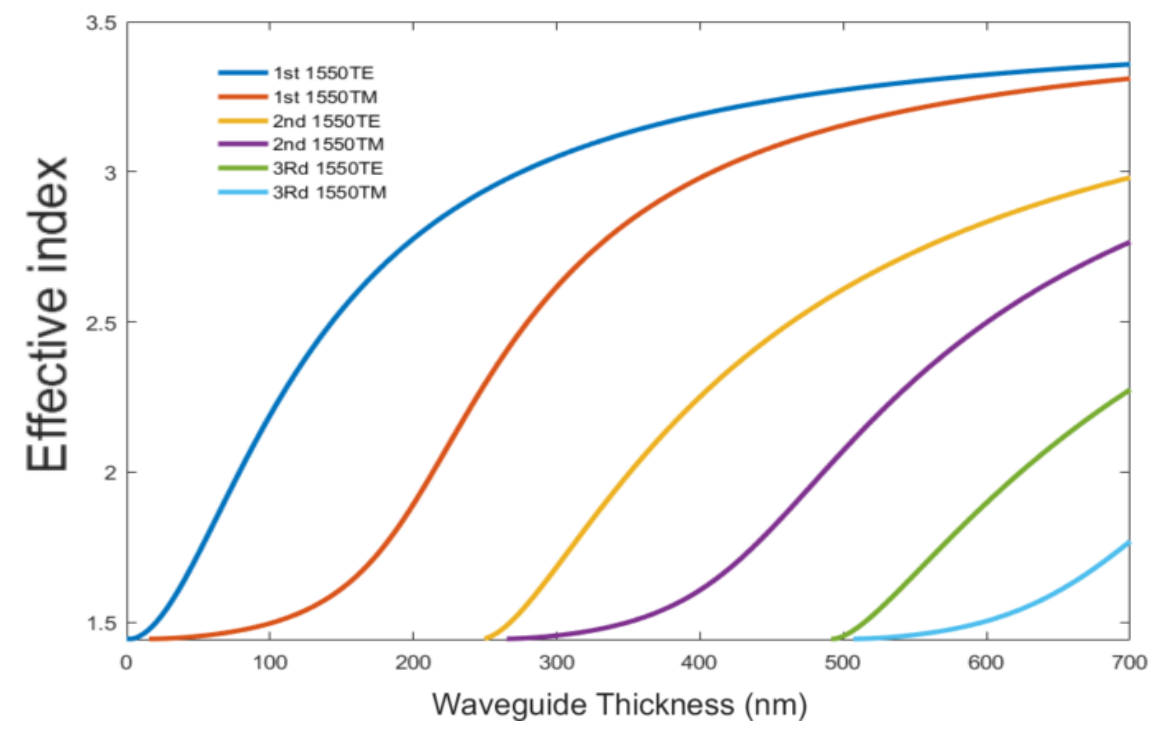

Figure 2.10 The effective index versus thickness of an SOI platform structure

\subsubsection{Effective Index Method}

Although the 1-D effective index approximation has a close result to the real 2-D waveguide with a wide width, the 2-D effective index approximation is still necessary for a more accurate design.

The Effective Index Method (EIM) is an analytical method used for rectangular waveguides such as rib waveguides and strip waveguides. EIM replaces a two-dimensional waveguide with several one-dimensional calculations. In detail, it divides the 2-D structure into a number of one-dimensional regions. Every region will be replaced by its equivalent effective index, and build a new 1-D waveguides approximation and calculate its effective index. 

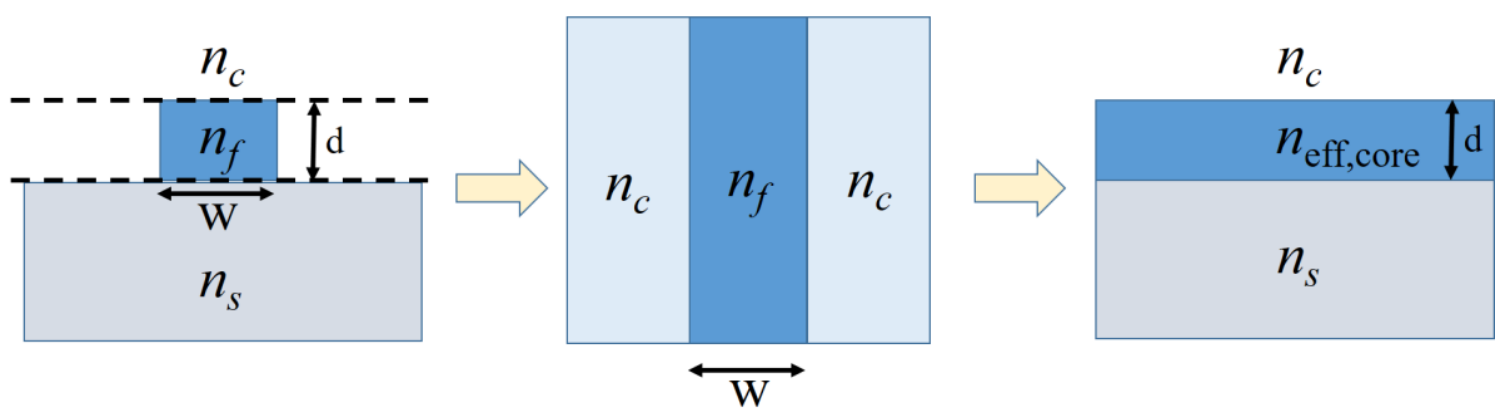

(a)
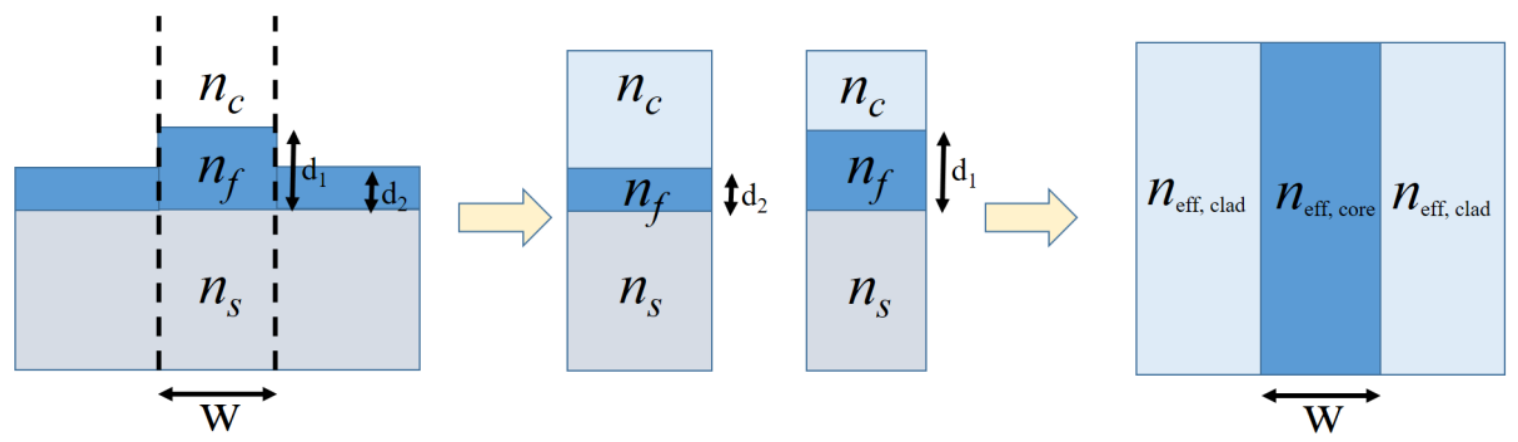

(b)

Figure 2.11 the Effective Index Method analysis steps for (a) a strip waveguide and (b) a rib waveguide.

As figure 2.11 shows, the 2-D waveguide structure has been converted to several 1-D waveguide calculations. For the strip waveguide, as figure 2.10 (a) shows, the 2-D waveguide has been divided into three regions. The upper and bottom regions are kept the same because there is no wave-guiding part in it. In the middle region, the 1-D effective index approximation is applied to get an effective index. This calculated effective index will be used as the core refractive index of the continuous wave-guiding layer that replaces the middle region. Thus, the new 1-D waveguide structure can drive another effective index, which is the approximate effective index of the original 2-D strip waveguide.

EIM can analyze a more complicated structure such as rib waveguide too. The only difference is, for every divided region, the 1-D effective index would be applied. 
Particularly, the effective index calculated for a horizontal structure should apply another polarization equation with the polarization used in a usual vertical structure. For example, the $n_{\text {TEeff }}$ calculated in the second step shown in figure 2.11(a) will use the $n_{\text {TMeff, core }}$ in the third step, while the $n_{\text {TMeff }}$ in the second step will use the $n_{\text {TEeff, core }}$ in the third step.

Because the width of a waveguide is usually longer than its thickness, the effective index of TE polarization is always larger than the effective index of TM polarization. This can be easily proved by applying the boundary condition. A Nanowire structure with a square cross-section will have an almost identical effective index for TE and TM polarizations.

By applying EIM, a three-dimensional structure can also be converted into a twodimensional structure. The Effective Index Method has been widely used, not only in rectangular waveguides, but also in optical fiber [47] and waveguide arrays [48].

\subsubsection{Effective Medium Theory}

In section 2.2.2, the subwavelength structure was introduced. Because this unique structure has a dimension smaller than the operating wavelength and has been shown to be a continuous homogeneous material, the effective index approximation for 1D and EIM cannot be applied to this structure directly.

The Effective Medium Theory was proposed for a finely stratified medium with electromagnetic properties [49]. It can describe the macroscopic properties of composite materials. The effective index theory can only provide an approach-not exact result values - because of the inhomogeneous and complicated properties of different materials. The EMT can be applied to the subwavelength gratings structure. 


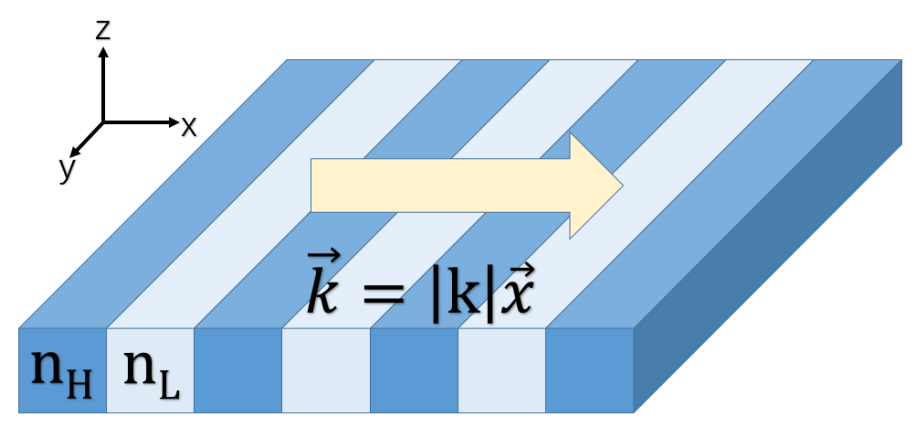

Figure 2.12 Schematic diagram of a wave propagating perpendicular to the alternation media

For conventional gratings with a period length longer than the wavelength, the light will propagate perpendicular to the alternation media as figure 2.12 shows. The $n_{\mathrm{H}}$ and $n_{\mathrm{L}}$ are the reflective index of the alternation media. The alternation media are the gratings. The average effective index of this structure can be calculated by the coupled mode theory and written as [53]:

$$
n_{\text {eff }}=n_{\text {effH }} \cdot \mathrm{ff}+n_{\text {effL }} \cdot(1-\mathrm{ff})
$$

where the $n_{\text {eff }}$ is the average effective index of the gratings, $n_{\text {effH }}$ and $n_{\text {effL }}$ is the effective index of the wave propagating in the media with higher and lower permittivities, ff is the fill-factor.

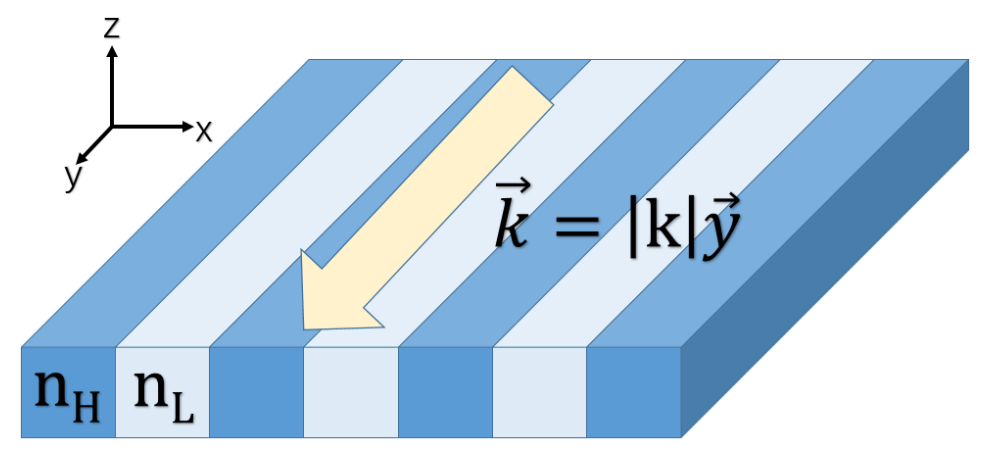

Figure 2.13 Schematic diagram of a wave propagating parallel to the alternation media

On the other hand, if the light propagates parallel to the alternation media (as figure 2.13 shows) the effective index of the wave will follow [53]: 


$$
\frac{1}{n_{\mathrm{eff}}}=\frac{\mathrm{ff}}{n_{\mathrm{effH}}}+\frac{(1-\mathrm{ff})}{n_{\mathrm{effL}}}
$$

However, if the period length is less than the wavelength in the waveguide, the calculation of effective index will be very different from the conventional gratings that have a period length longer than the wavelength.

In the subwavelength region, the permittivity of the media will follow [53]:

$$
\begin{gathered}
\frac{1}{\varepsilon_{\perp}}=\varepsilon_{H} \mathrm{ff}+\varepsilon_{L}(1-\mathrm{ff}) \\
\frac{1}{\varepsilon_{\|}}=\frac{\mathrm{ff}}{\varepsilon_{H}}+\frac{(1-\mathrm{ff})}{\varepsilon_{L}}
\end{gathered}
$$

where $\varepsilon_{\perp}$ and $\varepsilon_{\|}$are the average permittivities for the fields vector parallel and perpendicular to the $\mathrm{xz}$ plane.

For a waveguide that has a period length much smaller than the operation wavelength where $\frac{\lambda_{0}}{n_{\mathrm{effH}}}>>\Lambda$, the zeroth-order approximation can be expressed. If the light propagates parallel to the alternation media, the effective index can be calculated as [31]:

$$
\begin{aligned}
& \frac{1}{n_{\mathrm{TE}}^{(0)}}=\sqrt{\left[\frac{\mathrm{ff}}{n_{\mathrm{L}}^{2}}+\frac{(1-\mathrm{ff})}{n_{\mathrm{H}}^{2}}\right]} \\
& \frac{1}{n_{\mathrm{TM}}^{(0)}}=\sqrt{\left[n_{\mathrm{L}}^{2} \mathrm{ff}+n_{\mathrm{H}}^{2}(1-\mathrm{ff})\right]}
\end{aligned}
$$

where $n_{\mathrm{TE}}^{(0)}$ and $n_{\mathrm{TM}}^{(0)}$ are the zeroth-order refractive indexes of the TE and TM polarizations derived by EMT. Because of the deep-UV fabrication limitation, a 50-nm feature size is hard to realize. If the lateral feature size is of the same order as the operating wavelength in the medium $\left(\Lambda<\frac{\lambda_{0}}{n_{\mathrm{effH}}}\right.$ or $\left.\Lambda \approx \frac{\lambda_{0}}{n_{\mathrm{effH}}}\right)$, the second-order EMT will be employed [31]: 


$$
\begin{aligned}
& \mathrm{n}_{\mathrm{TE}}^{(2)}= \mathrm{n}_{\mathrm{TE}}^{(0)} \sqrt{\left[1+\frac{\pi^{2}}{3}\left(\frac{\lambda_{0}}{n_{\text {effHTE }}}\right)^{2} \mathrm{ff}^{2}(1-\mathrm{ff})^{2}\right]} \\
& \times \sqrt{\left(\mathrm{n}_{\mathrm{L}}^{2}-\mathrm{n}_{\mathrm{H}}^{2}\right)^{2}\left(\frac{\mathrm{n}_{\mathrm{TM}}^{(0)}}{n_{\mathrm{effHTE}}}\right)^{2}\left(\frac{\mathrm{n}_{\mathrm{TE}}^{(0)}}{n_{L} n_{H}}\right)^{4}} \\
& \mathrm{n}_{\mathrm{TM}}^{(2)}=\mathrm{n}_{\mathrm{TM}}^{(0)} \sqrt{\left[1+\frac{\pi^{2}}{3}\left(\frac{\lambda_{0}}{n_{\mathrm{effHTM}}}\right)^{2} \mathrm{ff}^{2}(1-\mathrm{ff})^{2}\left(\frac{\mathrm{n}_{\mathrm{L}}^{2}-\mathrm{n}_{\mathrm{H}}^{2}}{n_{\mathrm{effHTM}} \mathrm{n}_{\mathrm{TM}}^{(0)}}\right)^{2}\right]}
\end{aligned}
$$

where $n_{\mathrm{TE}}^{(2)}$ and $n_{\mathrm{TM}}^{(2)}$ represent the second-order refractive indexes of the TE and TM polarizations derived by EMT.

For the light propagates perpendicular to the alternation media (as figure 2.12 shows), the equations for TE and TM modes switch with each other. Because the effective index strongly depends on the period length of the grating structure, both zeroth-order and second-order EMT are not accurate.

\subsection{Finite Difference Time Domain Method}

Numerical methods are required to analyze and obtain the electric and magnetic solution of the structure with the demand of the complication of the three-dimensional structure.

There are three numerical solution methods commonly used in electromagnetics: the boundary element method (BEM, also known as Method of Moment), the finite element method (FEM), and the finite-difference time-domain method (FDTD). In the optic area, FEM and FDTD are the most used. 
Both of FEM and FDTD can simulate the photonic design and produce an accurate result. In some ways, these two numerical methods are quite similar. However, FEM can handle more complicated geometries but requires much more random-access memory (RAM). FDTD is a time-domain technique where a broadband pulse can be used as the source. It is also easier to implement. FDTD is used for simulating the grating couplers structure in this thesis.

FDTD belongs to the finite difference method (FDM). It uses the time-domain Maxwell's differential equation that can observe the change in the electric field over time and magnetic field in space. This results in the basic FDTD time-stepping relationship where, at any point in space, the updated value of the E-field in time is dependent on the stored value of the E-field and the numerical curl of the local distribution of the $\mathrm{H}$-field in space [50]. The H-field is time-stepped in a similar manner. At any point in space, the updated value of the $\mathrm{H}$-field in time is dependent on the stored value of the $\mathrm{H}$-field and the numerical curl of the local distribution of the E-field in space. Iterating the E-field and $\mathrm{H}$ field updates results in a marching-in-time process wherein sampled-data analogs of the continuous electromagnetic waves under consideration propagate in a numerical grid stored in the computer memory. Kane Yee's seminal 1966 paper proposed spatially staggering the vector components of the E-field and $\mathrm{H}$-field about rectangular unit cells of a Cartesian computational grid so that each E-field vector component is located midway between a pair of $\mathrm{H}$-field vector components, and conversely. 


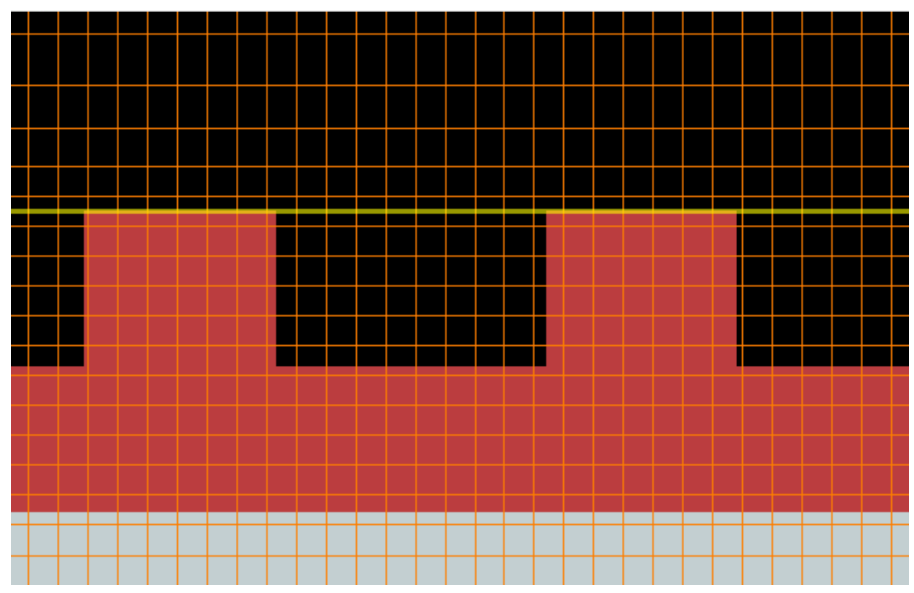

(a)

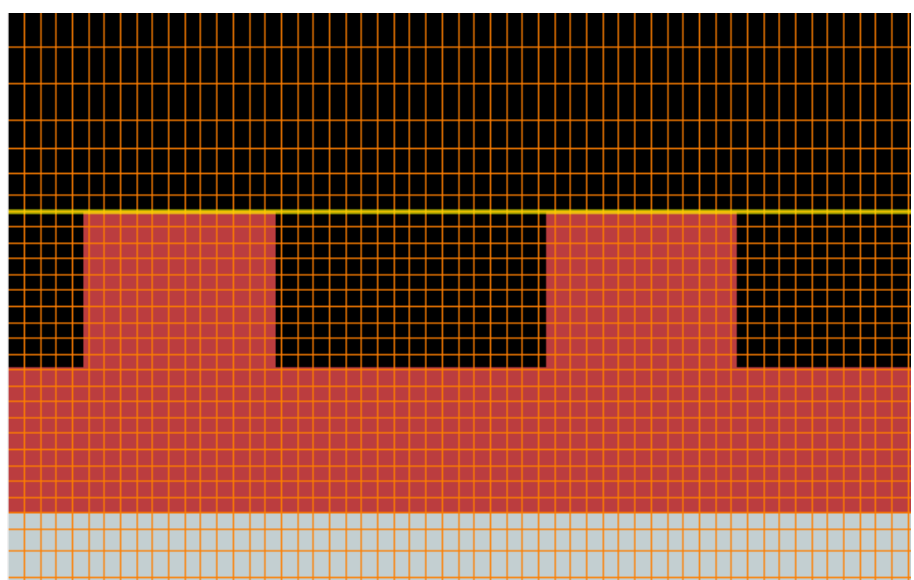

(b)

Figure 2.14 Schematic diagram of a waveguide structure with (a) a poor mesh size and (b) a fine mesh size.

The mesh size (grid size) of the FDTD simulation decides the accuracy and precision of the results. A smaller grid size mesh provides results with higher accuracy. Usually, for a fine simulation result, a mesh size with a maximum one tenth of the operation and the minimum dimension length of the structure should be set. However, a fine mesh size will need a longer calculation time and more computational memory. A poor mesh size might be used for a rough verification of the structure. A result convergent test needs to be done for simulations started from a poor mesh size. 
Figure 2.14 shows examples of a poor and a fine mesh size setting. While figure 2.14 (a) has a minimum mesh size around a quarter of the minimum dimension length, which might not be accurate, (b) shows a fine mesh size setting, and its result should be convergent under this mesh size setting.

Since the 3-D FDTD simulation will take a long time (in the order of hours) compared with the 2-D FDTD simulation (in the order of seconds), a 2.5-D FDTD might be used for the 3-D structure simulation. It can convert a 3-D structure into a 2-D structure and replace the materials in 3-D with equivalent refractive index materials in 2-D. This method can be applied in structures such as direct couplers, ring resonators, MMIs, etc. However, since the equivalent refractive index is not accurate for the TM polarization, and because 2.5-D FDTD cannot apply to a complicated structure, 3-D FDTD simulation is still necessary.

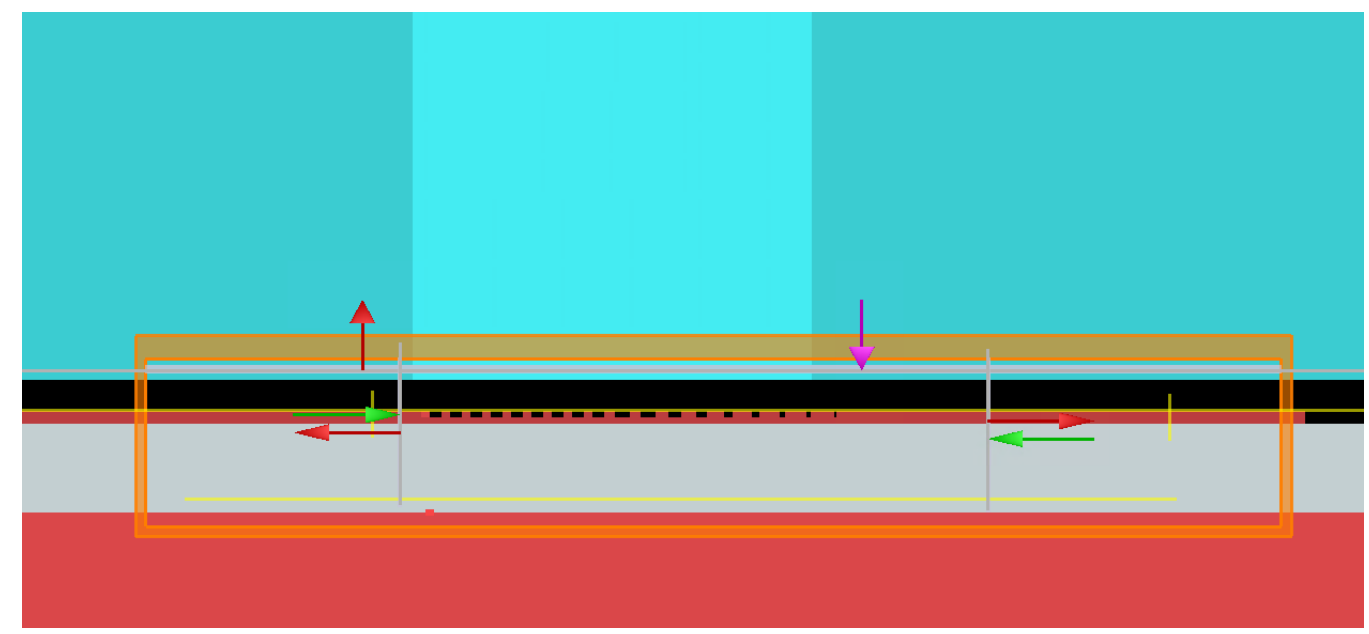

Figure 2.15 Schematic diagram of a vertical incident grating coupler

Lumerical Mode and FDTD Solution are used in this thesis. Lumerical Mode is used to calculate the effective index of a waveguide by the FDM. Lumerical FDTD Solution is the tool used to simulate the input and output performances of grating couplers. 
Figure 2.15 shows a schematic diagram of a vertical incident grating coupler. In this example diagram, the red and gray blocks are the waveguide structure of the grating coupler. Blue blocks represent the cross-section of a single-mode fiber. Yellow lines are the power monitors that can calculate the electric and magnetic fields at particular positions. Green and red arrows are the input or output ports that can work as a light source. The orange frame is the boundary of the simulation region.

Most of the time, Perfectly Matched Layer (PML) boundary conditions were used for all the axes in the FDTD simulations. PML boundaries are implemented as an absorbing material that is also impedance matched to the surrounding materials to minimize reflections. In the boundary condition, the symmetric condition may also be chosen to shorten the simulation time. 


\section{Chapter 3:}

\section{Vertical Incident Dual-wavelength}

\section{Grating Coupler}

Low-cost dual-band (O-band and C-band) chip transceivers are required because of an increasing demand for wavelength-division-multiplexing (WDM) systems in the fiberto-the-home network (FTTH) services. In the work people have already done, dual-band wavelength splitting grating couplers with a small incident angle need more postprocessing and packaging than the vertical indent grating couplers. However, in all the vertical incident dual-wavelength grating couplers that have already been demonstrated, nonuniform gratings processed by the inverse design or a gradient-based algorithm either have a low transmission or a small feature size of $50 \mathrm{~nm}$. In this chapter, the design flow for a novel dual-band grating coupler with a vertical incident will be presented. The chapter begins with the design for a general grating coupler. Following that, the vertical incident grating coupler and its special performances are discussed. At the end of this chapter, the vertical incident dual-wavelength grating coupler are shown and optimized.

\subsection{Shallow Etched Grating Coupler Designed}

Due to the inherent difference between the TE and TM polarizations, the coupling efficiency of TM polarization is lower than TE polarization in most cases. Also, shallow etched grating couplers are used because they usually have a lower coupling loss and back 
reflection. In this chapter, the grating coupler is shallow-etched and designed for the fundamental TE mode $\left(\mathrm{TE}_{0}\right)$.

In the 2-D simulation design, an infinite width (z-axis) is assumed. The effective indexes in the structure will follow from the result of a 1-D effective index approximation. In any grating coupler design, the Bragg condition must be satisfied.

Since the grating coupler design is based on the SOI platform, the Bragg condition (equation 2.8) can be rewritten as:

$$
n_{\text {aveeff }}-n_{\mathrm{SiO}_{2}} \sin \theta=\frac{\lambda_{0}}{\Lambda}
$$

where $n_{\text {aveeff }}$ is the average effective index of the gratings, $n_{C}$ is replaced by $n_{S i O_{2}}$ since a $\mathrm{SiO}_{2}$ cover is assumed. By applying the coupled mode theory with equation $2.17, n_{\text {aveeff }}$ would be expressed as :

$$
n_{\text {aveeff }}=n_{\text {effH }} \cdot \mathrm{ff}+n_{\text {effL }} \cdot(1-\mathrm{ff})
$$

where $n_{\text {effH }}$ and $n_{\text {effL }}$ are the effective indexes of the filled part and etched part of the gratings. Because the effective index is dependent on the thickness of the waveguide, and most parameters are unknown, an initial condition would be set as shown in Table 3.1.

Table 3.1 Initial values set for the grating coupler

\begin{tabular}{|c|c|c|c|c|}
\hline Thickness of Si & Thickness of SiO2 & ff & Etch Depth & $\boldsymbol{\lambda}_{\mathbf{0}}$ \\
\hline $220 \mathrm{~nm}$ & $2 \mu \mathrm{m}$ & 0.5 & $100 \mathrm{~nm}$ & $1550 \mathrm{~nm} / 1310 \mathrm{~nm}$ \\
\hline
\end{tabular}

The refractive index of silicon for $1550 \mathrm{~nm}$ and $1310 \mathrm{~nm}$ are 3.4776 and 3.50685, respectively. At the same time, the refractive index of $\mathrm{SiO}_{2}$ is set as 1.444 and 1.44672 to $1550 \mathrm{~nm}$ and $1310 \mathrm{~nm}$, respectively. With those values shown above, the average effective 
index of the grating can be calculated by the 1-D effective index approximation. Table 3.2 shows the effective index of both $1310 \mathrm{~nm}$ and $1550 \mathrm{~nm}$.

Table 3.2 Calculated effective index

\begin{tabular}{|c|c|c|c|}
\hline Wavelength & $\boldsymbol{n}_{\text {effH }}$ & $\boldsymbol{n}_{\text {effL }}$ & $\boldsymbol{n}_{\text {aveeff }}$ \\
\hline $1550 \mathrm{~nm}$ & 2.849052 & 2.345348 & 2.597200 \\
\hline $1310 \mathrm{~nm}$ & 2.994441 & 2.518829 & 2.756635 \\
\hline
\end{tabular}

To ensure the accuracy of the calculated effective, Lumerical Mode is used to simulate the effective index.

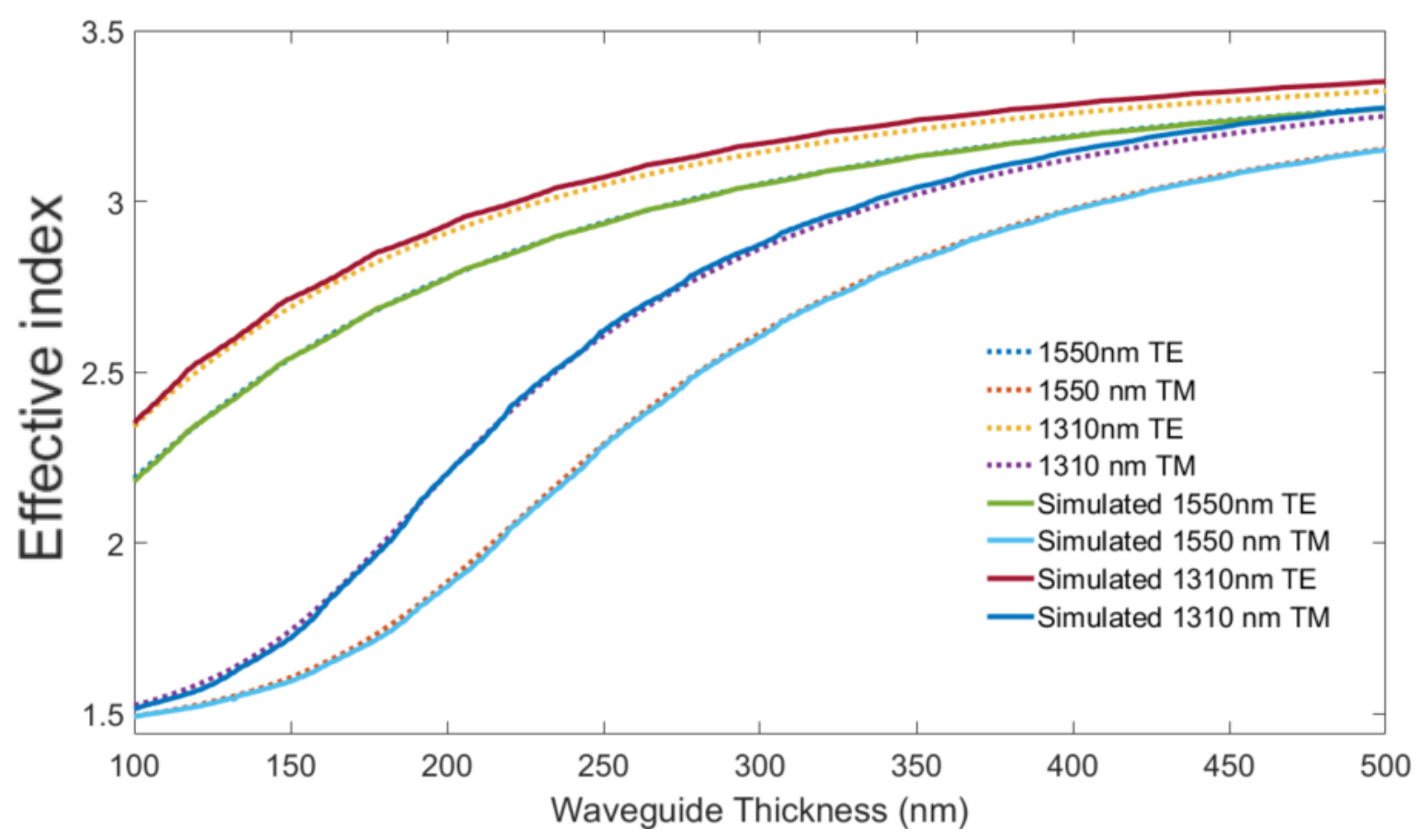

Figure 3.1 The calculated and simulated effective index versus waveguide height

From figure 3.1, the simulated effective indexes and calculated effective indexes are identical with each other. These approximations have a maximum error of $\sim 1 \%$. Since the Lumerical Mode simulation is a 2-D effective approximation calculated by a 2D FiniteDifference Eigenmode Solver with a finite width of the waveguide (which is $10 \mu \mathrm{m}$ in 
simulation), the error is acceptable. For a 2D grating structure with a known thickness and width of $\sim 10 \mu \mathrm{m}$, the 1-D effective approximate result can be used directly in the 2-D grating coupler design.

Since the fill-factor, operation wavelength, average effective index, waveguide thickness, and etch depth are known, the incident angle and period length only depend on each other. Thus, an incident angle versus period length plot can be shown by applying equation 3.1:

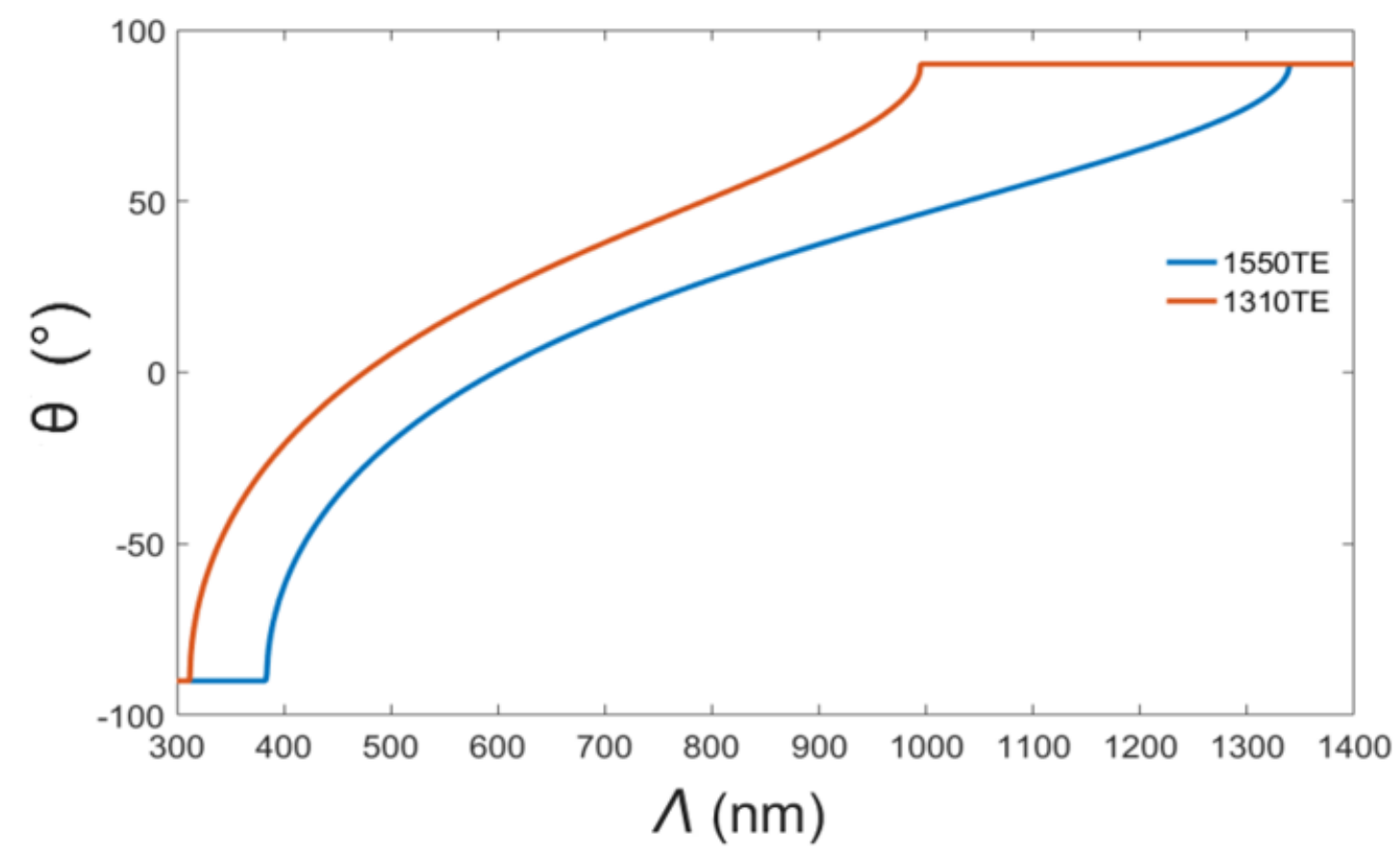

Figure 3.2 The incident angle versus period length for $1550 \mathrm{~nm}$ and $1310 \mathrm{~nm} \mathrm{TE}_{0}$ mode

Afterward, the incident angle and period length can be decided within the plot directly. For example, a 25-degree incident angle and $779.4 \mathrm{~nm}$ pitch length for operation wavelength $1550 \mathrm{~nm}$ can be chosen. From figure 3.2, all the parameters for a 2-D grating coupler can be confirmed. Figure 3.3 shows the grating coupler model in Lumerical FDTD Solutions. 


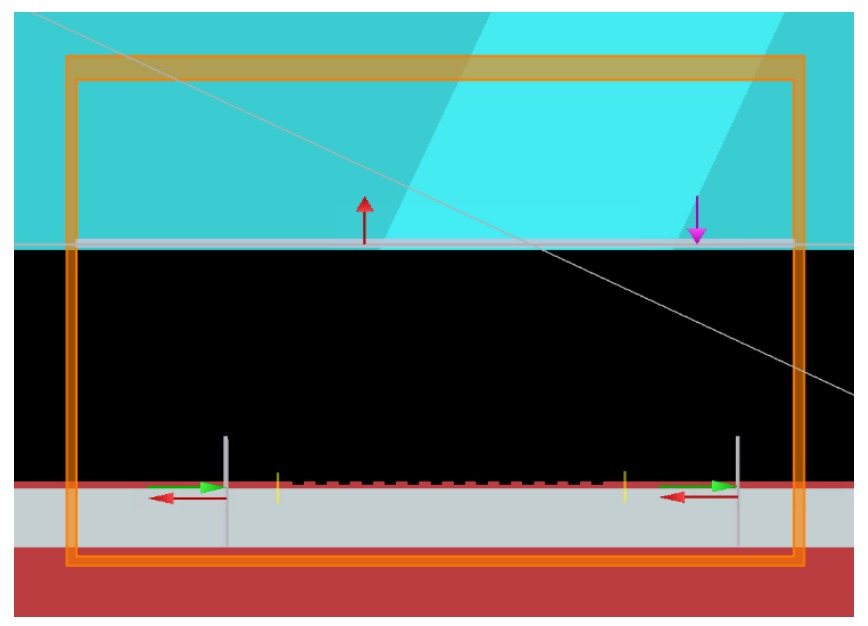

Figure 3.3 The example grating coupler in Lumerical FDTD Solutions

In figure 3.3, the simulation region (orange square), there is a large gap between the fiber (blue blocks) and the waveguide (thin red layer) because the FDTD needs a space at least longer than one wavelength to simulate the propagating mode. Also, the whole BOX layer (gray block) is included in the simulation region to achieve the most accurate result.

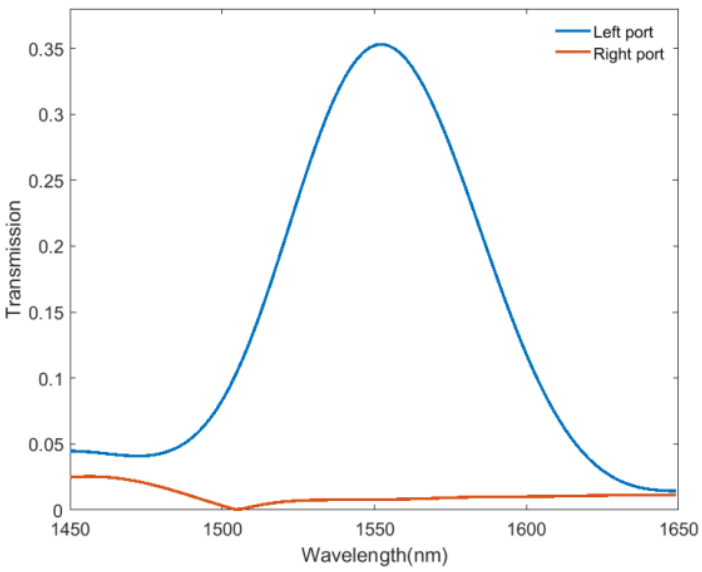

(a)

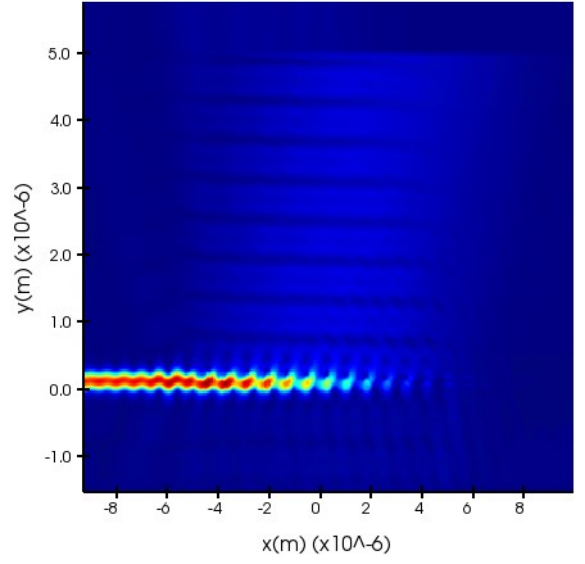

(b)

Figure 3.4 (a)The transmission spectrum and (b) power profile of the example grating coupler

Figure 3.4 shows the transmission spectrum and power profile of this designed grating coupler. The transmission is defined as the ratio between the power coupled into the waveguide and the total power from the fiber. Similarly, coupling efficiency is defined 
as the ration between the power of the coupled mode in the waveguide and he total power from the fiber. When the second or higher order diffractions are weak, transmission would be very close to the coupling efficiency. Lumerical uses an overlap analysis to calculate the coupling efficiency [54].

In the example shown, the transmission It has a center wavelength at $1550 \mathrm{~nm}$ exactly as the expectation. This grating coupler has a coupling efficiency of around $35 \%$, a $120 \mathrm{~nm} 3-\mathrm{dB}$ bandwidth, and a back reflection of less than $-15 \mathrm{~dB}$. Although the transmission is not ideal, it can be increased through optimization. The power profile shows most power has coupled into the waveguide at $1550 \mathrm{~nm}$.

In the next sections, the vertical incident grating coupler and its optimization will be shown.

\subsection{Vertical Incident Grating Coupler Designed}

A vertical incident (where incident angle $\theta$ would equal to 0 degrees) grating coupler design can directly get all the design parameters from figure 3.2. However, a small angle of the grating coupler design is always used to avoid the strong back reflection. Second, a vertical incident would have two identical output ports with the same performance. Because of this, a vertical incident grating coupler is also a 3-dB power splitting grating coupler. Figure 3.5 shows a general scheme of a 2-D vertical incident grating coupler [51]. 


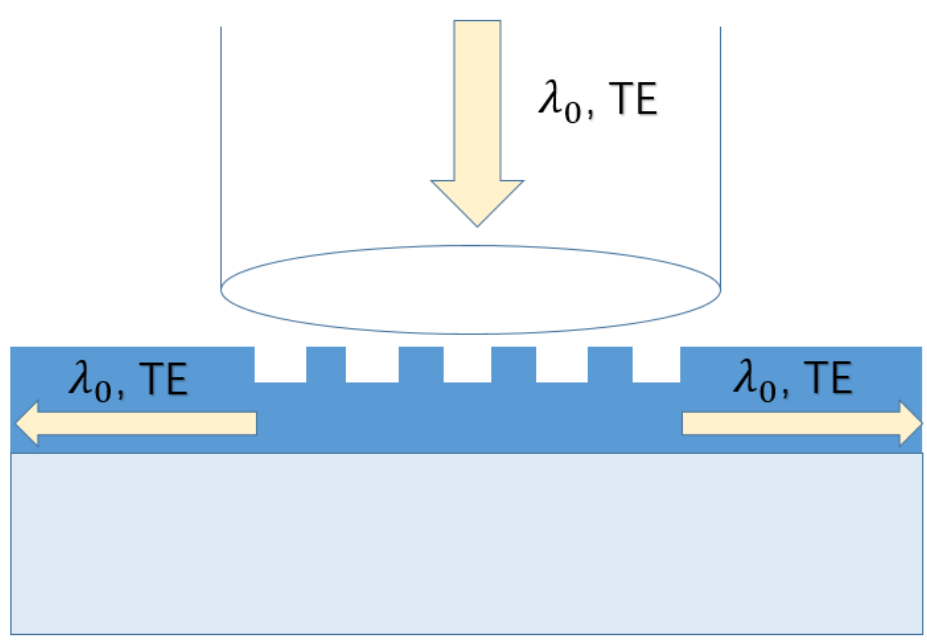

Figure 3.5 Schematic diagram of a vertical incident grating coupler

\subsubsection{Design theory of the vertical incident grating coupler}

The phase match condition (equation 2.3) of a vertical incident grating coupler with $\theta=0^{\circ}$ will be expressed as:

$$
\beta=m K
$$

A fundamental TE mode would follow $\beta=K=\frac{2 \pi}{\Lambda}$ and $n_{\text {aveeff }}=\frac{\lambda_{0}}{\Lambda}$ in this structure. The period length can be written as $\Lambda=\frac{\lambda_{0}}{n_{\text {aveeff }}}=\frac{2 \pi}{\beta}$. Figure 3.6 shows the relationship schematically.

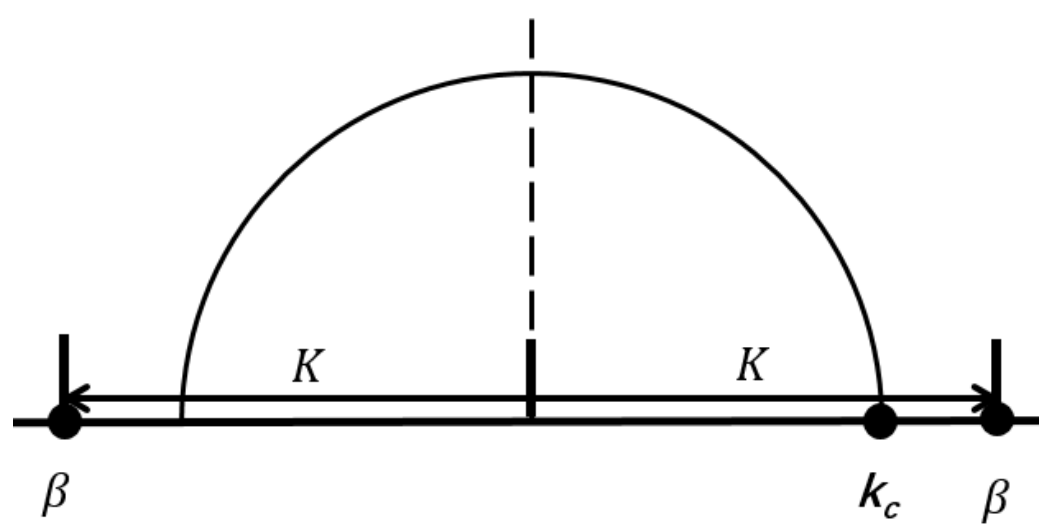

Figure 3.6 Schematic Bragg condition of a vertical incident grating coupler 
However, under this structure condition $\left(n_{\text {aveeff }}=\frac{\lambda_{0}}{\Lambda}\right)$, the grating structure has a strong second-order coupling efficiency inside the waveguide as well. Equation 2.3 can be written as:

$$
\beta-\sin \left(-90^{\circ}\right) \beta=2 \beta=2 K
$$

where both equation 3.3 and 3.4 have a result of $\beta=K$. The diffraction order $m$ in equation 2.3 is equal to 2 and $k_{c}$ is equal to $\beta$ because the diffraction is inside the waveguide. Since $\theta$ is equal to -90 degrees, the coupling inside the waveguide is the backward reflection. For this equation, the Schematic Bragg condition can also be plotted:

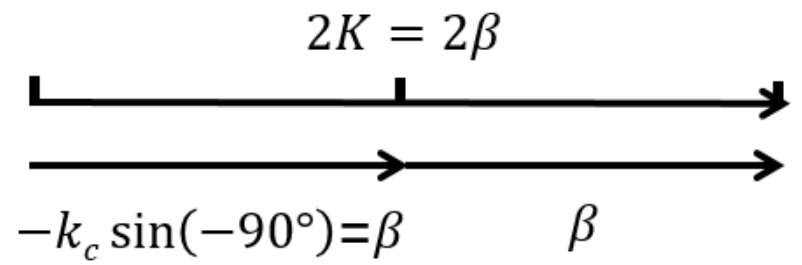

Figure 3.7 Schematic Bragg condition within the waveguide of the vertical incident grating coupler

Because the vertical incident grating coupler has two identical output ports, the back reflection will occur in both directions of the grating waveguide and cancel each other. However, if the fiber position is not at the above of the exact center of the grating coupler, an oscillation transmission spectrum would be observed.

By using a $220 \mathrm{~nm}$ SOI platform with a $100 \mathrm{~nm}$ etch depth and fill-factor of 0.5 , the vertical incident design can be found for both $1310 \mathrm{~nm}$ and $1550 \mathrm{~nm}$. For $1550 \mathrm{~nm}$, the period length would be $596.797 \mathrm{~nm}$, while $475.22 \mathrm{~nm}$ is the period length for $1310 \mathrm{~nm}$. Both wavelengths have a total grating length of around $9 \mu \mathrm{m}$ (the dimension close to the diameter of the fiber core) to increase the coupling efficiency. Afterward, the parameters of this design can be entered into Lumerical FDTD to simulate the performance of the grating coupler. 


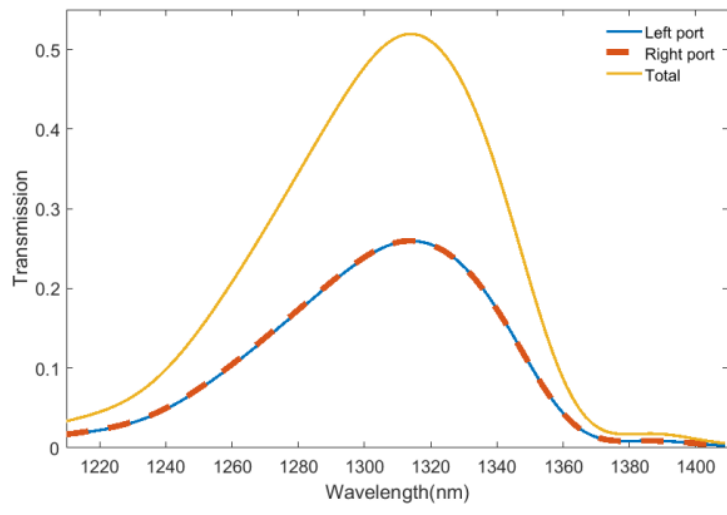

(a)

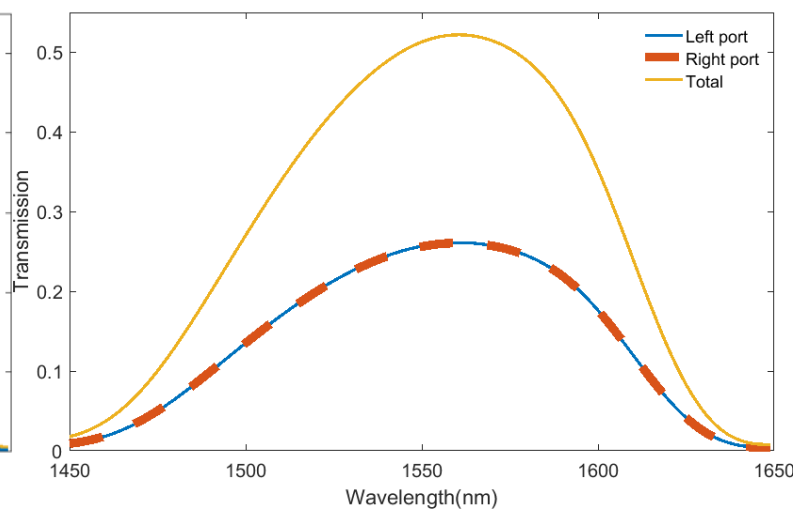

(b)

Figure 3.8 Simulation transmission spectrum for a vertical incident grating coupler at operating wavelengths (a) $1310 \mathrm{~nm}$ and (b) $1550 \mathrm{~nm}$

A transmission spectrum for a grating coupler or direct coupler should have a Gaussian or sinc-functions shape. As figure 3.4 shows, the transmission spectrum is almost a perfect Gaussian-shape. However, the spectrums shown in figure 3.8 for the O-band and C-band are slightly inaccurate. Because the back reflection at the operating wavelength and shorter wavelength is more sensitive, the center wavelength is shifted marginally to the right.

Although there is a back reflection in the gratings, the total transmission is still greater than the design with a $25^{\circ}$ incident angle.

\subsubsection{Parametric Sweeps and Analysis of the Vertical Incident Design}

In 3.2.1, the designs were based on the initial condition without any optimization.

Parametric sweeps and analysis will be applied to achieve a better coupling efficiency.

Since the transmission spectrums for $1310 \mathrm{~nm}$ and $1550 \mathrm{~nm}$ shown in figure 3.8 have similar performances, the parametric sweeps and analysis will only be done with the $1550 \mathrm{~nm}$ design. 


\section{A. Fiber position}

For a grating coupler with a period length that satisfies $\Lambda=\frac{\lambda_{0}}{n_{\text {aveeff }}}$, a second-order coupling inside the waveguide (which is the back reflection) would occur inherently. However, for the vertical incident grating design, the back reflection loss would only be unaffected when the light source is at the exact center of a grating structure. The back reflections in two directions would cancel each other under the condition of the fiber being at the center. Otherwise, if the fiber is not at the center of the grating structure, the back reflection would be powerful and show up immediately after the position shift.

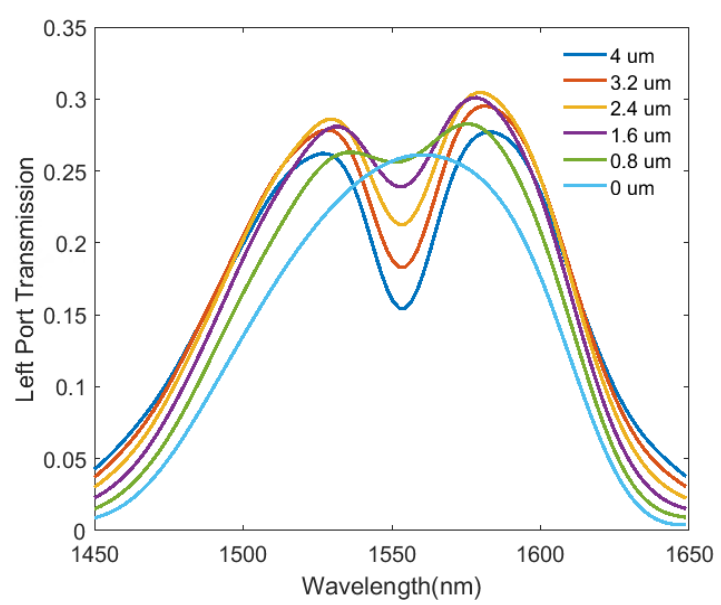

(a)

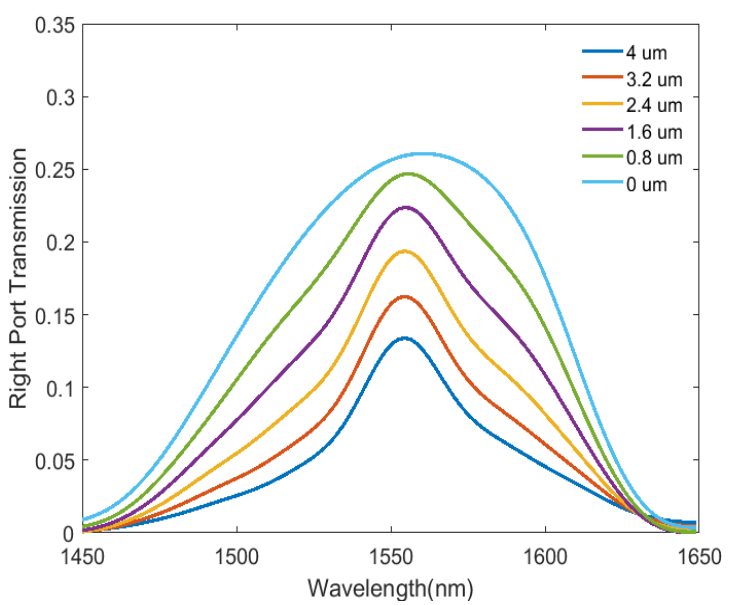

(b)

Figure 3.9 Transmission spectrum for (a) left port and (b) right port with a fiber position shift

From figure 3.9 (a), a prominent curve can be observed at the $1550 \mathrm{~nm}$. This is due to the back reflection in the waveguide. While the left port has a stronger vertical coupling and a strong back reflection loss with the fiber position shifting, the right port has a weaker vertical coupling and a strong back reflection from the left port. The superposition of the coupling and back reflection forms the m-shape and $\wedge$-shape spectrums of the output ports. In the meantime, the total transmission decreases with the fiber position shift. Obviously, the best fiber position is at the center of a grating structure. 


\section{B. Incident angle}

From equation 2.3 and 3.3, since all other parameters are constant, an incident angle change would only affect the center wavelength, and an equation for the incident angle can be written: from the Bragg condition

$$
\theta=\operatorname{asin}\left(-\frac{K}{\beta k_{c}}\right)
$$

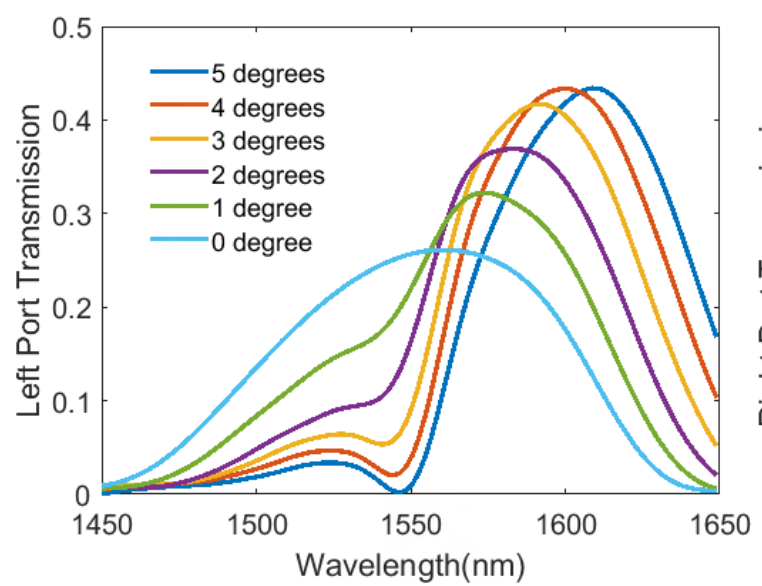

(a)

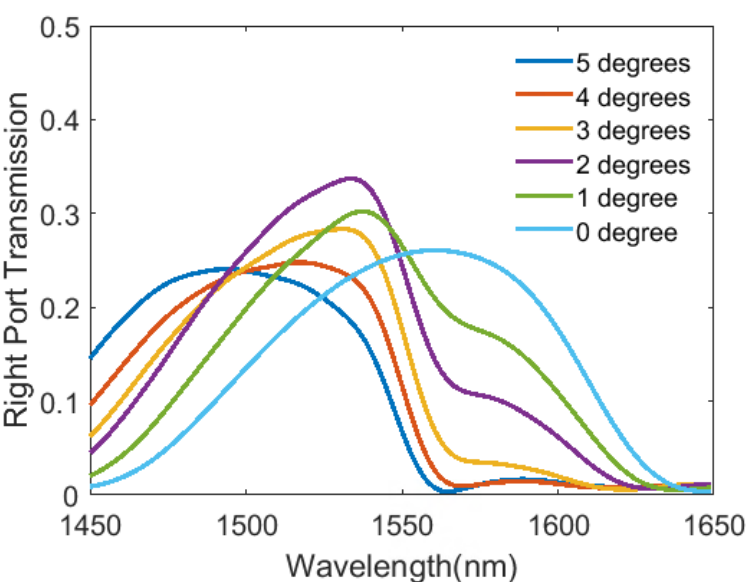

(b)

Figure 3.10 Transmission spectrum for (a) left port and (b) right port with an incident angle sweep

For the coupling with a small incident angle $\theta$, the angle is positive for the forward direction and negative for the backward direction. By applying the Bragg condition for the vertical incident grating coupler structure, while a positive incident angle would have a shorter center wavelength, a negative angle would have a longer wavelength. Figure 3.10 shows the transmission spectrum of the vertical incident grating coupler design with an incident angle sweep of $1550 \mathrm{~nm}$. This grating coupler behaves as a wavelength splitting grating coupler with a small angle incident. A hollow is occurred because of the back reflection around $1550 \mathrm{~nm}$. At the same time, the left part has a lower transmission 
compared with the right port because the shorter wavelength has a stronger second-order diffraction.

\section{Fill-factor and period length}

The fill-factor (also known as duty cycle) is the ratio between the filling width and the period length of the grating structure. It would mainly affect the $n_{\text {aveeff }}$ of the waveguide. For a vertical incident grating coupler with incident angle $\theta=0^{\circ}$, it would have:

$$
\Lambda=\frac{\lambda_{0}}{n_{\text {aveeff }}}
$$

Clearly, for a given grating coupler, the center wavelength would also shift if the fill-factor changes.

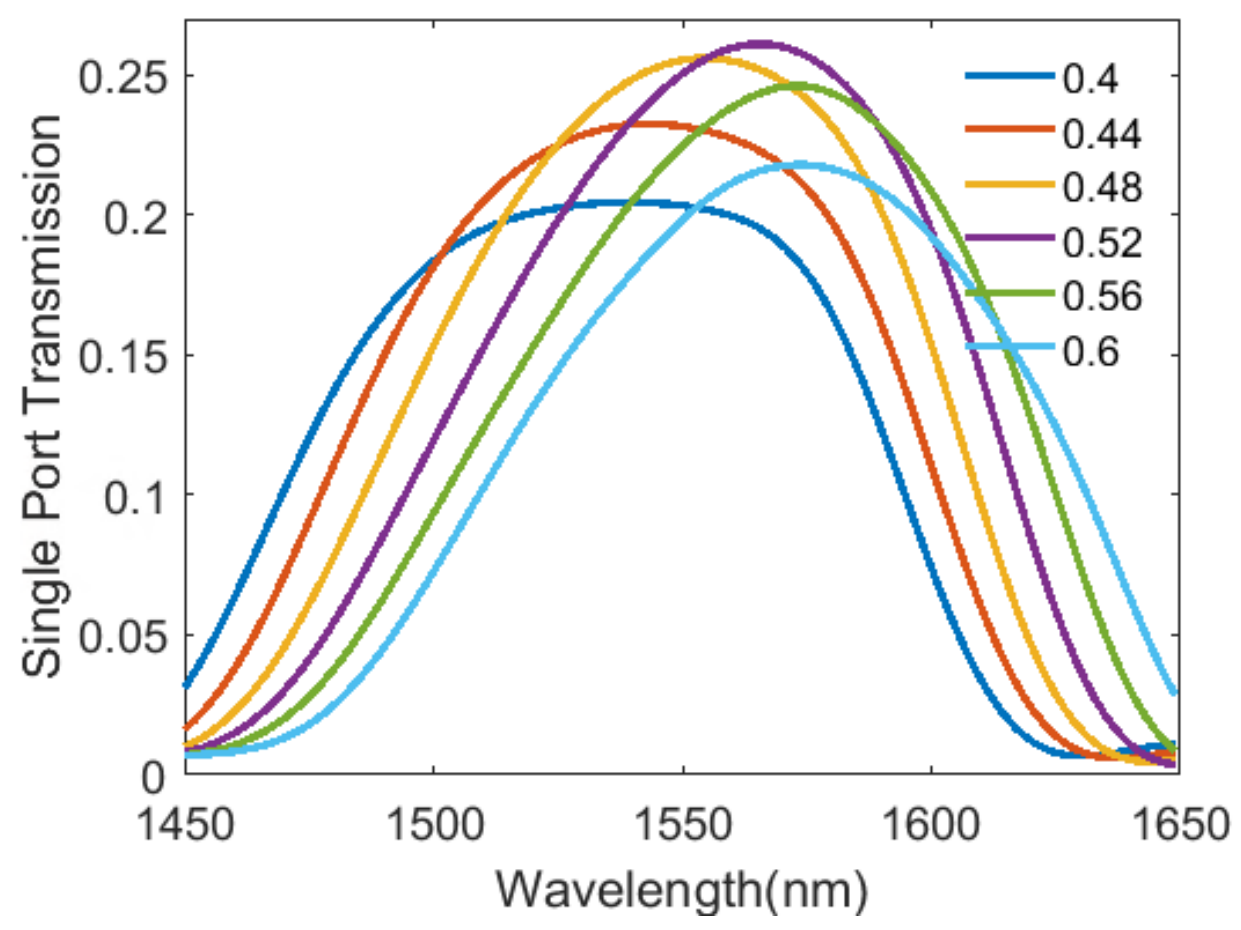

Figure 3.11 Transmission spectrum of the vertical incident grating coupler with a duty cycle sweep 
Figure 3.11 only shows the transmission of a single port because the vertical incident grating coupler has two identical output ports. With a $10 \%$ fill-factor change (about $60 \mathrm{~nm}$ fill-width different), the center wavelength has an acceptable shift of $30 \mathrm{~nm}$. This means a $10 \mathrm{~nm}$ filling width fabrication error would only shift the center wavelength about $5 \mathrm{~nm}$, while the transmission drops $1 \mathrm{~dB}$ (about $20 \%$ ) for a $60 \mathrm{~nm}$ filled width fabrication error. This tolerance is acceptable.

Changing the period length is a more direct way to alter the center wavelength of a vertical incident grating coupler. From equation 3.6, we know that the period length has an inverse relationship with the center wavelength. Unlike changing other parameters that would affect the average effective index, adjusting the period length is directly changing the Bragg space of the structure.

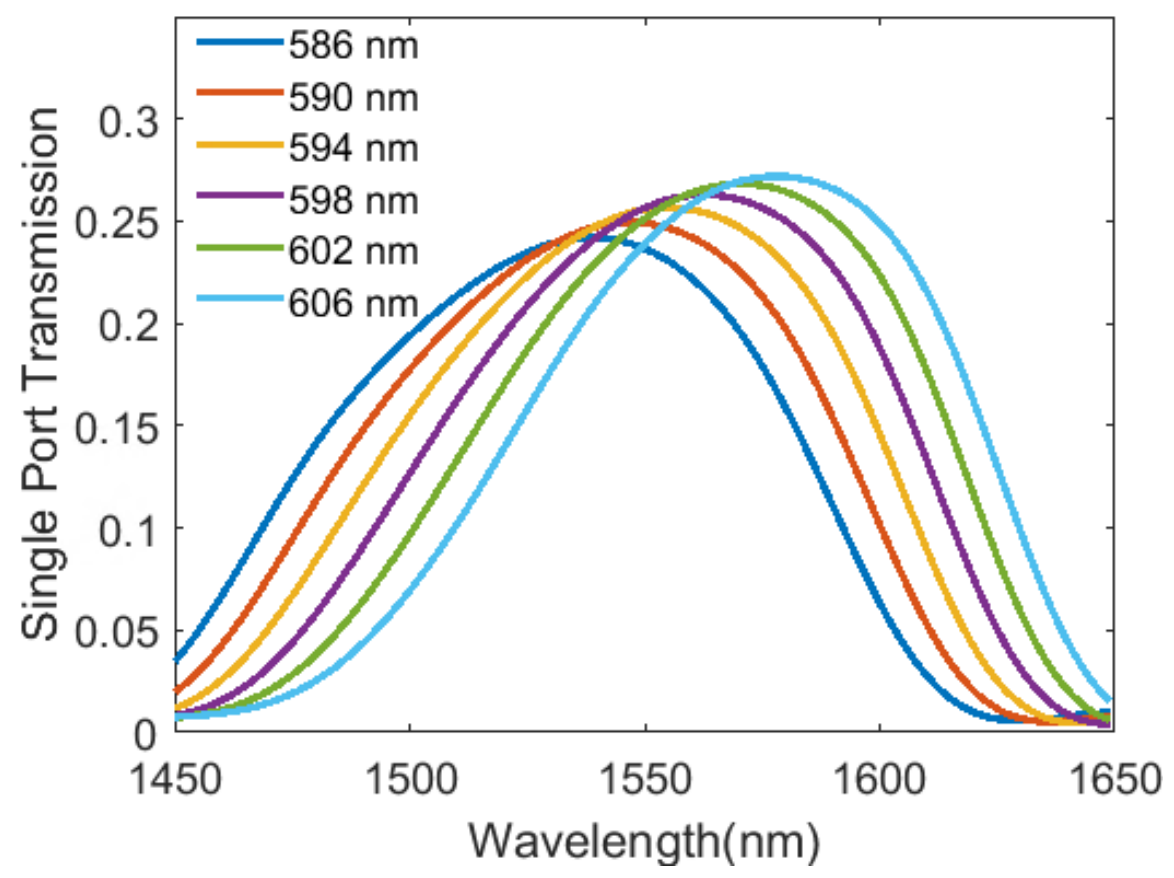

Figure 3.12 Transmission spectrum of the vertical incident grating coupler with a period length sweep 
Figure 3.12 shows the effect of sweeping the period length. For every $10 \mathrm{~nm}$ period length change, the center wavelength would shift about $35 \mathrm{~nm}$ and the transmission would change about $2 \%$.

Basically, sweeping the fill-factor and period length would have a similar effect on the center wavelength because the fill-factor has a linear-like relationship with the average effective index. Both the average effective index and period length have an inverse relationship with the center wavelength.

\section{Etch depth and Waveguide thickness}

Both etch depth and waveguide thickness are related to the average effective index of gratings. The etch depth is related to the $n_{\mathrm{effL}}$ and waveguide thickness is relevant to the $n_{\mathrm{effH}} \cdot$

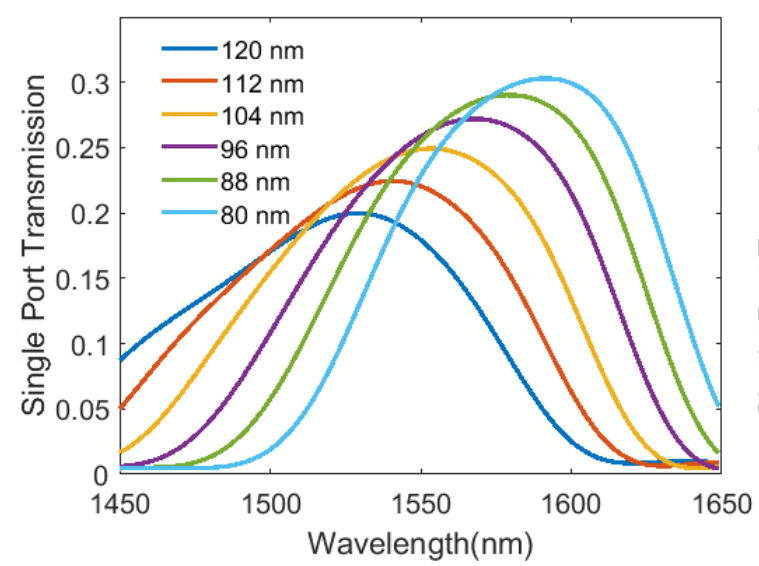

(a)

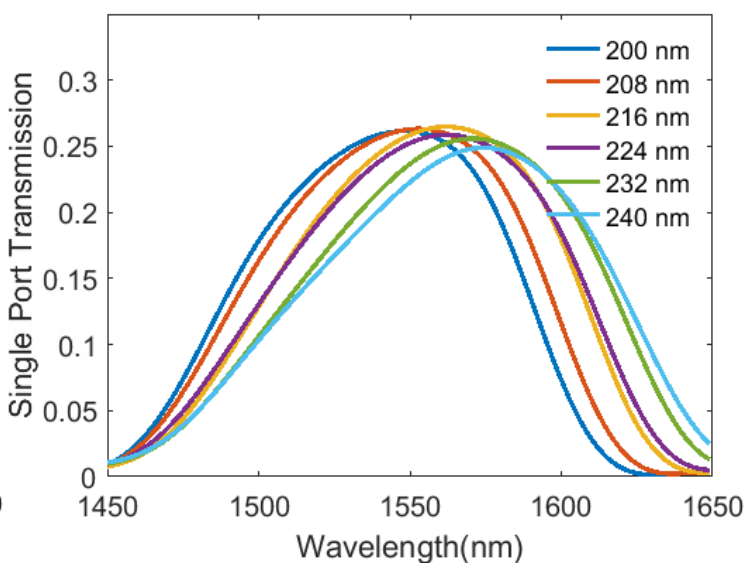

(b)

Figure 3.13 Transmission spectrums of the vertical incident grating coupler with (a) an etch-depth sweep or (b) a waveguide thickness sweep

From figure 3.13 , we find that the waveguide has a larger effective index changing rate at a thinner thickness. This result can be observed in figure 3.1 directly. With the same 8-nm-per-step parameter sweep, sweeping the etch-depth results in a larger center 
wavelength and transmission change than sweeping the wavelength thickness. Also, by decreasing the etch depth, the transmission increased substantially because a lower etch depth increases the average effective so that the waveguide can be coupled with more light inside the waveguide. A smaller effective index between the etch and fill part of a grating would decrease the back reflection as well.

\section{E. Number of periods (N)}

Usually, a fiber-to-chip grating coupler should have a long enough length (close to the fiber core diameter) to maximize the coupling efficiency. However, a more extended grating of the vertical incident grating coupler would not have a maximum coupling efficiency because of the back reflection.

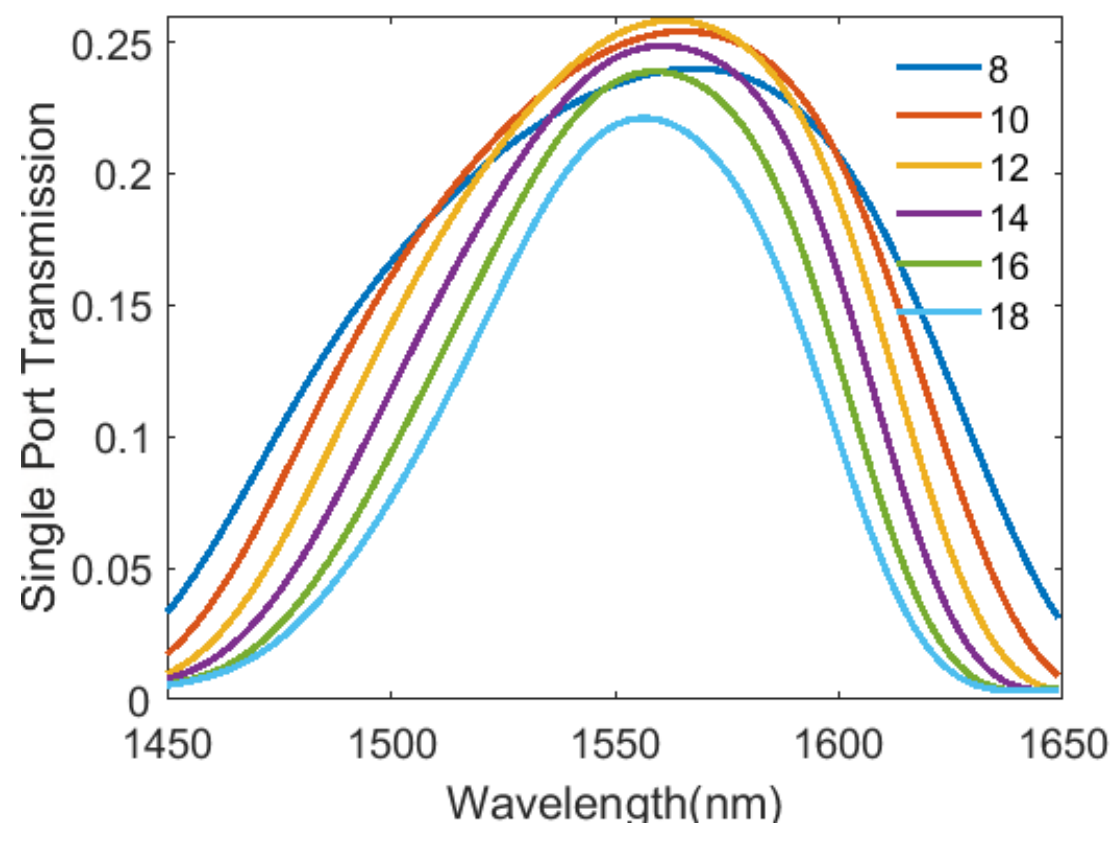

Figure 3.14 Transmission spectrum of the vertical incident grating coupler with a sweep of the number of periods

Figure 3.14 shows that the grating coupler has a maximum coupling efficiency with 10 to 13 periods, but not 15 periods (which is closer to the fiber core diameter). Because a 
fundamental polarization mode has a Gaussian-like shaped power profile, it will concentrate about $70 \%$ of the power in one-third of the mode width range. In this design, a balance between the coupled power and back reflection loss in the waveguide needs to be considered: a short grating length would have enough light to be coupled, and a long grating length may have a strong loss due to the second order back reflection inside the grating structure.

\section{Optimization}

For a vertical incident grating coupler, there are not a lot of parameters that can be used for optimization. The etch depth and number of periods are the two main parameters that need to be optimized. Fill-factor should also be considered carefully.

Table 3.3 Optimized vertical incident grating coupler parameters

\begin{tabular}{|c|c|c|c|c|c|}
\hline$\lambda_{\mathbf{0}}$ & $\boldsymbol{\Lambda}$ & $\mathbf{f f}$ & $\boldsymbol{e d}$ & $\boldsymbol{h}$ & $\mathbf{N}$ \\
\hline $1550 \mathrm{~nm}$ & $578.376 \mathrm{~nm}$ & 0.485 & $73 \mathrm{~nm}$ & $220 \mathrm{~nm}$ & 14 \\
\hline $1310 \mathrm{~nm}$ & $461.554 \mathrm{~nm}$ & 0.485 & $61 \mathrm{~nm}$ & $220 \mathrm{~nm}$ & 17 \\
\hline
\end{tabular}

In table 3.3, the optimized parameters of the vertical incident grating couplers designed for $1550 \mathrm{~nm}$ and $1310 \mathrm{~nm}$ have been listed. Compared with the initial condition values, the optimized design has a close period length and fill-factor, but the etched depth decreased a lot. Different etched depths are used for two different wavelength bands to maximize the transmission. 


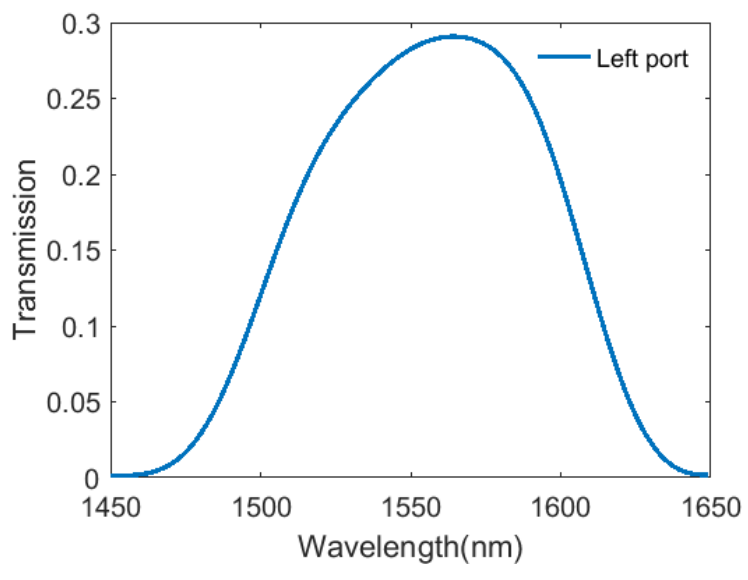

(a)

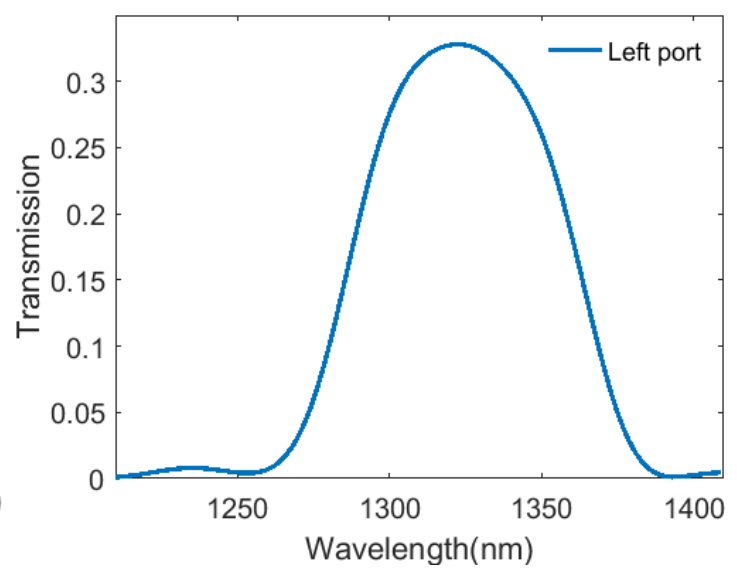

(b)

Figure 3.15 Transmission spectrum of optimized vertical incident grating couplers design for (a) $1550 \mathrm{~nm}$ and (b) $1310 \mathrm{~nm}$

Figure 3.15 shows the FDTD simulation results for the optimized design. The maximum transmission for a single port has increased by around $1.5 \mathrm{~dB}$. The total transmission is $-2.3 \mathrm{~dB}$ for $1550 \mathrm{~nm}$ and $-1.8 \mathrm{~dB}$ for $1310 \mathrm{~nm}$. They have a $3-\mathrm{dB}$ bandwidth of $\sim 90 \mathrm{~nm}$ and $1-\mathrm{dB}$ bandwidth of $\sim 50 \mathrm{~nm}$.

\subsection{Combining of Two Wavelength Designs Grating Couplers}

State-of-the-art dual-wavelength vertical incident grating couplers with nonuniform grating structures have the issue of either having low coupling efficiencies or a small feature size that is difficult to fabricate.

A simple but novel structure of the dual-wavelength vertical incident grating coupler is shown below. It is a combination of the vertical incident grating coupler designed for $1310 \mathrm{~nm}$ and $1550 \mathrm{~nm}$. 


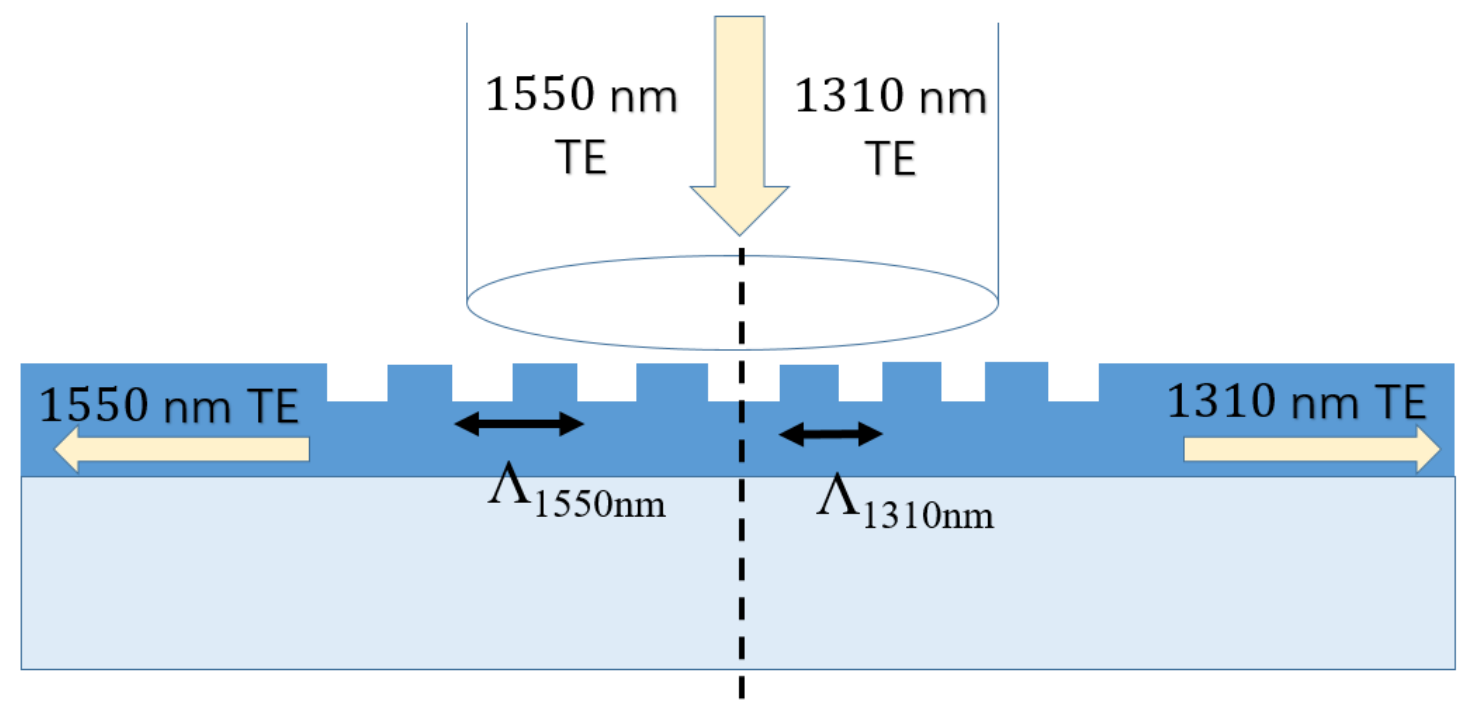

Figure 3.16 Schematic diagram of the dual-wavelength vertical incident grating coupler

In figure 3.16, the grating structure of the dual-wavelength vertical incident grating coupler design has been divided into two parts: the left part with a longer period length is designed for $1550 \mathrm{~nm}$, and the right part with a shorter period length is designed for 1310 $\mathrm{nm}$. The initial condition of this structure is taken directly from the optimized dualwavelength vertical incident grating couplers designed for O-band and C-band in the previous section.

In this structure, because only half of the grating structure is designed for one wavelength, there will be only half of the power incident on the grating structure. As a result, he transmission would have a maximum 50\% limit. Considering the coupling efficiency and loss of the vertical incident grating coupler, a total transmission of around $25 \%(-6 \mathrm{~dB})$ would be observed. 


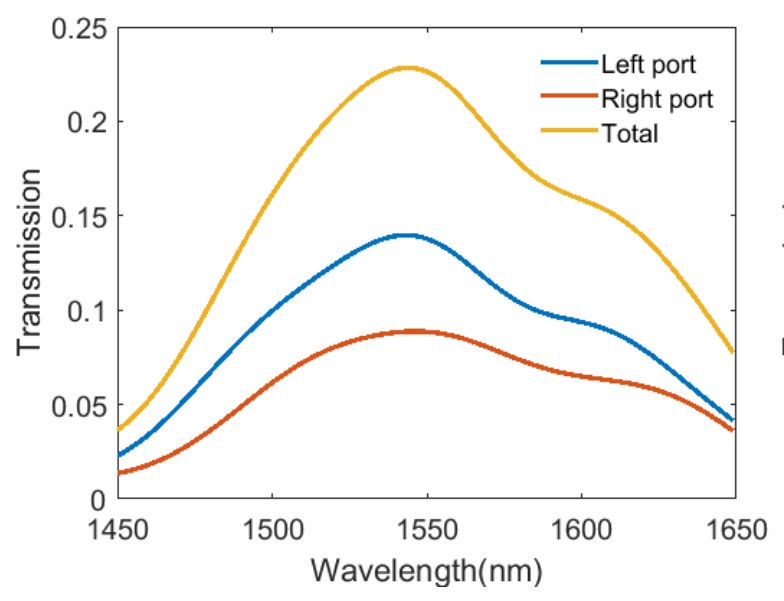

(a)

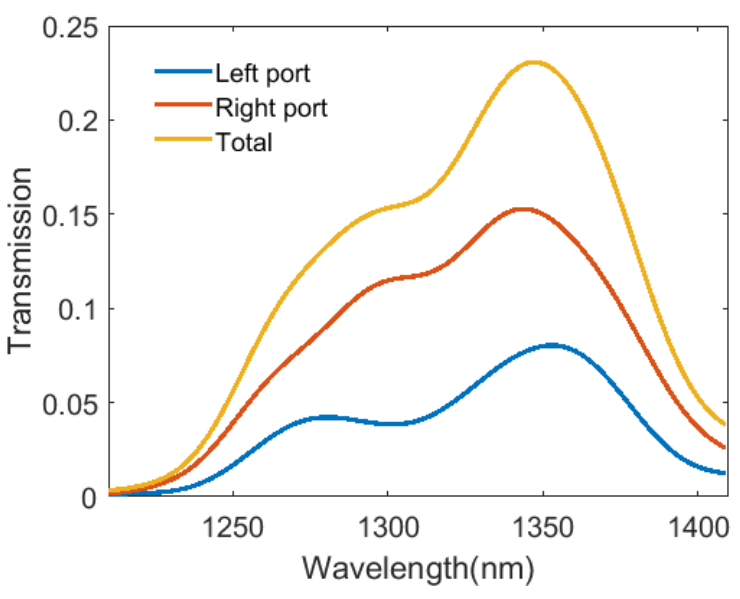

(b)

Figure 3.17 Transmission spectrum of the dual-band vertical incident grating couplers at (a) $1550 \mathbf{~ n m}$ and (b) $1310 \mathrm{~nm}$

From figure 3.17 , maximum coupling efficiencies are close to what would be expected. Both the $1310 \mathrm{~nm}$ and $1550 \mathrm{~nm}$ have a transmission around 23\% (-6.4 dB).

Due to the fiber position is not being at the center of the waveguide grating structure designed for a single wavelength, the left and right ports have different transmission spectrums. In the meantime, from the parameter sweep result of the fiber position in figure 3.9, the left port should have a lower transmission than the right port for $1550 \mathrm{~nm}$ and a higher transmission than the right port for $1310 \mathrm{~nm}$. However, the result shows the opposite because the grating structure design for one wavelength band would also behave as a grating coupler with an incident angle for another wavelength band. The power coupled back to the cladding becomes the loss of the incident light.

One important parameter in this design is the combined width between the two different grating designs. The fiber position would also affect the performance of the grating coupler. 


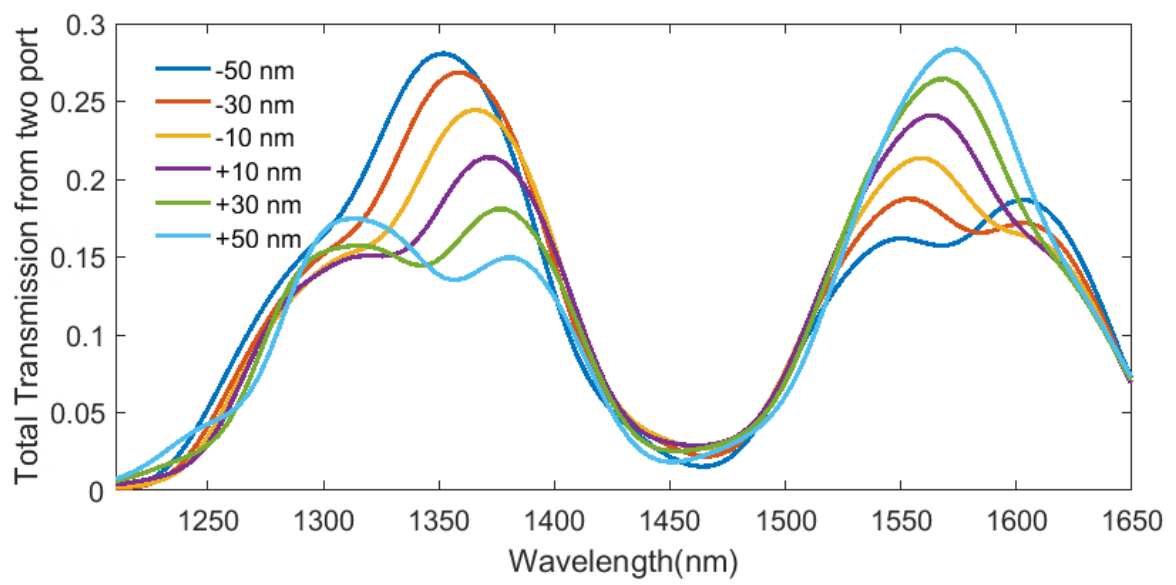

(a)

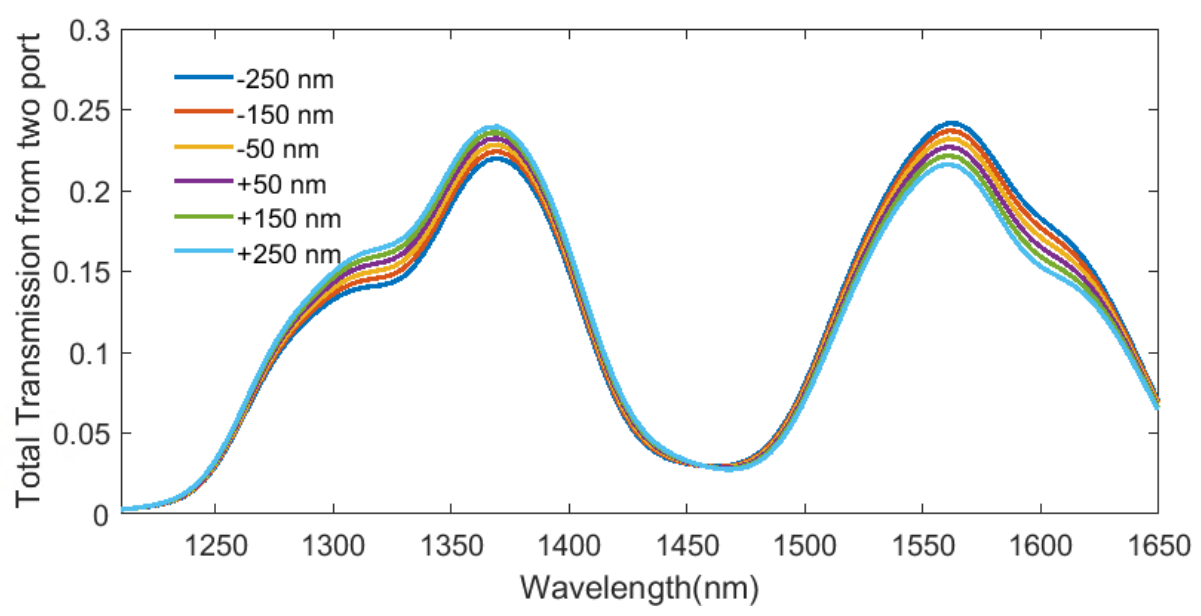

(b)

Figure 3.18 Transmission spectrums of vertical incident grating couplers design with (a) a combined width sweep and (b) a fiber position sweep.

The fiber position has almost no effect on the transmission performance of the grating coupler, as figure 3.18 (b) shows. However, the combined width would affect the transmission a lot. The initial combined width is the average width between the fill-width of $1550 \mathrm{~nm}$ and $1310 \mathrm{~nm}$. While a longer combined width would have a diffraction effect close to the design period length of $1550 \mathrm{~nm}$, a shorter combined width is close to the fillwidth of $1310 \mathrm{~nm}$ light. Under those conditions, coupling efficiencies are difficult to increase for both wavelengths. 


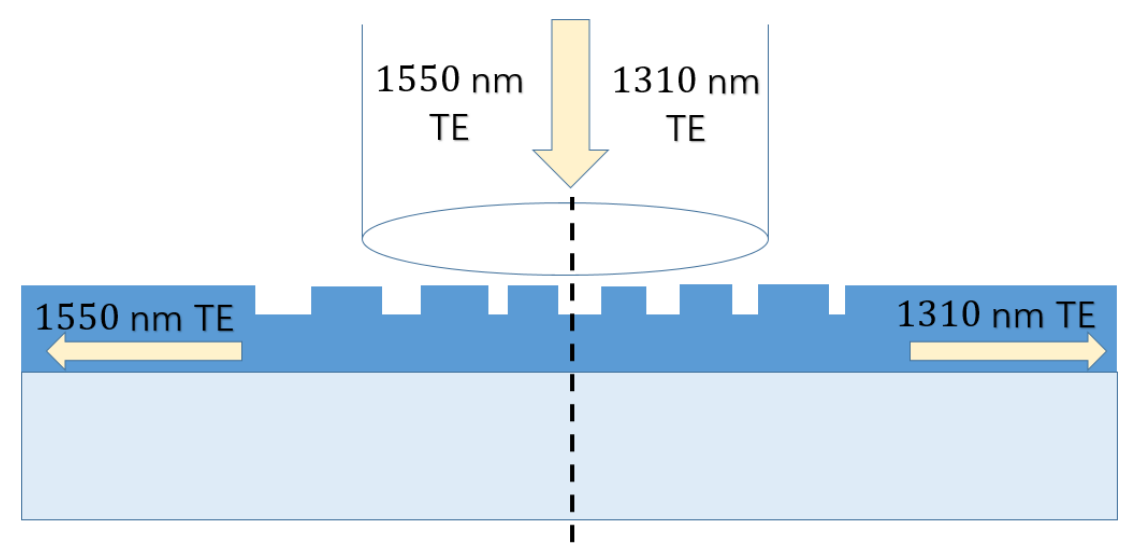

Figure 3.19 Schematic diagram of the apodized dual-wavelength vertical incident grating coupler

An apodized structure is applied to optimize this 2D grating coupler. Because the average effective index would change with the fill-factor, the back reflection would be reduced by applying the apodized structure. A smooth effective index change between two different grating structures would cause less loss. An apodized design example is shown in figure 3.19. Its FDTD simulation result is shown in figure 3.20.

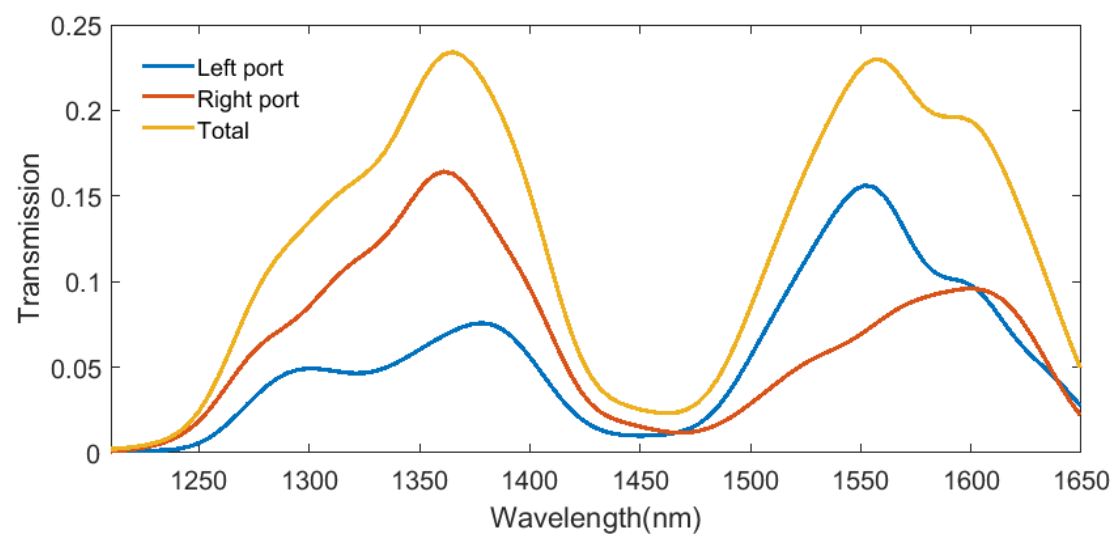

Figure 3. 20 Transmission spectrum of the apodized dual-wavelength vertical incident grating coupler

The total transmission of the apodized design did not increase by much. However, the $1550 \mathrm{~nm}$ at the left port and the $1310 \mathrm{~nm}$ at the right port have transmissions of $\sim 17 \%$ $(-7.7 \mathrm{~dB})$. Transmissions at another port at the same wavelength are around $7 \%$. After apodizing, this vertical incident grating coupler behaves as a wavelength splitter. The total 
transmissions are acceptable when compared with the dual-band vertical incident grating coupler that others had previously designed.

Although the total coupling efficiency is acceptable, the vertical incident grating coupler has strong crosstalk of around $-3 \mathrm{~dB}$ between two bands and cannot split $1550 \mathrm{~nm}$ and $1310 \mathrm{~nm}$ light perfectly. A thick SOI platform with a $340 \mathrm{~nm}$ thickness is used to improve the performance of the vertical incident grating coupler. Because a thicker waveguide would have a lower effective index difference between $1550 \mathrm{~nm}$ and $1310 \mathrm{~nm}$, gratings designed for one wavelength can be used as a chip-to-fiber grating coupler for another wavelength to reduce crosstalk. Figure 3.21 shows the transmission spectrum of a 340-nm-thick apodized vertical incident grating coupler.

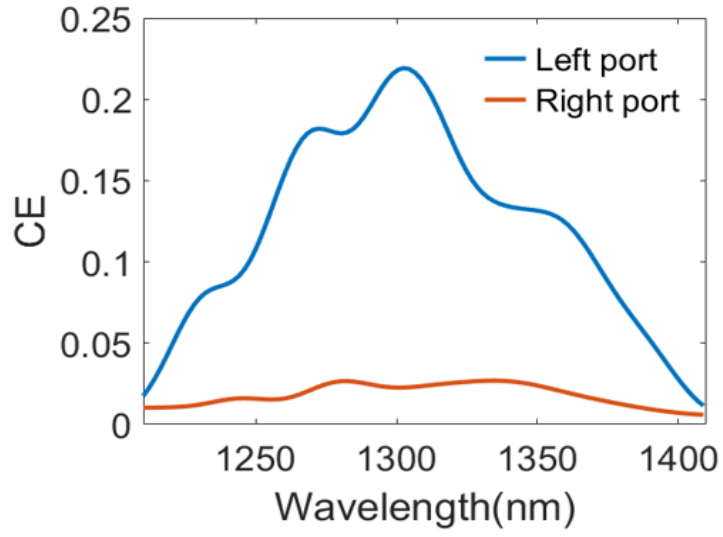

(a)

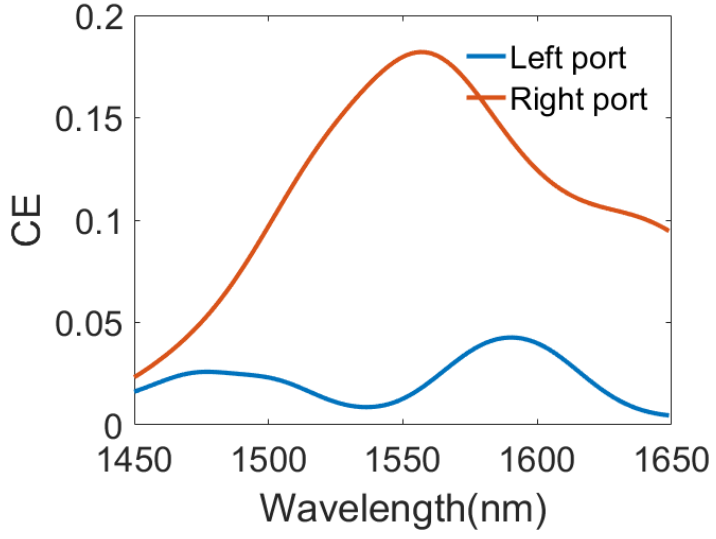

(b)

Figure 3.21 Coupling efficiency spectrum of the optimized dual-wavelength vertical incident grating coupler at (a) $1310 \mathrm{~nm}$ and (b) $1550 \mathrm{~nm}$

The maximum transmissions of the two bands are around $20 \%$ in figure 3.21 . Compared with the 220-nm-thick designs shown previously, this design has low crosstalk between two output ports. This design is a feasible dual-band vertical incident grating coupler for wavelength demultiplexing. 


\section{Chapter 4:}

\section{Dual-band Polarization Splitting Grating}

\section{Coupler}

With increasing demand for a higher speed communication system such as the wavelength-division-multiplexing (WDM) systems, both polarization independent and dual-band on-chip grating couplers are required. The polarization independent or polarization splitting grating coupler can be used to increase the maximum data capacity or to simplify the coupling processing. An on-chip dual-band (C- band and $\mathrm{O}$-band) grating coupler can work as a low-cost transceiver in fiber-to-the-home network (FTTH) systems. All the grating couplers developed for the WDM communication system are either dualwavelength or polarization independent with a board bandwidth. A grating coupler that can work for two wavelength-bands with two polarizations has not been developed.

In this chapter, a design flow for a wavelength and polarization splitting grating coupler will be presented. First, this chapter will begin with the design for a polarization independent grating coupler. Afterward, the 2-D grating coupler design with square-shaped holes will be introduced and used for realizing wavelength and polarization splitting at the same time. 


\subsection{Polarization Splitting Grating Coupler Design}

To design a polarization splitting or polarization independent grating coupler, the average effective index of the wave propagating in the waveguide needs to satisfy Braggs condition for TE and TM polarization at the same time. Under this condition, because the operating wavelength and period length are known for a given grating structure, the Bragg condition can be written as:

$$
n_{\text {aveeffTE }}-n_{\text {SiO }_{2}} \sin ( \pm \theta)=\frac{\lambda_{0}}{\Lambda}=n_{\text {aveeffTM }}-n_{\text {SiO }_{2}} \sin ( \pm \theta)
$$

By simplifying equation 4.1, the relationship between the average effective index of TE and TM mode can be written as:

$$
n_{\text {aveeffTE }} \pm n_{\text {SiO }_{2}} \sin (\theta)=n_{\text {aveeffTM }} \pm n_{S i O_{2}} \sin (\theta)
$$

So that a polarization independent grating coupler will have an average TE effective index that equals to average TM effective index $\left(n_{\text {aveeffT }}=n_{\text {aveeffTM }}\right)$. In the meantime, the polarization splitting grating coupler would satisfy:

$$
n_{\text {aveeffTE }}-n_{\text {aveeffTM }}=2 \cdot n_{\text {SiO }_{2}} \sin (\theta)
$$

There is no plus-minus sign at the right side of equation 4.3 because the effective index of the TE polarization is always larger than the TM polarization for any thickness's grating waveguide structure. Obviously, it is challenging to realize polarization independence for a conventional grating coupler structure. In this chapter, the polarization splitting grating coupler design flow will be presented.

Similar to figure 3.2, with an initial condition, a figure of Bragg condition with the absolute value of the incident angle $(|\theta|)$ versus the period length $(\Lambda)$ can be plotted as figure 4.1 shows. 


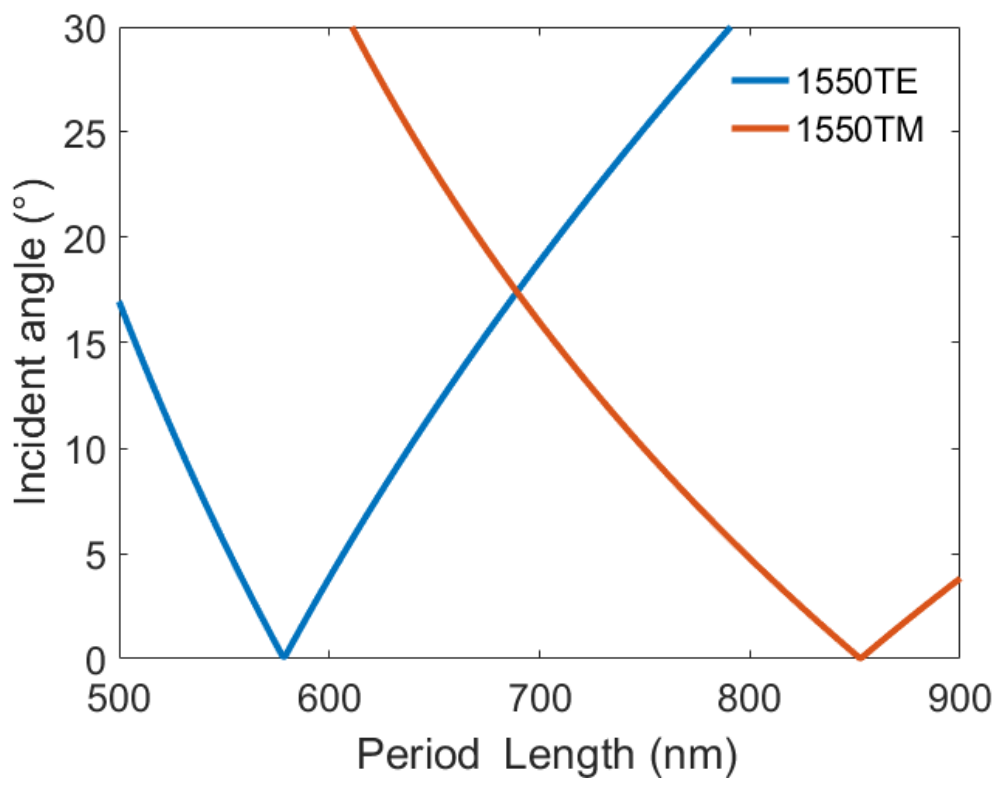

Figure 4.1 The absolute value of the incident angle versus the period length based on the Bragg condition

By using the absolute value of the incident angle $(|\theta|)$, a cross point of two lines of polarization can be observed. In figure 4.1, while a descent line means a negative incident angle that satisfied the Bragg condition, a rising line indicates a positive incident angle. The cross point of those two lines shows the parameters where the grating coupler can work as a polarization splitter: the TE mode has a backward coupling, and the TM mode is a forward coupling. This relationship can also be shown in a schematic Bragg condition diagram, as in figure 4.2. There will be no second-order diffraction for the 1550 $\mathrm{nm}$ in this waveguide structure.

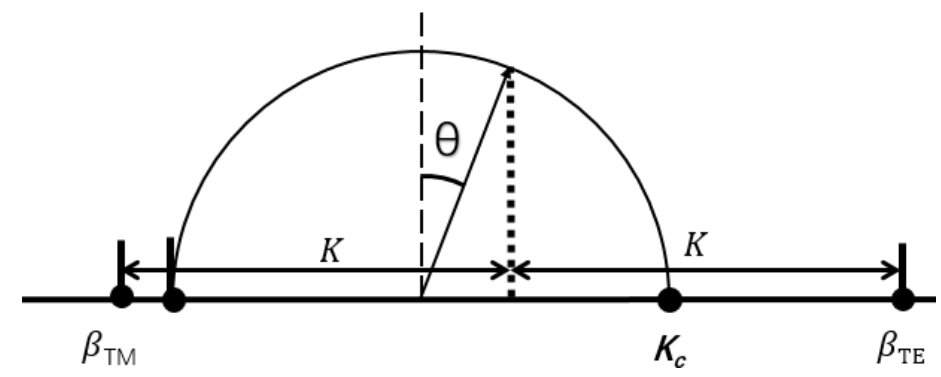

Figure 4.2 The schematic Bragg condition for a polarization splitting grating coupler 


\subsection{Dual-Band Polarization Splitting Grating Coupler}

If we want to realize a polarization splitting design for both $1550 \mathrm{~nm}$ and $1310 \mathrm{~nm}$ at the same time in a straight waveguide, the average effective indexes of $1550 \mathrm{~nm}$ and $1310 \mathrm{~nm}$ must be equal. However, for any thickness of silicon waveguide, the effective index of $1550 \mathrm{~nm}$ is smaller than $1310 \mathrm{~nm}$ for both TE and TM polarizations. This result is shown in figure 3.1. Under this condition, a grating coupler with a square-shaped-hole structure would be applied.

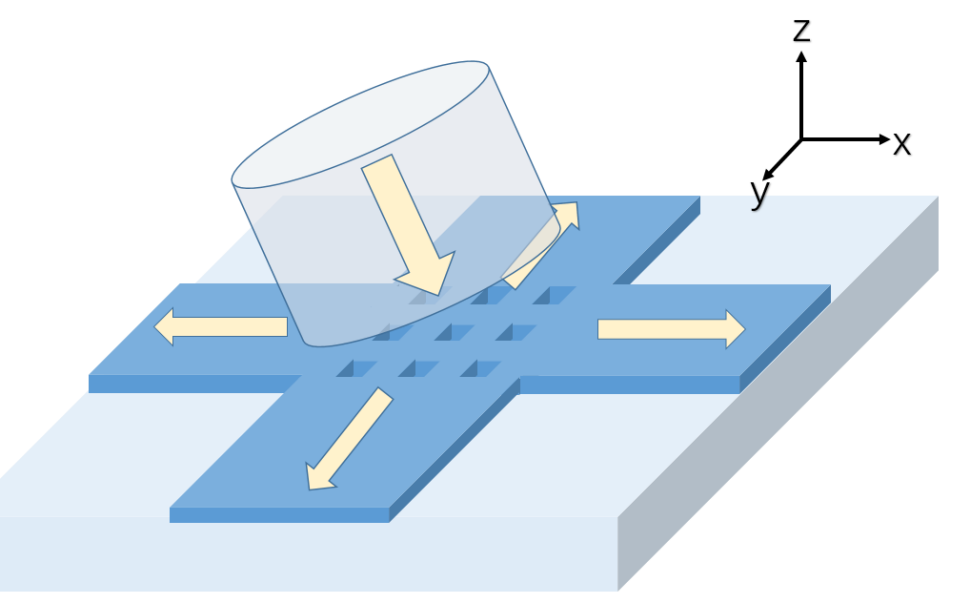

(a)

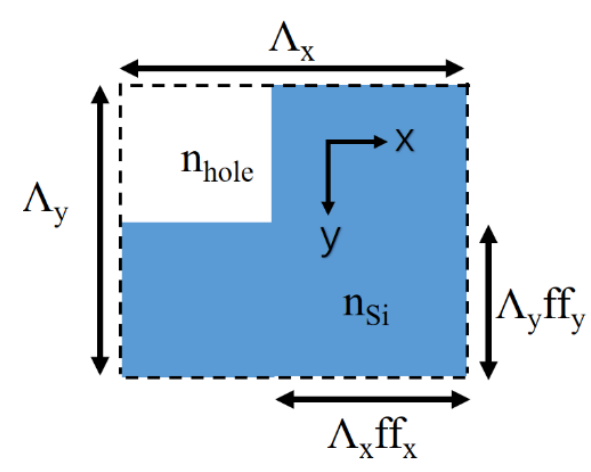

(b)

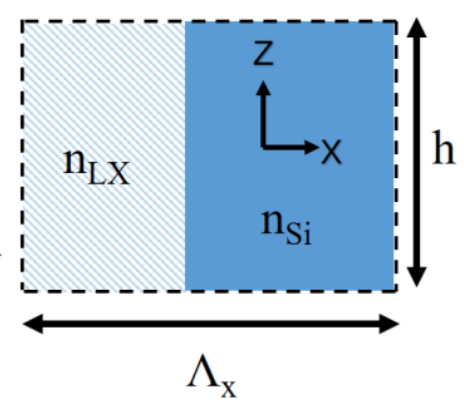

(c)

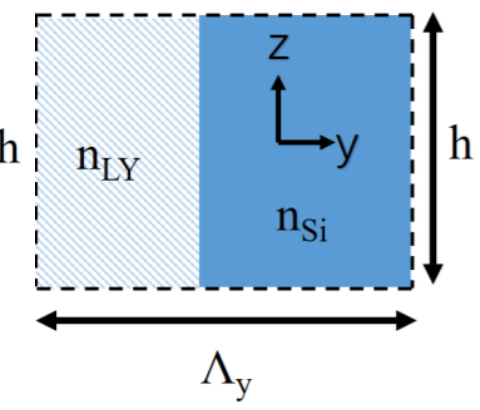

(d)

Figure 4.3 the schematic diagrams of (a) four ports grating coupler and its (b) top view, (c) side view from $x z$ plane and (d) size view from yz plane for one period 
This structure is a cross-shaped grating coupler which has four output ports. It is a combination of two orthogonal straight grating couplers. In this structure, the etched grating in the 1-D grating structure is replaced by a square-shaped hole structure. This structure is periodical in both the $\mathrm{x}$ - and y-axis. Period lengths are named as $\Lambda_{x}$ and $\Lambda_{y}$, fill-factors are $\mathrm{ff}_{x}$ and $\mathrm{ff}_{y}$, and $\mathrm{n}_{\mathrm{Lx}}$ and $\mathrm{n}_{\mathrm{Ly}}$ provide the equivalent reflective index of the square-shaped-hole structure. In this structure, the effective index of the etched part needs to be recalculated by equation 2.19. So that the average effective index calculation equation (equation 3.2) would rewrite as:

$$
\begin{aligned}
& n_{\text {aveeff }}=n_{\text {effSi }} \cdot \mathrm{ff}_{x}+\left(1-\mathrm{ff}_{x}\right) /\left(\mathrm{ff}_{y} \cdot n_{\text {hole }^{-1}}+\mathrm{ff}_{y} \cdot n_{\text {effSi }^{-1}}\right) \\
& n_{\text {aveeff }}=n_{\text {effSi }} \cdot \mathrm{ff}_{y}+\left(1-\mathrm{ff}_{y}\right) /\left(\mathrm{ff}_{x} \cdot n_{\text {hole }^{-1}}+\mathrm{ff}_{x} \cdot n_{\text {effSi }^{-1}}\right)
\end{aligned}
$$

For this structure, the effective index can be designed individually for the $\mathrm{x}$ - and $\mathrm{y}$ axis to achieve polarization splitting for two wavelengths. However, the period length designed for the $\mathrm{x}$ - and $\mathrm{y}$-axis must be larger than $500 \mathrm{~nm}$ because a shorter period length might have a subwavelength effect rather than the Bragg diffraction. The subwavelength structure will follow a zeroth- or second-order EMT approximation.

Usually, the square-shaped holes are fully etched. The fully etched gratings have advantages in fabrication with drilling because the etched depth does not need to be considered. A circular-shaped-hole structure was also used sometimes instead of the square-shaped holes. The circular-shaped-hole has a grating performance almost the same as the square-shaped holes.

Figure 4.4 shows the incident angle versus the grating period length for both polarizations of $1310 \mathrm{~nm}$ and $1550 \mathrm{~nm}$. It is calculated based on an initial condition for a $220 \mathrm{~nm}$ SOI platform with 0.5 fill-factor for both the $\mathrm{x}$ - and $\mathrm{y}$-axis. 


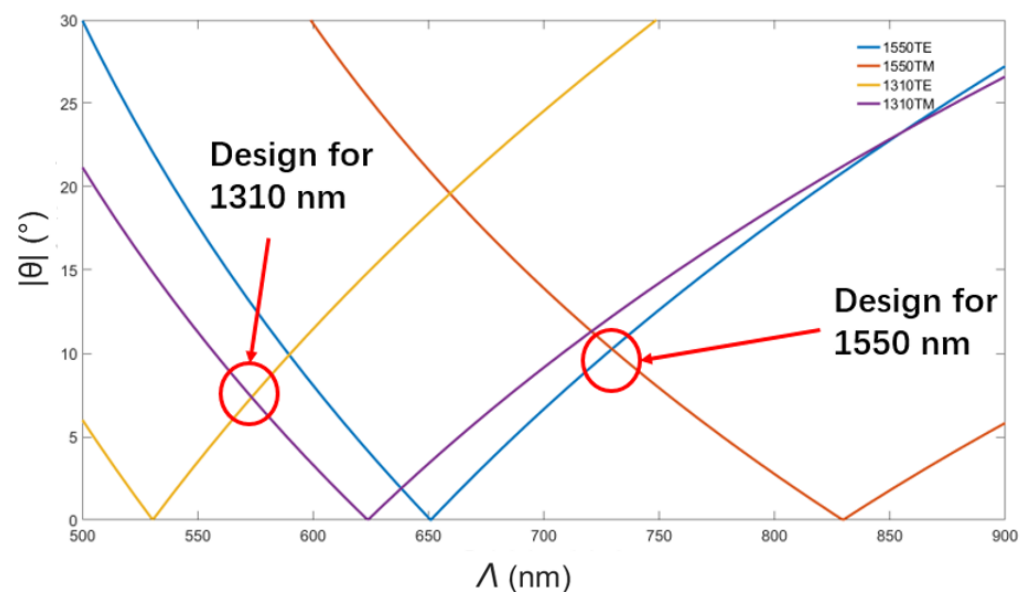

Figure 4.4 the schematic diagrams of (a) four ports grating coupler and its (b) top view, (c) side view from xz plane and (d) size view from yz plane

In figure 4.4 , because of the fill-factors $\mathrm{ff}_{x}$ and $\mathrm{ff}_{y}$ have the same value, equation 4.4 and 4.5 are the same too. Any cross point in the figure can be used as the design parameters of the four output gratings. There is one unique cross point of two increasing lines (1550 nm TE mode and $1310 \mathrm{TM}$ mode) at the period length of $850 \mathrm{~nm}$ and the incident angle of $25^{\circ}$. With those design parameters, $1550 \mathrm{~nm}$ TE mode and $1310 \mathrm{TM}$ mode can couple into the same direction waveguide. Because the line of $1550 \mathrm{~nm}$ TE is close to $1310 \mathrm{~nm}$ TM, they would have a strong cross talk and be very lossy.

As this chapter explains, the four-port grating coupler is designed as a dualwavelength polarization splitting grating coupler. It has an $\mathrm{x}$-axis designed for $1550 \mathrm{~nm}$ and a y-axis designed for $1310 \mathrm{~nm}$. The design parameters are listed in table 4.1, and the design diagram is shown in figure 4.5 .

Table 4.1 Design parameters for the four ports grating coupler

\begin{tabular}{|c|c|c|c|c|}
\hline & $\boldsymbol{\lambda}_{\mathbf{0}}$ & $\mathbf{f f}$ & $\boldsymbol{\Lambda}$ & $\boldsymbol{\theta}$ \\
\hline x-axis & $1550 \mathrm{~nm}$ & 0.5 & $729.55 \mathrm{~nm}$ & $10.259^{\circ}$ \\
\hline y-axis & $1310 \mathrm{~nm}$ & 0.5 & $573.31 \mathrm{~nm}$ & $7.366^{\circ}$ \\
\hline
\end{tabular}




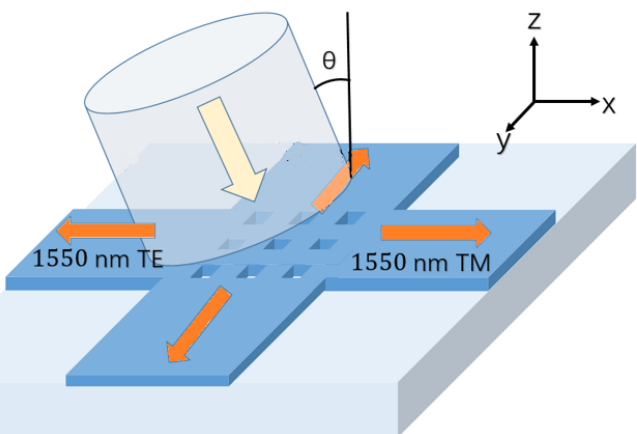

(a)

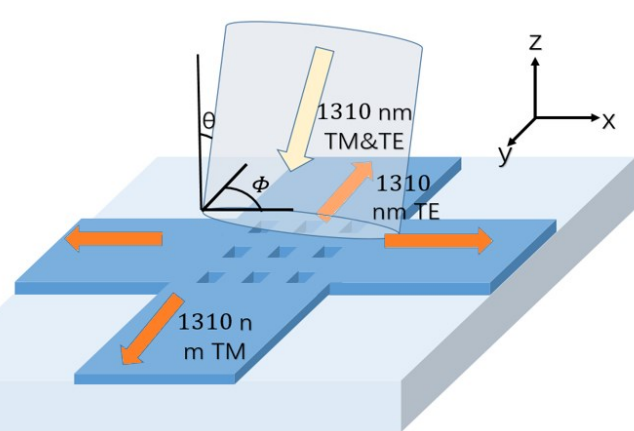

(b)

Figure 4.5 The schematic diagram for the four ports grating coupler that works for (a) $1550 \mathrm{~nm}$ and (b) $1310 \mathrm{~nm}$.

By modeling and setting the grating structure with the design parameters in the 3D FDTD simulation, the transmission spectrum result is shown in figure 4.6.

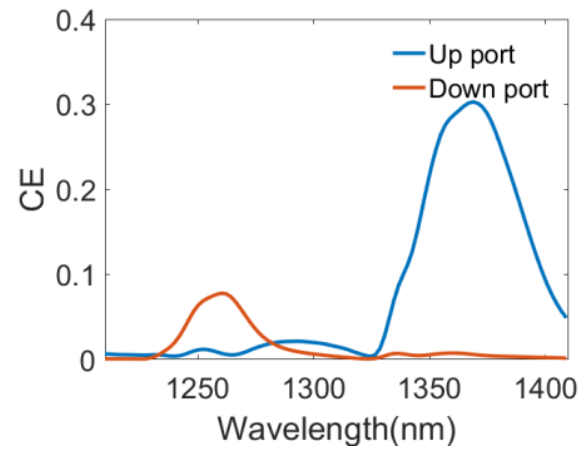

(a)

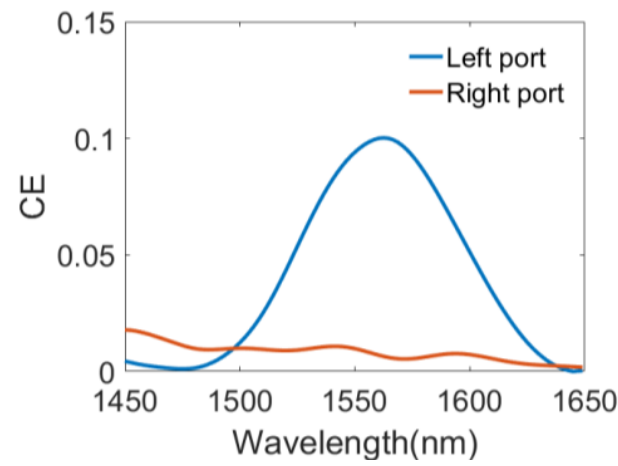

(c)

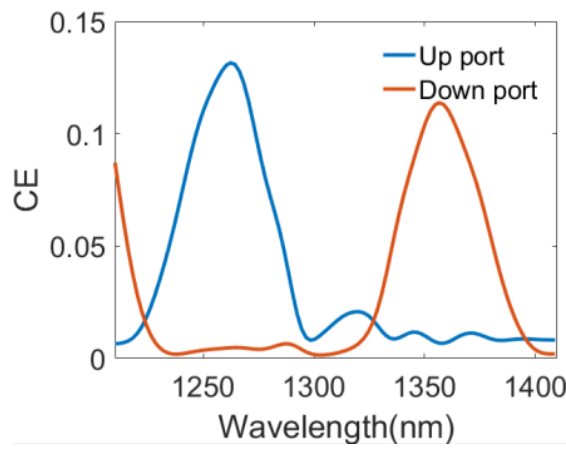

(b)

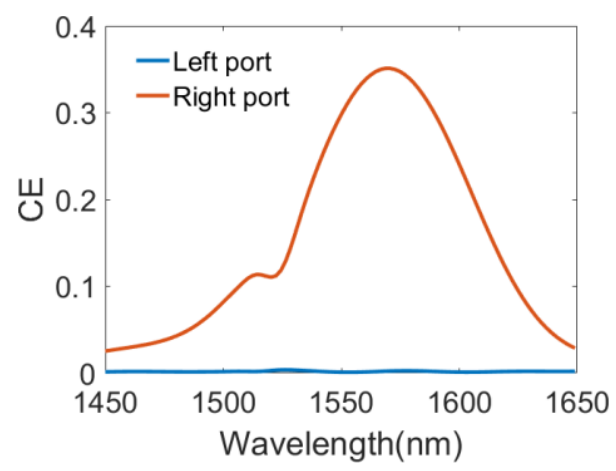

(d)

Figure 4.6 The transmission spectrum of four ports grating coupler with (a) $1310 \mathrm{~nm}$ TE, (b) 1310 nm TM, (c) $1550 \mathrm{~nm}$ TE, (d) $1550 \mathrm{~nm}$ TM 
As figure 4.6 shows, this grating coupler has a center wavelength at $1570 \mathrm{~nm}$ and $1360 \mathrm{~nm}$. Because a $573.31 \mathrm{~nm}$ period length is close to the subwavelength limit (about $450 \mathrm{~nm})$, the simulation result will not match the calculation result perfectly. The $1310 \mathrm{~nm}$ TM and $1550 \mathrm{~nm}$ TE have a low coupling efficiency of around 10\%. Because of the strong crosstalk between these four output ports, this kind of structure might lead to strong loss. In the meantime, this design has a major constraint is that it needs an addition angle adjustment to realize the polarization splitting for two wavelengths. 


\section{Chapter 5:}

\section{Dual-Wavelength Polarization}

\section{Independent Grating Coupler}

The two-dimensional periodical grating coupler has its limit about wavelength and polarization insensitivity. A non-uniform design would mainly rely on machine learning, while the subwavelength structure has a minimum feature size limit. A two-dimensional structure with square-shape or circular-shape gratings is introduced in the previous chapter. Although it can support two wavelength-bands and split TE and TM polarizations, the additional angle adjusting is required to realize the polarization splitting for both wavelengths. In this chapter, the design of novel dual-band polarization independent subwavelength grating couplers for wavelength demultiplexing will present. This design does not need to do any angle operating and can support two polarizations for both O-band and C-band at the same time. At the beginning of this chapter, the square-shape holes with a subwavelength feature size will be introduced. The polarization independent design realized by the subwavelength structure would show next. At the end of this chapter, the performance of the novel design will be presented.

\subsection{Two-Dimensional Subwavelength Polarization Independent Grating Coupler}

As the explanation at the beginning of chapter four, it is almost impossible to realize polarization independent on a conventional grating coupler. A subwavelength squareshape-hole structure is used to solve this problem. 


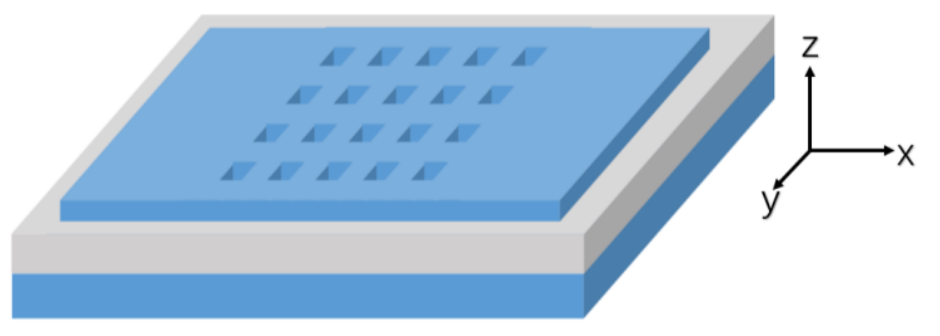

(a)

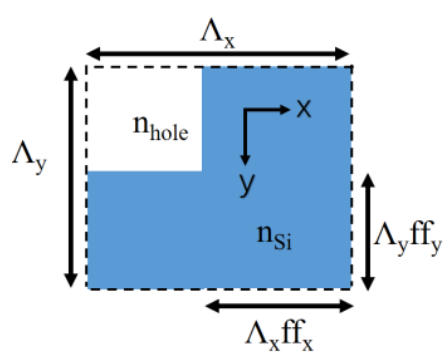

(b)

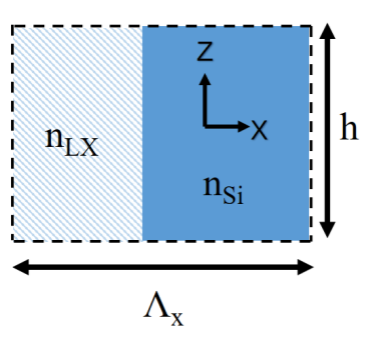

(c)

Figure 5.1 the schematic diagrams of (a) a grating coupler with subwavelength square-hole structure and its (b) top view and (c) side view for one period

Zeroth- and second-order EMT approximation would apply to the subwavelength structure. For the square-shape-hole structure shown in figure 5.1, the EMT zeroth- and second-order approximation presented in chapter 2 would rewrite as the following.

$$
\begin{gathered}
\frac{1}{n_{\mathrm{LTE}}^{(0)}}=\sqrt{\left[\frac{\mathrm{ff}_{y}}{n_{\text {hole }}^{2}}+\frac{\left(1-\mathrm{ff}_{y}\right)}{n_{\mathrm{Si}}^{2}}\right]} \\
\frac{1}{n_{\mathrm{LTM}}^{(0)}}=\sqrt{\left[n_{\text {hole }^{2}} \mathrm{ff}_{y}+n_{\mathrm{Si}}^{2}\left(1-\mathrm{ff}_{y}\right)\right]} \\
\mathrm{n}_{\mathrm{LTE}}^{(2)}=\mathrm{n}_{\mathrm{LTE}}^{(0)} \sqrt{\left[1+\frac{\pi^{2}}{3}\left(\frac{\lambda_{0}}{n_{\mathrm{effHTE}}}\right)^{2} \mathrm{ff}_{y}{ }^{2}\left(1-\mathrm{ff}_{y}\right)^{2}\right]} \\
\times \sqrt{\left(\mathrm{n}_{\text {hole }}^{2}-\mathrm{n}_{\mathrm{Si}}^{2}\right)^{2}\left(\frac{\mathrm{n}_{\mathrm{LTM}}^{(0)}}{n_{\text {effTE }}}\right)^{2}\left(\frac{\mathrm{n}_{\mathrm{LTE}}^{(0)}}{n_{\text {hole }} n_{\mathrm{Si}}}\right)^{4}}
\end{gathered}
$$




$$
\mathrm{n}_{\mathrm{LTM}}^{(2)}=\mathrm{n}_{\mathrm{LTM}}^{(0)} \sqrt{\left[1+\frac{\pi^{2}}{3}\left(\frac{\lambda_{0}}{n_{\text {effHTM }}}\right)^{2} \mathrm{ff}_{y}{ }^{2}\left(1-\mathrm{ff}_{y}\right)^{2}\left(\frac{\mathrm{n}_{\mathrm{hole}}^{2}-\mathrm{n}_{\mathrm{Si}}^{2}}{n_{\text {effHTM }} \mathrm{n}_{\mathrm{LTM}}^{(0)}}\right)^{2}\right.}
$$

Where $\mathrm{n}_{\mathrm{LTM}}^{(0)}, \mathrm{n}_{\mathrm{LTM}}^{(2)}, \mathrm{n}_{\mathrm{LTE}}^{(0)}$ and $\mathrm{n}_{\mathrm{LTE}}^{(2)}$ mean the zeroth- or second-order EMT effective index for TE and TM polarizations. They are the effective indexes of the subwavelength square-shape grating structure and be working as the low effective index part of the gratings. $n_{\text {hole }}$ and $n_{\mathrm{Si}}$ are the refractive index of the hole (which is $\mathrm{SiO}_{2}$ in this design) and silicon, $\mathrm{ff}_{y}$ donates the fill-factor of the y-direction, $n_{\text {effHTM }}$ and $n_{\text {effHTE }}$ represent the effective index of the TE and TM mode of the filling part of the grating and donate the high effective index.

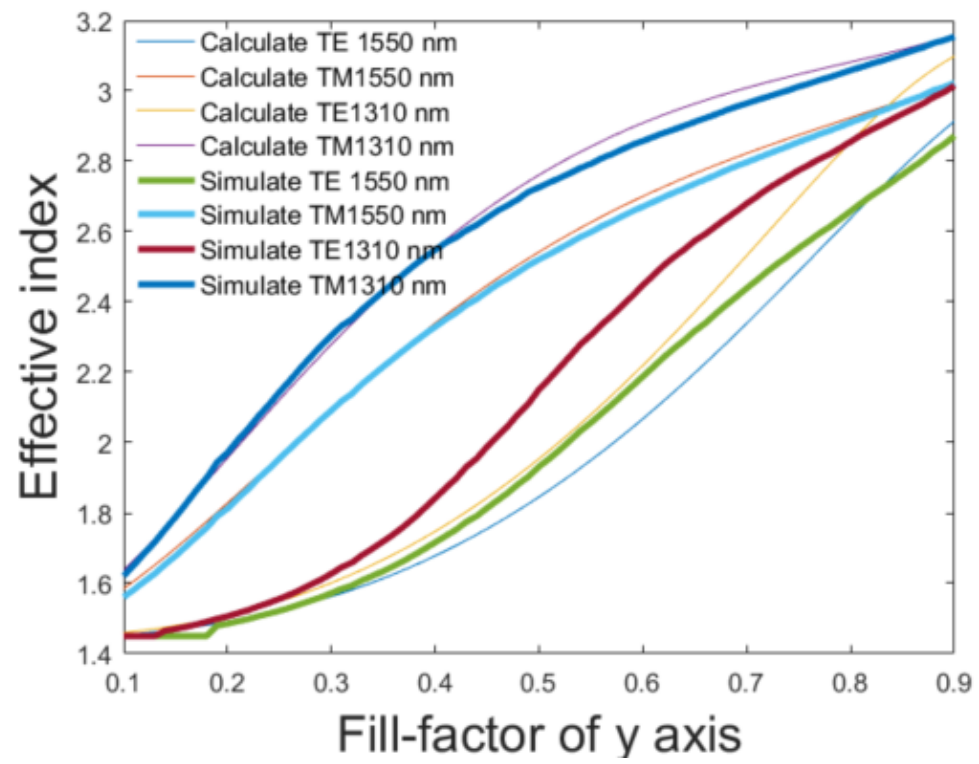

Figure 5.2 Calculation and simulation result of the effective index of the square-shape subwavelength structure.

Because of the minimum feature size limit, the second-order EMT approximation is selected. Its effective index versus $\mathrm{ff}_{y}$ has been plotted in figure 5.2. The calculation result is based on equation 5.3 and 5.4, while the simulation result is getting from Lumerical Mode. The calculation result and simulation result almost agree with each other for TM 
mode and have a maximum $10 \%$ error for the TE mode. Because of the complication of analyzing the effective index of subwavelength structure, this $10 \%$ error is acceptable.

By comparing the effective index of the subwavelength structure shown in figure 5.2 with the effective index versus thickness plot in figure 3.1, it can be found that the effective index of TE mode decreases dramatically. In the meantime, the effective index of TM mode has not changed a lot. For a grating structure using the non-etched waveguide with a high $n_{\text {effTE }}$ and low $n_{\text {effTM }}$ as the filling part and subwavelength square-shape waveguide with a low $n_{\text {effTE }}$ and high $n_{\text {effTM }}$ as the etched part, the average effective indexes of two different polarizations can be the same.

The average effective index calculation for this structure can be written as:

$$
n_{\mathrm{eff}}=n_{\mathrm{effH}} \cdot \mathrm{ff}_{x}+\mathrm{n}_{\mathrm{L}}^{(2)} \cdot\left(1-\mathrm{ff}_{x}\right)
$$

where the $\mathrm{n}_{\mathrm{L}}^{(2)}$ donates the second-order EMT result and $\mathrm{ff}_{x}$ represents the fill-factor of the $\mathrm{x}$-axis.

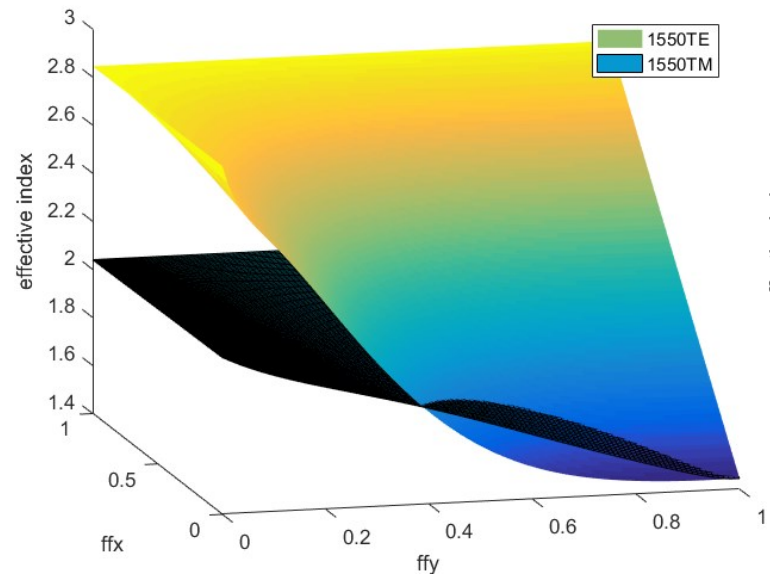

(a)

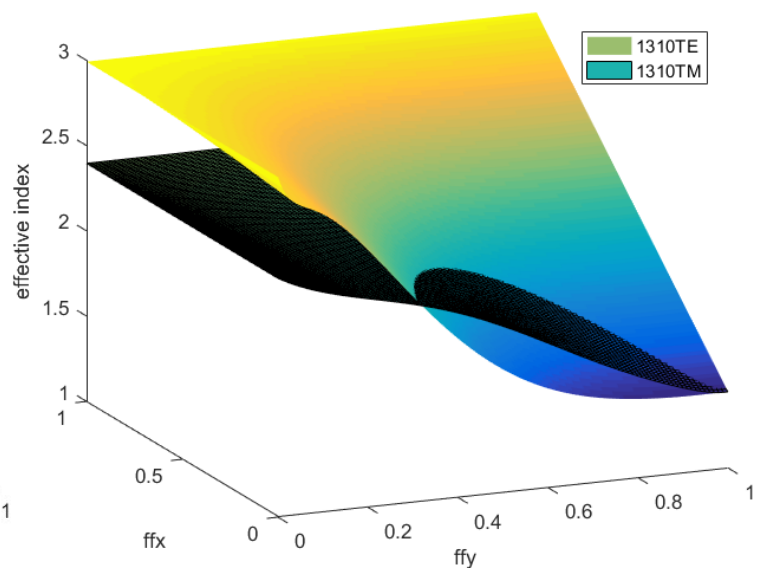

(b)

Figure 5.3 The effective index versus $f f_{\mathrm{x}}$ and $f f_{\mathrm{y}}$ of (a) $1550 \mathrm{~nm}$ and (b) $1310 \mathrm{~nm}$ in a grating coupler with subwavelength square-shape-hole structure. 
With an initial value of the thickness of the grating coupler, the equation of the average effective index written as a function of fill-factors of $\mathrm{x}$ - and $\mathrm{y}$-axis can be expressed from equation 5.3, 5.4, and 5.5, where equation 5.3 and 5.4 are related to $\mathrm{ff}_{y}$ and and equation 5.5 is related to $\mathrm{ff}_{x}$.

Figure 5.3 plotted the average effective index of this square-shaped structure with different fill-factors of $\mathrm{x}$ - and $\mathrm{y}$-axis for a $220 \mathrm{~nm}$ SOI platform in 3-D. Every point on this figure gives an average effective index of the SWGs structure with corresponding $\mathrm{ff}_{x}$ and $\mathrm{ff}_{y}$. The interface of the curved surfaces has the same average effective indexes for both TE and TM modes. By applying the square-shape holes EMT method, for every single fillfactor of $y$-direction, the average effective index of the TE mode can be equal to the average effective index of the TM mode with a particular fill-factor of x-direction.

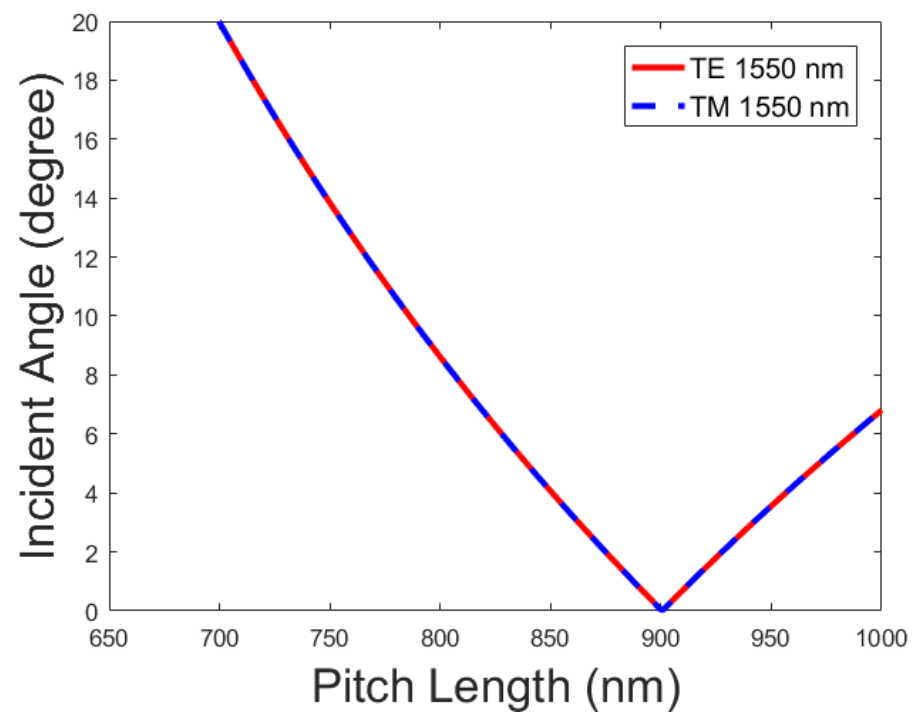

Figure 5.4 The incident angle versus period length for a polarization independent grating coupler with square-shape holes.

By using the fill-factors data on the interfaced line with the effective index, the grating structure with the incident angle and period length can be determined from the Bragg condition. Figure 5.4 shows that the TE polarization and TM polarization were 
overlapped with each other because their average useful indexes are identical. The polarization independent grating coupler can design with any parameters on this plot.

\subsection{Dual Bands Polarization Independent Grating coupler}

For a dual-band polarization independent grating coupler, the polarization independent property makes it much easier to realize the coupling between the optic fiber and the chip. In the meantime, the TE and TM modes can work individually to increase the data rate or bandwidth of the communication system.

For the given thickness grating coupler with a subwavelength square-shape-hole structure, the effective indexes can be calculated for both $1550 \mathrm{~nm}$ and $1310 \mathrm{~nm}$ with both $\mathrm{TE}$ and TM polarization as a function of the $\mathrm{x}-$ and $\mathrm{y}$-axis fill-factors.

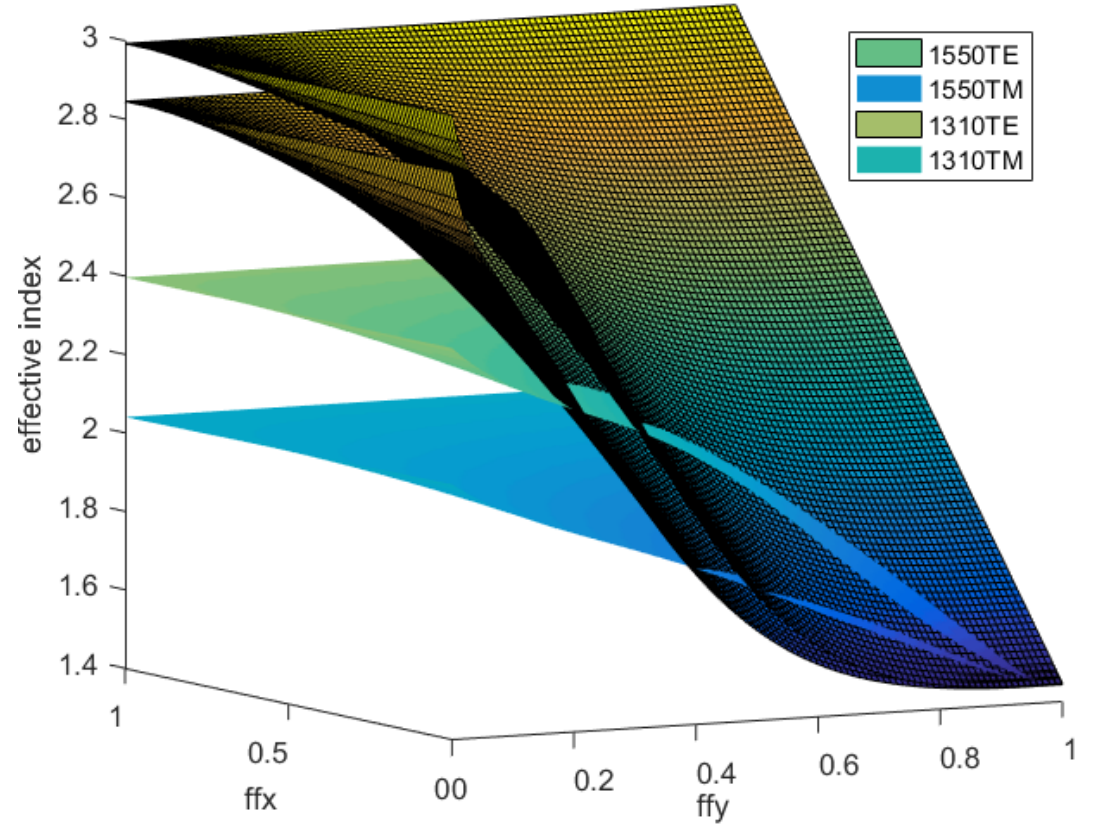

Figure 5.5 The effective index versus $f f_{\mathrm{x}}$ and $f f_{\mathrm{y}}$ of $1550 \mathrm{~nm}$ and $1310 \mathrm{~nm}$ in a grating coupler with subwavelength square-shape-hole structure. 
Figure 5.5 combined figure 5.3 (a) and (b) into one 3-D plot. If there is an existing point that can be a cross point of all four surfaces, this point would have the parameters of $\mathrm{ff}_{x}$ and $\mathrm{ff}_{y}$ that satisfy polarization independent for both $1310 \mathrm{~nm}$ and $1550 \mathrm{~nm}$.

Because the effective index of $1310 \mathrm{~nm}$ light is always larger than the $1550 \mathrm{~nm}$ light in the SOI platform waveguide for any thickness or polarizations or structures as figure 3.1 and 5.2 shown, it is almost impossible to design a polarization independent and wavelength independent grating coupler.

A 2-D $\mathrm{ff}_{x}$ versus $\mathrm{ff}_{y}$ plot can observe the polarization independent design parameters more straightforwardly. The 2-D plot is coming from the 3-D plot (figure 5.3) without the z-axis (average effective index) since the average effective index value will only be used when doing the calculation with Bragg condition.

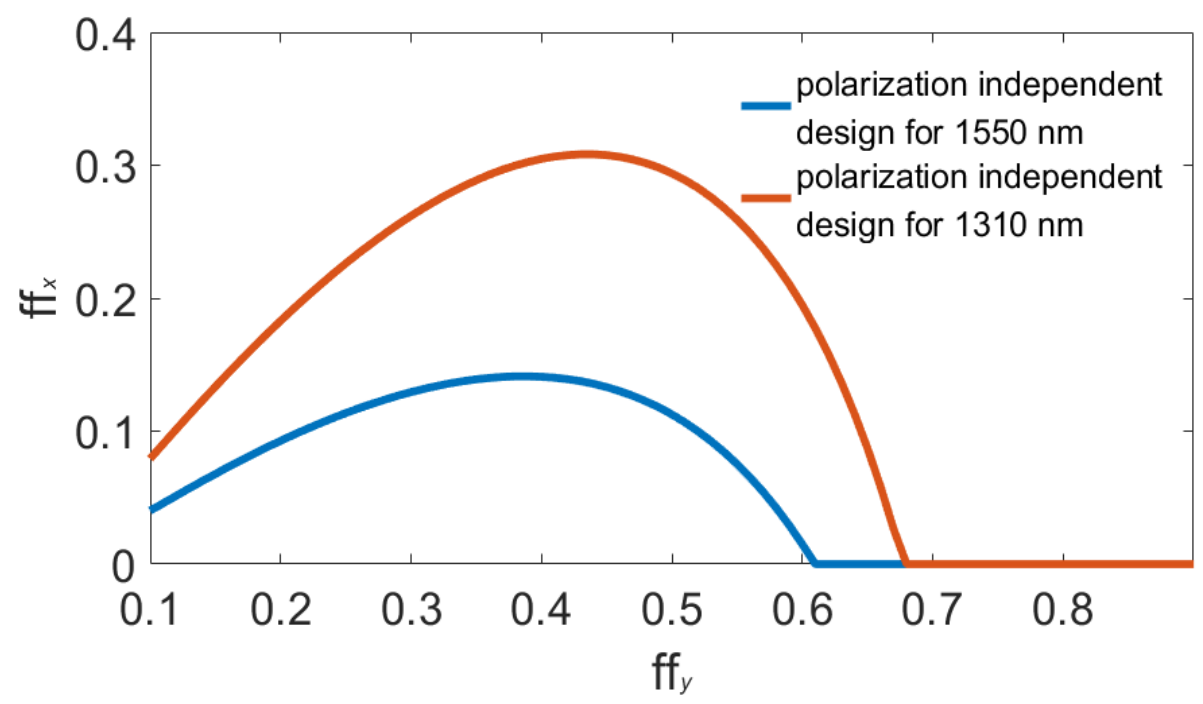

Figure 5.6 The $f f_{\mathrm{x}}$ versus $f f_{\mathrm{y}}$ that satisfy polarization independent for $1550 \mathrm{~nm}$ and $1310 \mathrm{~nm}$ in a grating coupler with subwavelength square-shape-hole structure.

The inherent effective index difference between $1310 \mathrm{~nm}$ and $1550 \mathrm{~nm}$ decided the dual-band polarization independent grating coupler can only be designed as a wavelength demultiplexer. Figure 5.6 shows that there is no cross point between those two polarization 
independent design lines. In the meantime, $1310 \mathrm{~nm}$ is easier to realize polarization independent than $1550 \mathrm{~nm}$ because it has a broader fill-factor range that can be selected.

To decrease the effective indexes differences between polarizations or wavelengths, a waveguide design with a thicker thickness is necessary.

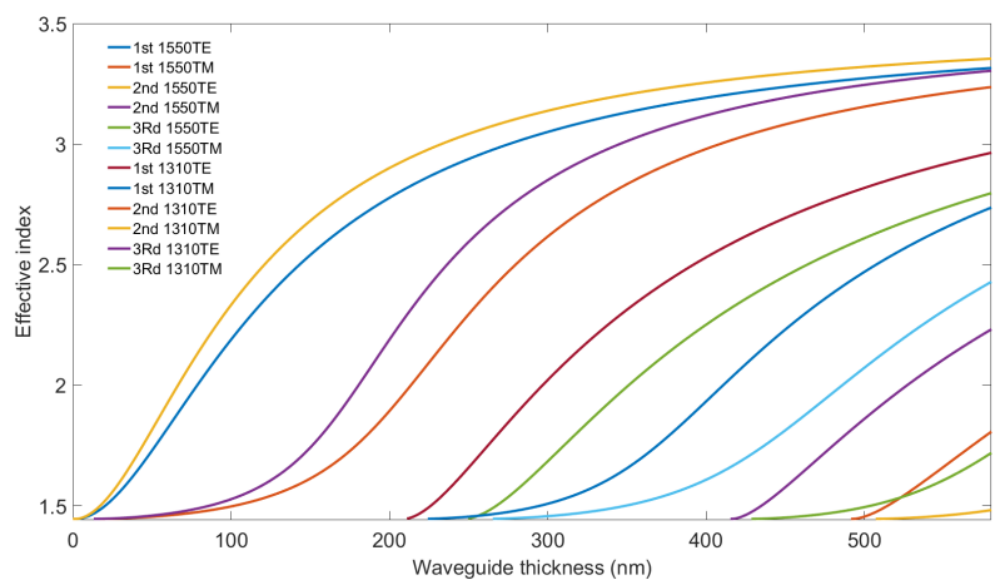

Figure 5.7 The effective index versus waveguide thickness for first, second, and third modes of 1550 $\mathrm{nm}$ and $1310 \mathrm{~nm}$.

As figure 5.7 shown, the effective indexes of different polarizations or wavelengths would get closer with a thicker waveguide. However, a thick waveguide will cause multimode diffractions in the waveguide, and the fundamental modes will be very lossy.

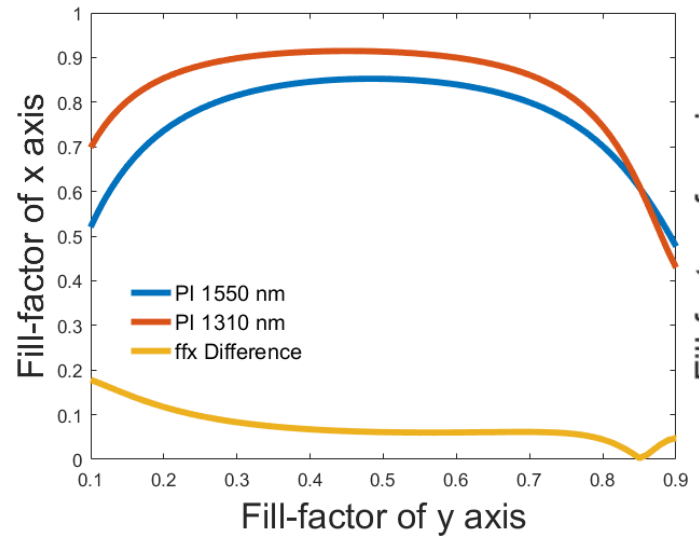

(a)

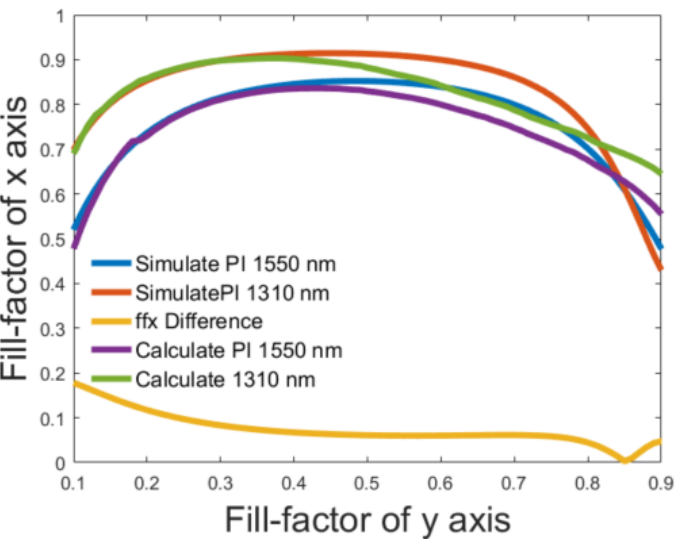

(b)

Figure 5.8 The polarization independent design of $\mathrm{ff}_{\mathrm{x}}$ versus $\mathrm{ff}_{\mathrm{y}}$ for (a) the calculation result and (b) comparison of simulation and calculation result. 
In figure 5.8, a $500 \mathrm{~nm}$ silicon waveguide would have a much smaller difference between the $1550 \mathrm{~nm}$ and $1310 \mathrm{~nm}$ for the polarization independent design. The difference between the polarization independent designs is smaller as figure 5.8 shown. The calculation result even have a cross point with $\mathrm{ff}_{y}$ around 0.85 . The simulation result is almost identical with the calculation result with a $\mathrm{ff}_{y}$ less than 0.5 , but it does not have a cross point with a large $\mathrm{ff}_{y}$ value. The difference between the simulation and calculation results is occurred because the EMT method is not accurate for very narrow square-shape holes. While the $\mathrm{ff}_{y}$ is very small or large, the grating structure would be more close to the zeroth-order EMT approximation. Although those two polarization independent design lines are getting closer with a thicker waveguide, it is still not enough to realize polarization independent for two bands at the same time.

To realize polarization independent for both $1310 \mathrm{~nm}$ and $1550 \mathrm{~nm}$, a new grating coupler concept based on the square-shape-hole structure with two additional shallow etched subwavelength slits, is proposed.

Fig 5.9 shows a schematic of the proposed polarization-independent wavelengthsplitting grating coupler with the two additional shallow etched subwavelength slit. It would use two-etching-step fabrication on an SOI platform with a height $h$ of $500 \mathrm{~nm}$. Similar to the grating couplers with square-shape holes shown in the previous section, this structure has fully etched square-shape-hole gratings with period lengths are named as $\Lambda_{x}$ and $\Lambda_{y}$ for $\mathrm{x}$ - and y-axis. $\mathrm{ff}_{x}$ and $\mathrm{ff}_{y}$ are the fill-factors of a grating period. This is the transverse subwavelength grating (SWG) region with a period length $\Lambda_{x}\left(1-\mathrm{ff}_{x}\right)$. The additional shallow-etched structure has a height of $h_{s u b}$ (where the etch depth is $h-h_{s u b}$ ) 
and have a total fill-factor $\mathrm{ff}_{\text {sub }}$ of the filling part of the $\mathrm{x}$-axis period as figure 5.9 (b) and (c) shown.

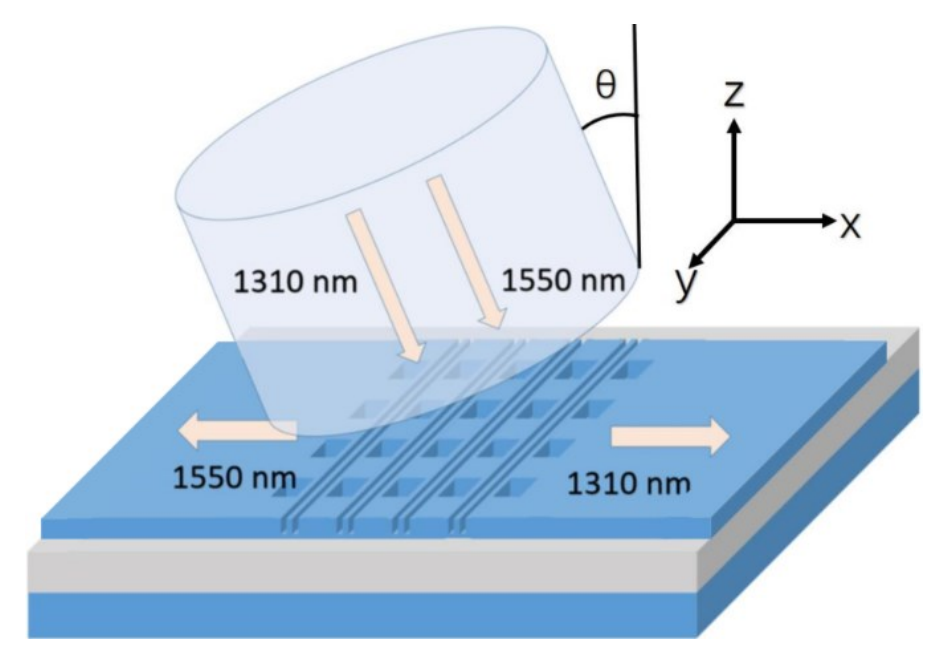

(a)

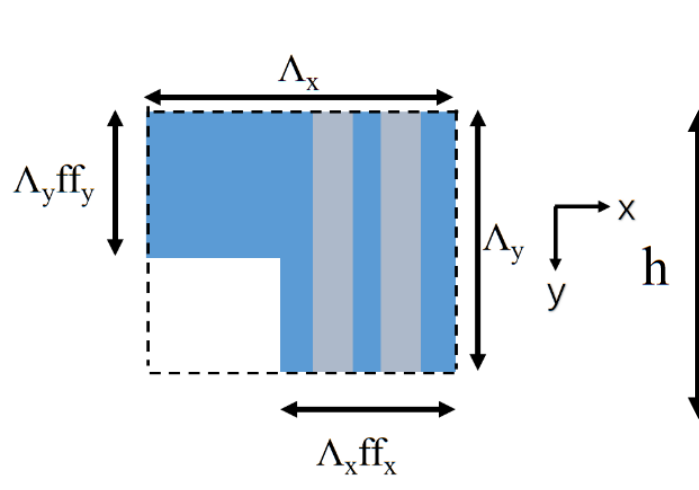

(b)

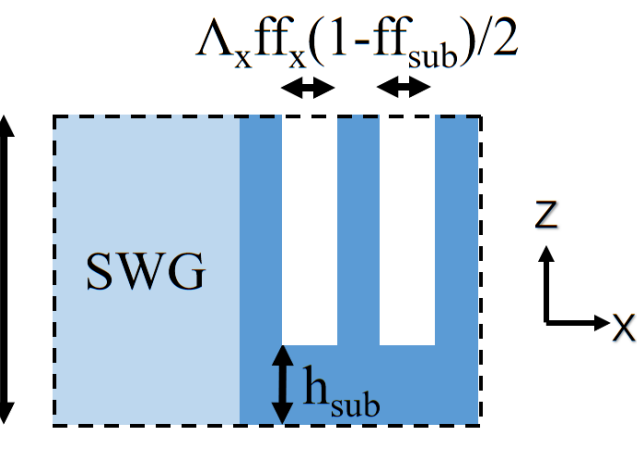

(c)

Figure 5.9 (a) Schematic representation of the polarization-independent wavelength-splitting

subwavelength grating coupler. (b) Top view of one period. (c) Side view of one period.

The design of the proposed dual-band grating coupler is accomplished in two steps.

First, polarization insensitivity is achieved by the transverse subwavelength region. As shown in [13], SWG structures can make the effective indices of the TE and TM fundamental Bloch-Floquet modes coincide at a given wavelength. Second, a series of shallow-etched slits are patterned in the homogeneous region to achieve $\theta(1310 \mathrm{~nm})=-\theta$ 
$(1550 \mathrm{~nm})$, while the polarization independence is maintained. This condition makes the coupled waves travel towards opposite directions, thereby enabling wavelength splitting.

The additional etched slits are etched more deeply (hse is less than $100 \mathrm{~nm}$ ). A deeply etched waveguide can guide light for a shorter wavelength better than a longer wavelength. Those subwavelength slits are necessary to decrease the difference between the two polarization independent lines. For the addition subwavelength pitches, they need to either decrease the effective index of $1550 \mathrm{~nm}$ TE mode more than $1310 \mathrm{~nm}$ light or increase the effective index of $1550 \mathrm{~nm}$ TM mode more than $1310 \mathrm{TE}$ mode. This can be realized because the changing rate of effective index difference between two polarizations of $1310 \mathrm{~nm}$ light and 1550 light are different.

With the initial value of $500 \mathrm{~nm}$ thickness and shallow etched fill-factor $\mathrm{ff}_{\text {sub }}=$ $0.5, \Lambda_{y}=400 \mathrm{~nm}$ and $h_{s u b}=80 \mathrm{~nm}$, the polarization independent design result after adding the subwavelength pitch is plotted in Figure 5.10. The polarization independent design has a y-direction fill-factor of 50\% and the $\mathrm{x}$-direction fill-factor of $60 \%$.

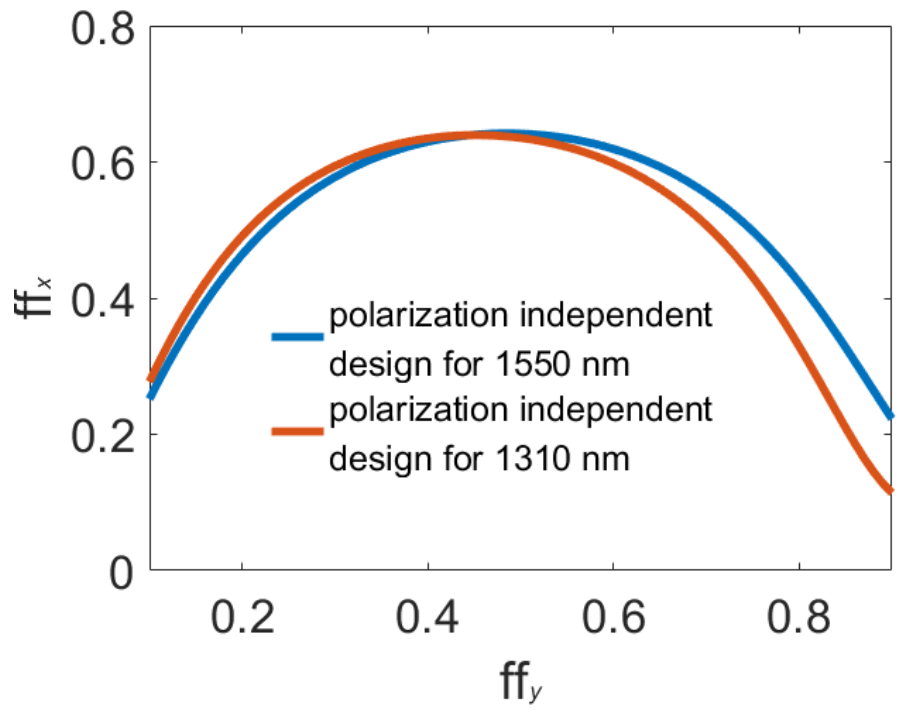

Figure 5.10 The $f f_{\mathrm{x}}$ versus $f f_{\mathrm{y}}$ that satisfy polarization independent for $1550 \mathrm{~nm}$ and $1310 \mathrm{~nm}$ in a grating coupler with subwavelength square-shape-hole structure. 
The fill-factor of the $y$-axis is in the more accurate range, as figure 5.8 (b) shown. In the meantime, a fill factor around 0.5 would have a transmission result larger than a very small or large fill-factor value. By using the fill-factors of the $\mathrm{x}$ - and $\mathrm{y}$-axis shown in figure 5.10 with Bragg condition, the incident angle versus the period length can be plotted.

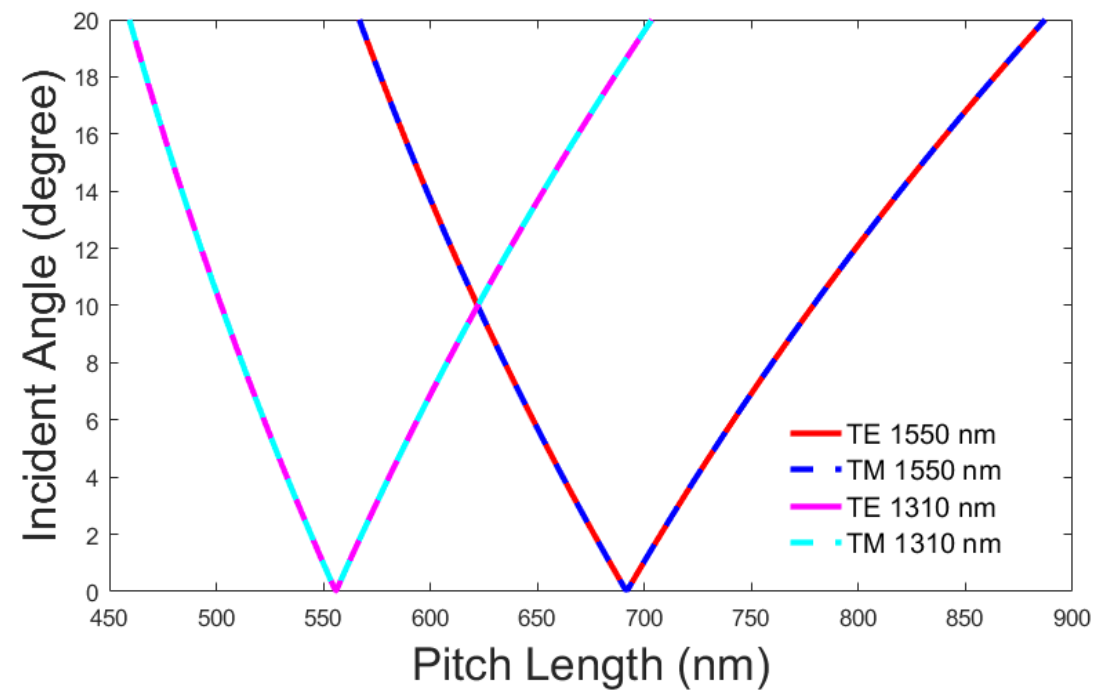

Figure 5.11 The incident angle versus period length that satisfies Bragg condition for the dual-band polarization independent grating coupler design

As figure 5.11 shown, the design lines for TE and TM polarizations of both 1550 $\mathrm{nm}$ and $1310 \mathrm{~nm}$ are crossing at the same point while the TE and TM polarizations' lines for single wavelength are identical. So that the parameters of a dual-band polarization independent grating coupler that can couple 1550nm light backward and $1310 \mathrm{~nm}$ light forwards can be observed from this figure.

Table 5.1 Design parameters for the dual-band polarization independent grating coupler

\begin{tabular}{|c|c|c|c|}
\hline $\boldsymbol{\Lambda}_{\mathbf{x}}$ & $660 \mathrm{~nm}$ & $\boldsymbol{\Lambda}_{\mathbf{y}}$ & $400 \mathrm{~nm}$ \\
\hline $\mathbf{f f}_{\mathbf{x}}$ & 0.6393 & $\mathbf{f f}_{\mathbf{y}}$ & 0.4455 \\
\hline $\mathbf{h}$ & $500 \mathrm{~nm}$ & $\boldsymbol{\theta ( 1 3 1 0} \mathbf{~ n m})$ & $9.99^{\circ}$ \\
\hline $\mathbf{h}_{\text {sub }}$ & $80 \mathrm{~nm}$ & $\mathbf{f f}_{\text {sub }}$ & 0.5 \\
\hline
\end{tabular}


The design parameters are listed in table 5.1. The FDTD simulation model can be built based on those data.

Because of the EMT approximation error of the square-shape-hole structure and the subwavelength slits, the simulation from the initial value has a low transmission and a large center wavelength mismatch. The optimized design result is shown in figure 5.12. Its design parameter is listed in table 5.2.

Table 5.2 Design parameters for the optimized dual-band polarization independent grating coupler

\begin{tabular}{|c|c|c|c|}
\hline$\Lambda_{\mathbf{x}}$ & $660 \mathrm{~nm}$ & $\boldsymbol{\Lambda}_{\mathbf{y}}$ & $410 \mathrm{~nm}$ \\
\hline $\mathbf{f f}_{\mathbf{x}}$ & 0.627 & $\mathbf{f f}_{\mathbf{y}}$ & 0.51 \\
\hline $\mathbf{h}$ & $500 \mathrm{~nm}$ & $\mathbf{\theta ( 1 3 1 0 ~ \mathbf { ~ m } )}$ & $9.5^{\circ}$ \\
\hline $\mathbf{h}_{\text {sub }}$ & $70 \mathrm{~nm}$ & $\mathbf{f f}_{\text {sub }}$ & 0.22 \\
\hline
\end{tabular}

From the 3D-FDTD simulation results shown in Fig. 5.12, the coupling efficiency for the TE and TM modes at $1310 \mathrm{~nm}$ is about $33 \%(-4.8 \mathrm{~dB})$ and $25 \%(-6 \mathrm{~dB})$, respectively, while the coupling efficiency for the TE and TM modes at $1550 \mathrm{~nm}$ is $27 \%(-5.7 \mathrm{~dB})$ and $15 \%(-8.2 \mathrm{~dB})$, respectively. Although the $1550 \mathrm{~nm}$ TM mode coupling efficiency is extremely low while the back reflection at $1310 \mathrm{~nm}$ TE is very strong, these results still pave the way for the implementation of a fully operative polarization-independent wavelength-demultiplexing grating coupler. 


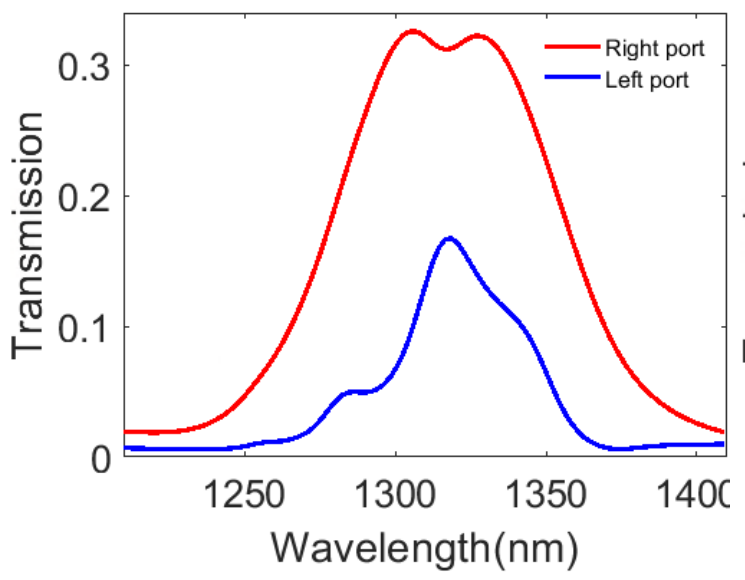

(a)

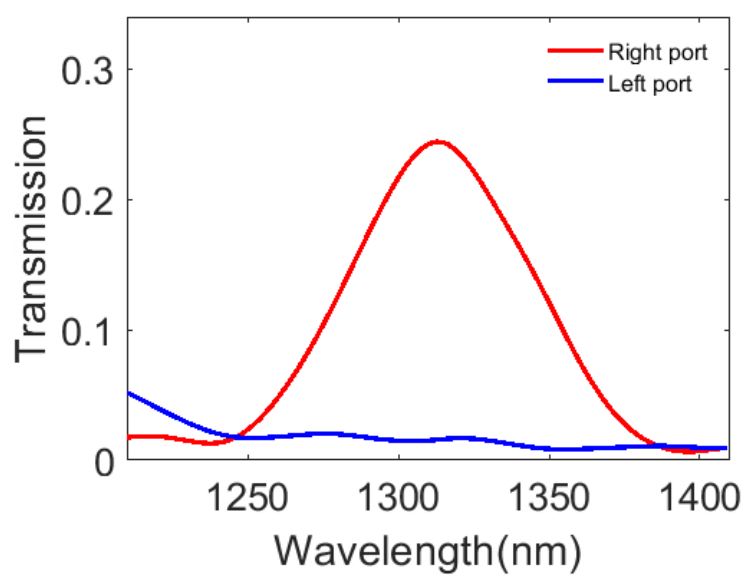

(c)

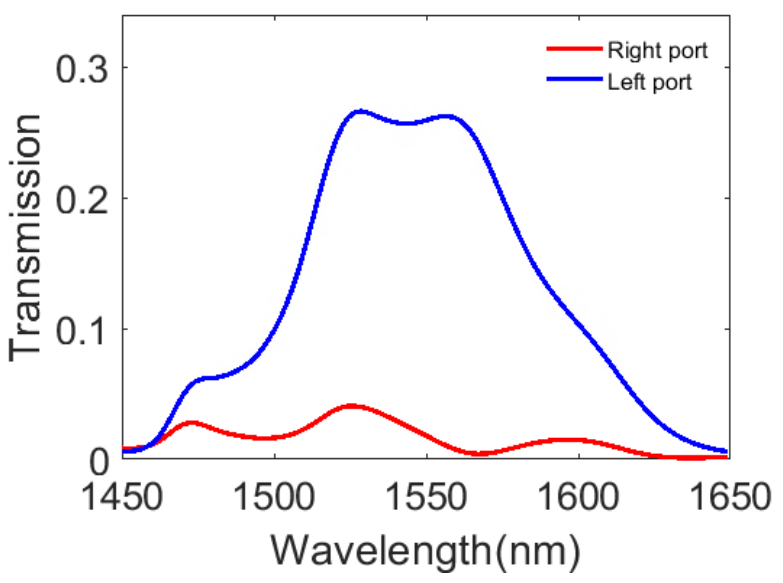

(b)

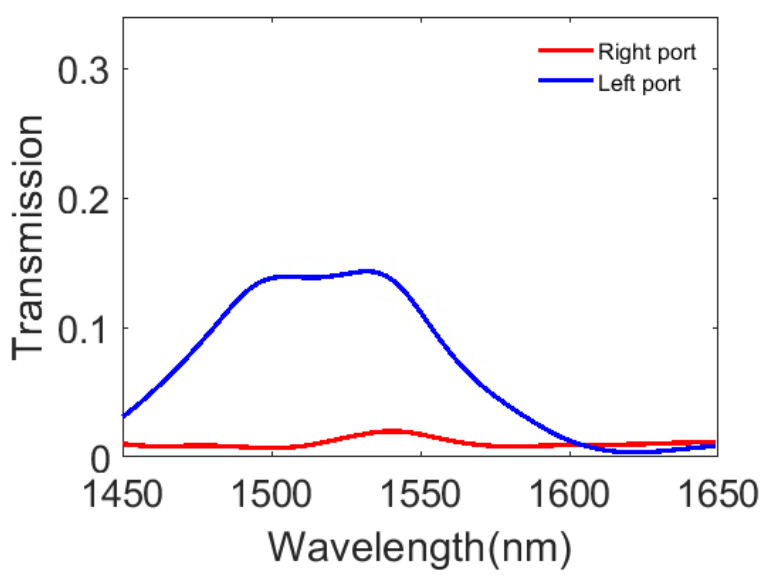

(d)

Figure 5.12 Coupling efficiency as a function of the wavelength for (a) O-band [TE], (b) C-band [TE], (c) O-band [TM] and (d) C-band [TM].

\subsection{Optimization and Tolerance Analysis of the Dual-Band Polarization} Independent Grating Coupler.

The dual-band polarization independent design shown in section 5.2 has the disadvantage that the minimum feature size is around $30 \mathrm{~nm}$ (the width of the subwavelength slits). The $30-\mathrm{nm}$ minimum feature size is almost impossible to be fabricated. A novel fabricable optimized structure is shown below. 


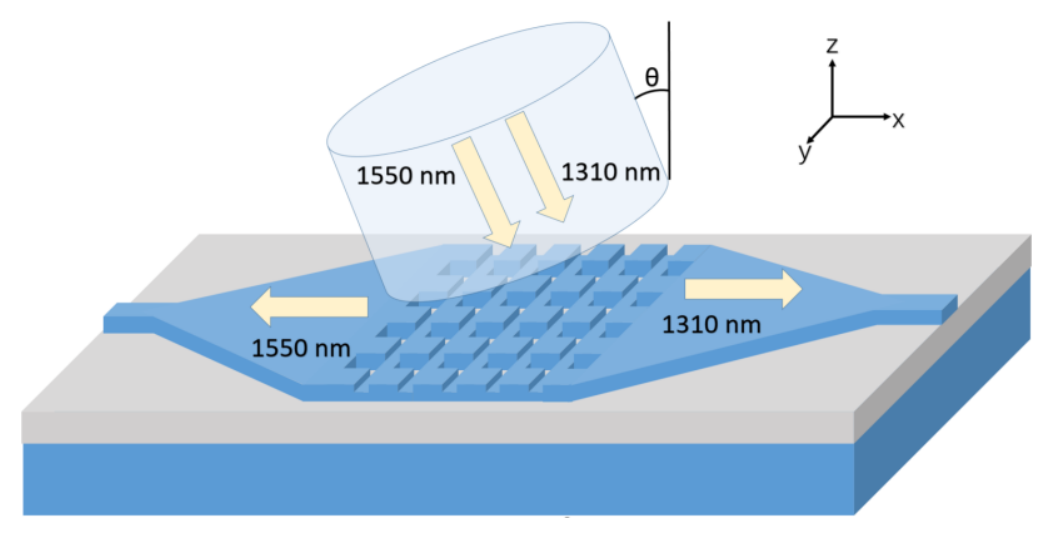

(a)

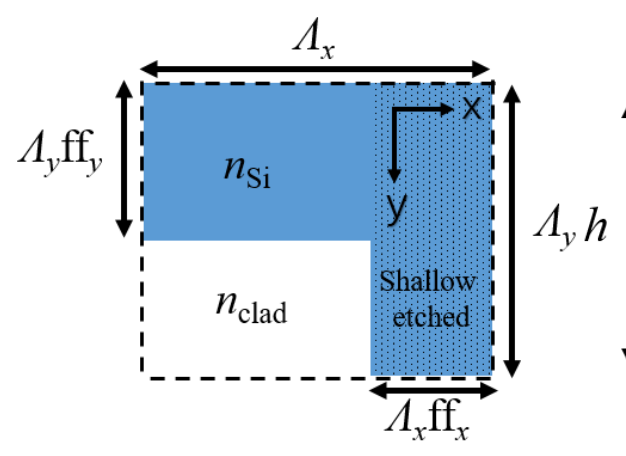

(b)

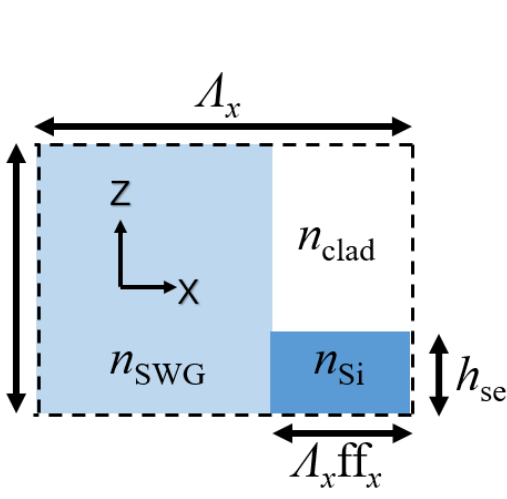

(c)

Figure 5.13 (a) Schematic representation of the polarization-independent wavelength-splitting subwavelength grating coupler. (b) Top view of one period. (c) Side view of one period.

In this structure shown in figure 5.13, a shallow etched fill part of $\mathrm{x}$-axis $\left(\Lambda_{x} \mathrm{ff} x_{x}\right.$ part) is used to replace the subwavelength slits in figure 5.9. Both the shallow etched structure and subwavelength slits are used for decreasing the difference between the polarization independent design lines (as figure 5.8 shown). Because the shallow etched structure can change the effective index of the structure more than the subwavelength slits did, the waveguide has a thickness of $360 \mathrm{~nm}$. With an etch depth of $280 \mathrm{~nm}$, the polarization independent plot can be shown as the following: 


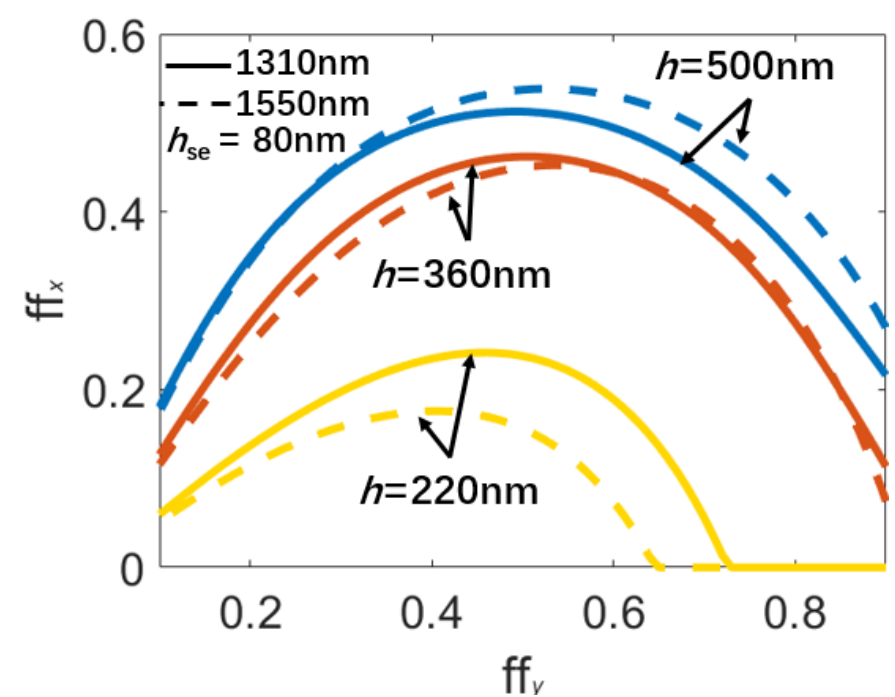

Figure 5.14 The $\mathrm{ff}_{\mathrm{x}}$ versus $\mathrm{ff}_{\mathrm{y}}$ that satisfy polarization independent for $1550 \mathrm{~nm}$ and $1310 \mathrm{~nm}$ in the dual-band polarization independent grating coupler design

By using this new structure, the polarization independent design for $1310 \mathrm{~nm}$ and $1550 \mathrm{~nm}$ are almost identical with a fill-factor of $y$-axis at 0.63 . The optimized design parameters are listed in table 5.3, and its simulation results are shown in figure 5.15. Figure 5.15 also present the calculated results with thickness $220 \mathrm{~nm}$ and $500 \mathrm{~nm}$. It is clearly that $220 \mathrm{~nm}$ thickness cannot realize dual-band polarization insensitivity and $500 \mathrm{~nm}$ thickness might have a strong multimode effect. This 360-nm-thick structure has a minimum feature size of $150 \mathrm{~nm}$. This dimension of the gratings is fabricable.

Table 5.2 The data of the polarization independent wavelength splitting grating coupler

\begin{tabular}{|c|c|c|c|}
\hline Axis & Pitch length & Pitch number & Duty cycle \\
\hline $\mathrm{x}$ & $735 \mathrm{~nm}$ & 13 & 0.385 \\
\hline $\mathrm{y}$ & $300 \mathrm{~nm}$ & 30 & 0.56 \\
\hline Silicon height & Etched depth & Incident angle & SiO2 Thickness \\
\hline $360 \mathrm{~nm}$ & $280 \mathrm{~nm}$ & 9 degrees & $2 \mu \mathrm{m}$ \\
\hline
\end{tabular}




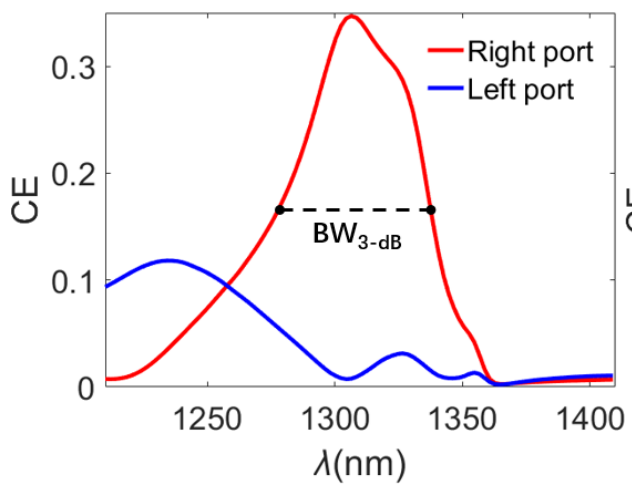

(a)

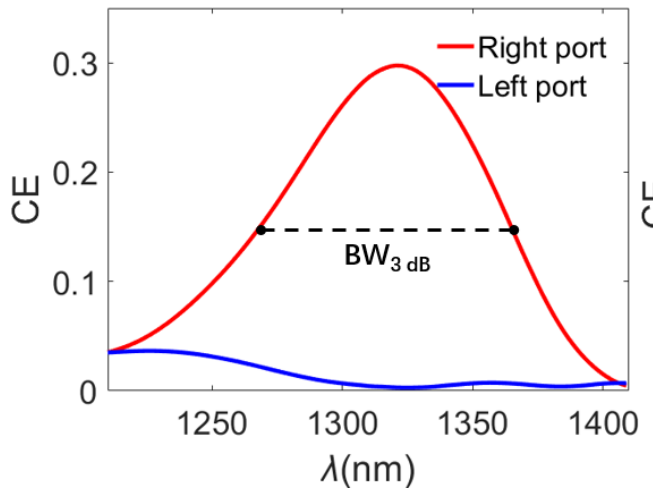

(c)

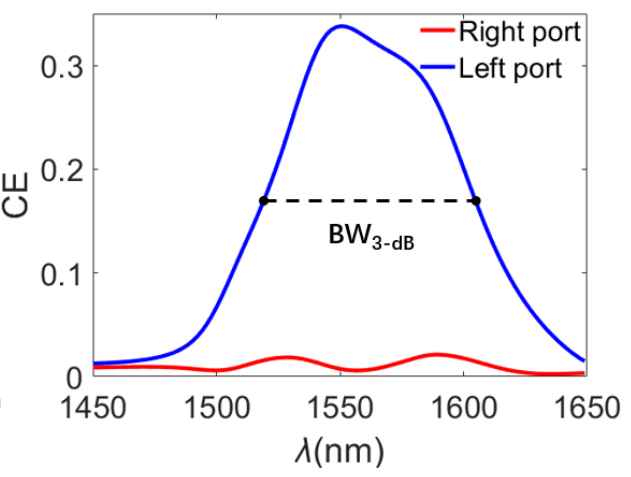

(b)

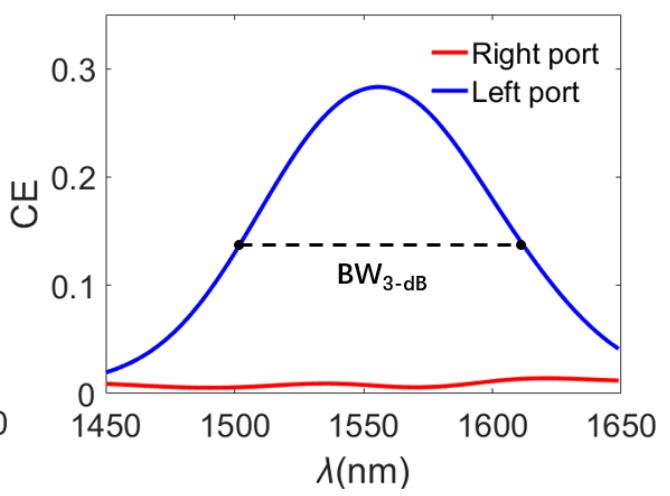

(d)

Figure 5.15 Coupling efficiency of the dual-band polarization independent grating coupler for (a) $\mathrm{O}$ band [TE], (b) C-band [TE], (c) O-band [TM] and (d) C-band [TM].

From the 3D-FDTD simulation result shown in figure 5.15, the coupling efficiency for the TE and TM modes at both O-band and C-band is about 34\% and 29\%, respectively. The back reflections of the designed grating coupler at the center wavelength are $1 \%-2.5 \%$. The weak back reflection is at least $16 \mathrm{~dB}$ less than the coupling mode. For the wavelength less than $1280 \mathrm{~nm}$, the back reflection of the TE mode is influential.

The optimization for two polarizations of two wavelengths is challenging because the coupling spectrum of this grating coupler is dependent on $\mathrm{ff}_{\mathrm{x}}, \mathrm{ff}_{\mathrm{y}}, \Lambda_{\mathrm{x}}, \Lambda_{\mathrm{y}}, \theta$, and $e d$. The original y-direction pitch length $(390 \mathrm{~nm})$ using was not suited because the back reflection 
is strong right at $1310 \mathrm{~nm}$ for the TE mode. After optimization, a $300 \mathrm{~nm}$ y-direction period length has been chosen.

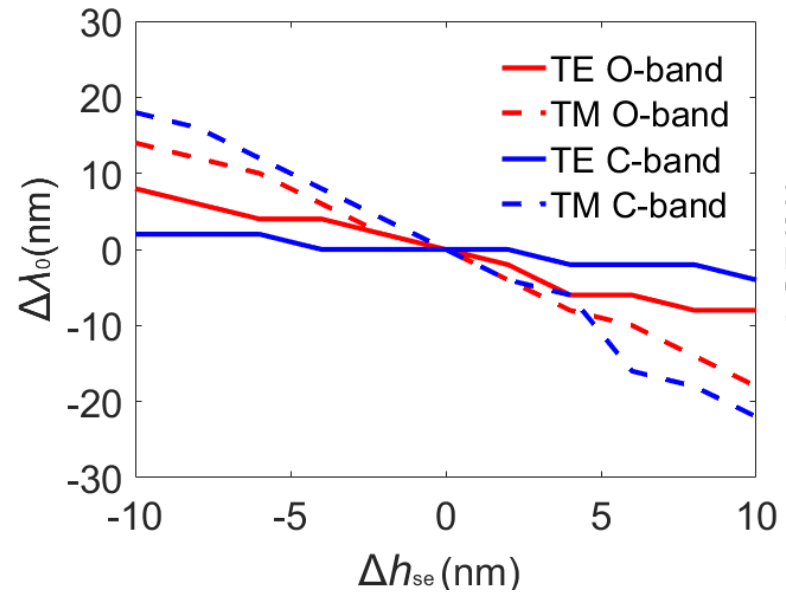

(a)

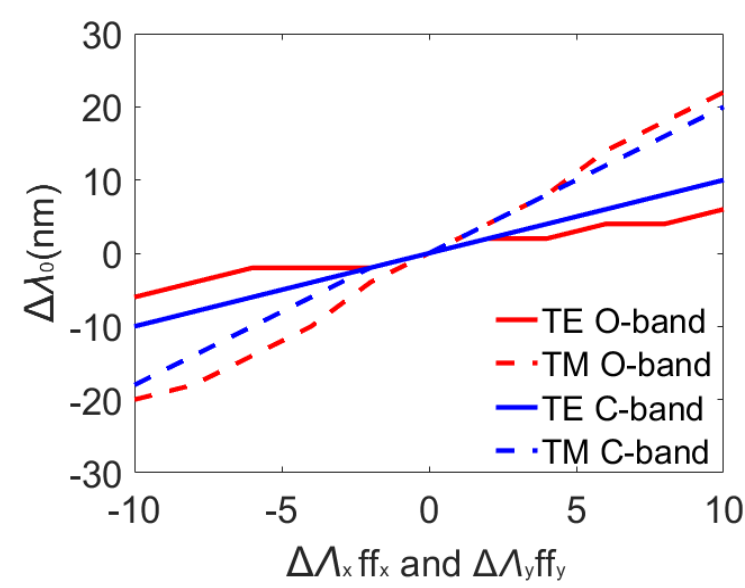

(c)

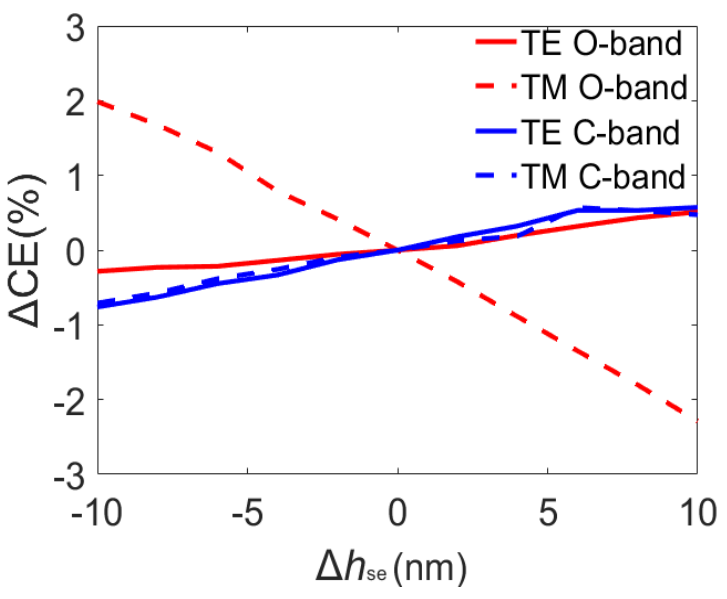

(b)

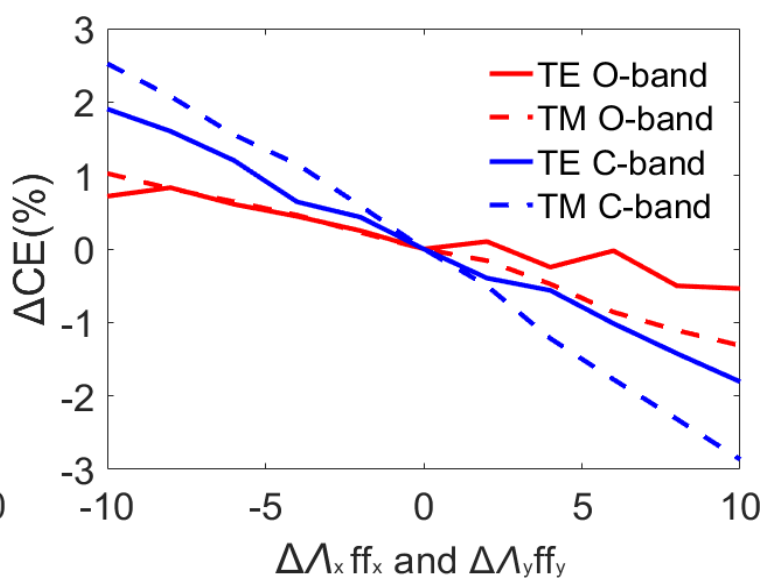

(d)

Figure 5.16 Tolerance analysis of the designed dual-band polarization-insensitive wavelengthsplitting grating coupler. (a) Central wavelength and (b) coupling efficiency deviation as a function of errors in the shallow-etched depth. (c) Central wavelength and (d) coupling efficiency deviation as a function of errors in the length $\left(\Delta \Lambda_{x} f f_{x}\right)$ and width $\left(\Lambda_{y} f f_{y}\right)$ of the grating teeth.

Two steps are required to fabricate the proposed grating coupler: to define fully etched SWG sections and to shallow-etch the silicon sections. In Figure 5.16, we study the influence of fabrication deviations in the etch depth $\left(\Delta h_{\text {se }}\right)$ and the size of grating teeth 
$\left(\Delta \Lambda_{\mathrm{x}} \mathrm{ff}_{\mathrm{x}}\right.$ and $\Delta \Lambda_{\mathrm{y}} \mathrm{ff}_{\mathrm{y}}$ simultaneously) with respect to the nominal values, using 3D FDTD. Fabrication errors lead to a maximum wavelength shift $\Delta \lambda 0$ of $\pm 20 \mathrm{~nm}$ with respect to the central operating wavelengths of the grating couplers (Figure 5.16 (a) and (c)), while the degradation of the coupling efficiency $(\triangle \mathrm{CE})$ is less than $3 \%$ in all cases. 


\section{Chapter 6:}

\section{Conclusion and Future Work}

This thesis presented the theory and design flow of dual-wavelength grating couplers. The thesis involves three different designs for polarization and wavelength manipulation:

1. A 1-D vertical incident dual-wavelength grating coupler for (de)multiplexing. The optimized design achieved a maximum coupling efficiency of around $20 \%$ with $\sim-10 \mathrm{~dB}$ low crosstalk. The $3-\mathrm{dB}$ bandwidths of $1310 \mathrm{~nm}$ and $1550 \mathrm{~nm}$ is $120 \mathrm{~nm}$ and $150 \mathrm{~nm}$, respectively. (Chapter 3)

2. A 2-D four-output-port grating coupler for polarization and wavelength splitting with flexible incident angles, for $1310 \mathrm{~nm}$ and $1550 \mathrm{~nm}$. This design has transmissions of $\sim 30 \%$ for $1310 \mathrm{~nm}$ TE and $1550 \mathrm{~nm} \mathrm{TM}$, and $\sim 12 \%$ for $1310 \mathrm{~nm}$ TM and $1550 \mathrm{~nm}$ TE. The design shows the polarization crosstalk of maximum $-9 \mathrm{~dB}$. (Chapter 4)

3. A dual-band (O- and $\mathrm{C}-$ band) polarization independent 3-D grating couplers for wavelength (de)multiplexing on a 360-nm-thick or 500-nm-thick SOI platform. The final optimized design has a coupling efficiency of $\sim 30 \%$ and a $3-\mathrm{dB}$ bandwidth of $\sim 90 \mathrm{~nm}$ for both polarizations of $1310 \mathrm{~nm}$ and $1550 \mathrm{~nm}$. The wavelength crosstalk is less than $-16 \mathrm{~dB}$ when this design works as a fiber-tochip grating coupler. (Chapter 5)

The key design procedure in grating couplers is the effective index engineering of the grating structures. By adjusting the fill-factor and the etched depth of the gratings, the 
average effective index of the selected propagating mode can be controlled. The average effective index, the incident angle, and the period length of the structure would follow the Bragg condition and contribute to the performance at the chosen operating wavelength(s). In particular, the 1-D grating couplers have their limitations in fabrication and design flexibility. Typical deep-UV fabrication processes require a minimum feature size of 75$100 \mathrm{~nm}$ [52]. In fabrication, the etching process is generally easier and faster to be realized than the material growth process. Due to the strong wavelength and polarization sensitivity of the grating couplers, L-shape, U-shape, and inverted-T-shape gratings were introduced to provide additional design freedom to grating designs and to improve the performance characteristics. Compared to the 1-D grating designs, the 2-D grating couplers have one more dimension to offer in the engineering of the structural design. With techniques such as subwavelength segmentation or apodization of the structure, grating couplers are able to achieve higher coupling efficiencies.

The vertical incident dual-wavelength grating coupler has low coupling efficiencies because most of the power is lost in the substrate of the grating. In fact, for this same reason, most vertical incident grating coupler has low transmissions. By adding a bottom reflector such as a distributed Bragg reflector or an Au reflector, the coupling efficiency can be at least doubled, if not more. However, it may not be feasible to add a bottom reflector. Au is not CMOS compatible, making it an invalid option for any of the CMOS fabrication processes. As for Bragg stacks, the wavelength sensitivity would be a major limiting factor if broadband is a design specification.

In this thesis, designing a dual-band polarization independent grating coupler is achieved in chapter 5 . The final optimized grating coupler has a simple structure and 
achieves a higher coupling efficiency than the grating structures demonstrated previously in literature. More importantly, the polarization insensitivity makes this grating design more suitable to support communication systems with increased data rates.

\subsection{Significance of Thesis Work}

Based on the research work conducted in this thesis, 2 publications were submitted:

1. T. Hao, A. Sánchez-Postigo, P. Cheben, A. Ortega-Moñux, and W. N. Ye, "DualBand Polarization-Independent Subwavelength Grating Coupler for Wavelength Demultiplexing", IEEE Photonics Technology Letters (submitted, June $02^{\text {nd }}, 2020$ ).

2. T. Hao, A. Sánchez-Postigo, P. Cheben, and W. N. Ye, "Dual-Band PolarizationIndependent Subwavelength Grating Coupler for Wavelength Demultiplexing”, 2020 IEEE Group IV Photonics / 2020 IEEE Photonics Conference, Vancouver, Sept 28 $8^{\text {th }}, 2020$. (submitted in May, 2020).

The conference paper is based on the dual-band polarization independent design with subwavelength slits and the PTL paper is based on the dual-band polarization independent design with shallow-etched and SWG gratings.

\subsection{Future Work}

One variation of our grating coupler design is a wavelength and polarization splitting grating coupler using a constant incident angle, as shown in Figure 6.1. In chapter 4, a similar structure was proposed and designed, but poor performance in coupling efficiency and strong crosstalk were demonstrated. Instead of using a constant incident angle, a flexible radiation angle should be adopted. Additional analysis on different core thicknesses or varied subwavelength structure may be helpful to realize a more efficient 
polarization and wavelength splitting design. Alternatively, one ultimate design is to demonstrate wavelength and polarization-insensitive grating coupler with a single output port.

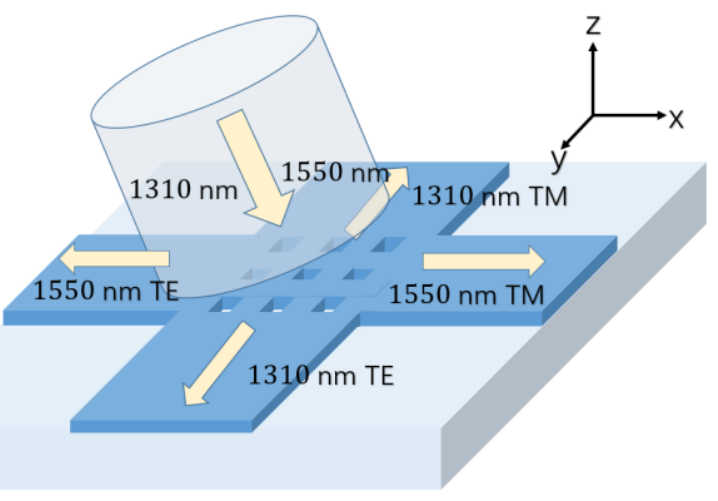

Figure 6.1 Schematic of a polarization and wavelength splitting grating coupler

The experimental demonstration of the novel dual-wavelength polarization independent grating coupler design presented in Chapter 5 could also be done in the future for verification. Although this design has not been fabricated, it has a minimum feature size of $\sim 150 \mathrm{~nm}$. This feature size is feasible by fabrication using the deep-UV fabrication processes.

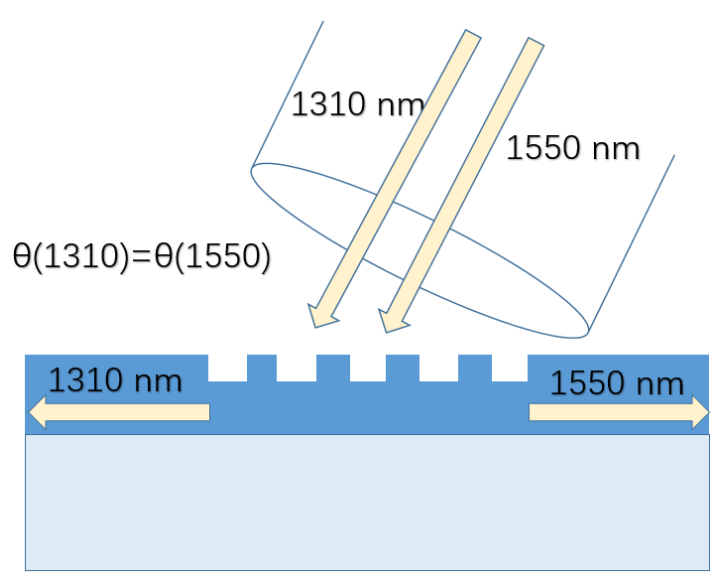

(a)

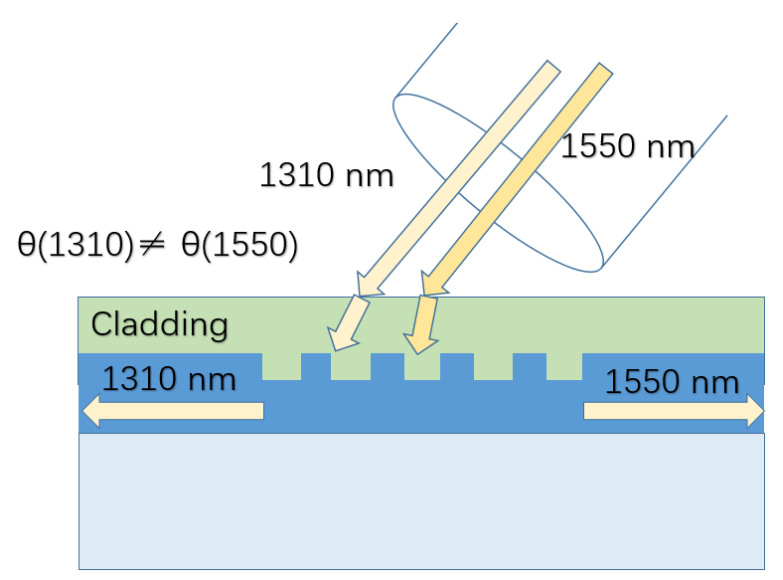

(b)

Figure 6.2 Schematics of a dual-wavelength grating coupler (a) without cladding and (b) with a specially designed cladding 
Further improvements can be done for the dual-wavelength grating coupler design. For example, bottom reflectors can be used to increase the coupling efficiency. A specially designed cladding layer can provide additional control in the incident angle, to couple light into the grating coupler. As illustrated in Figure 6.2 (b), the cladding guides light beams at $1310 \mathrm{~nm}$ and $1550 \mathrm{~nm}$ into the grating coupling at different angles due to the dispersion properties of the cladding material. A cladding with a strong dispersion property can induce larger refraction angle for different wavelengths. However, it will be challenging to find the appropriate cladding material(s) to offer us the specific angels at desired wavelengths. 


\section{Appendices}

\section{Appendix A FDTD Scripts to generate grating coupler}

\section{A.1 Code to generate a fiber}

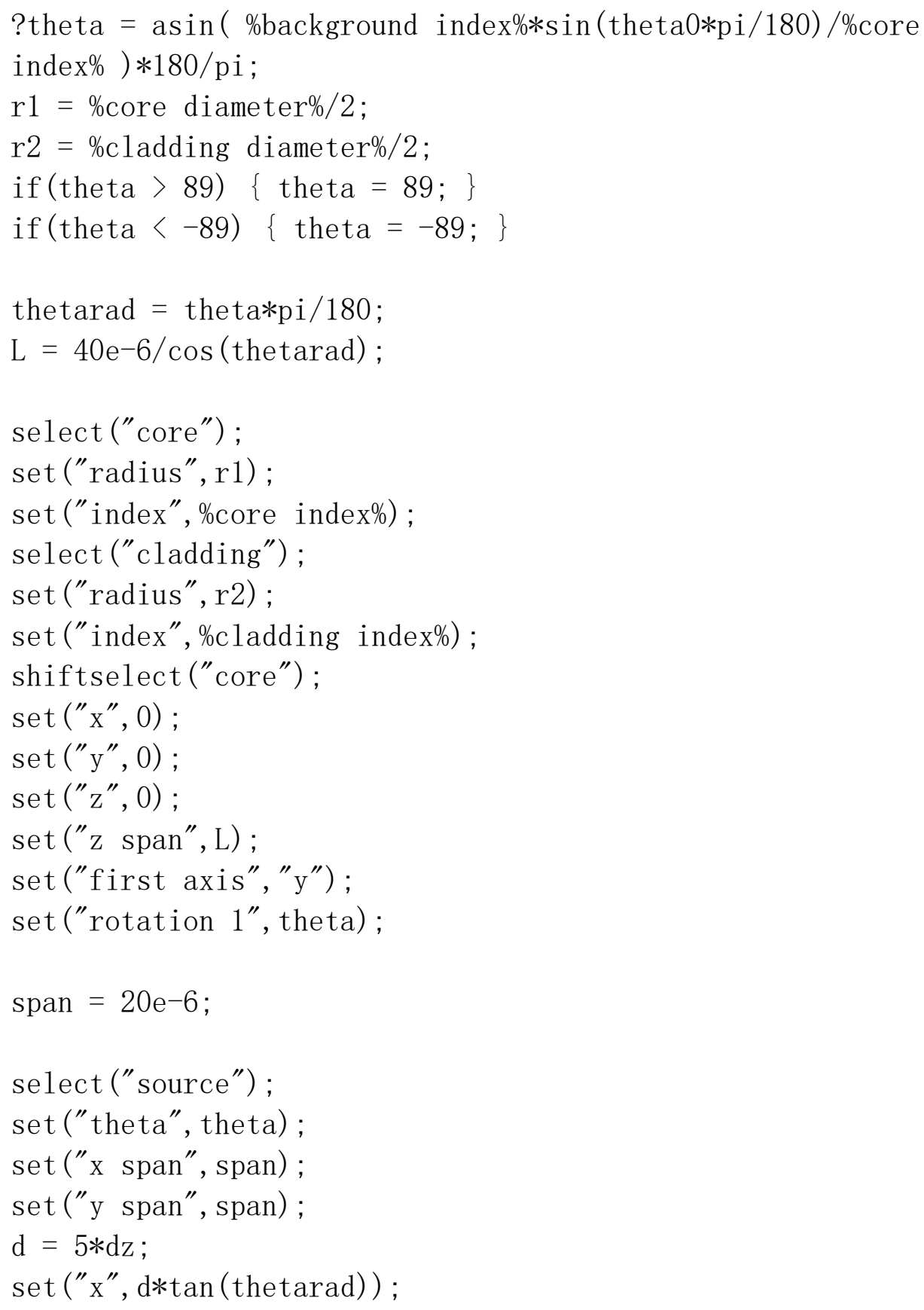




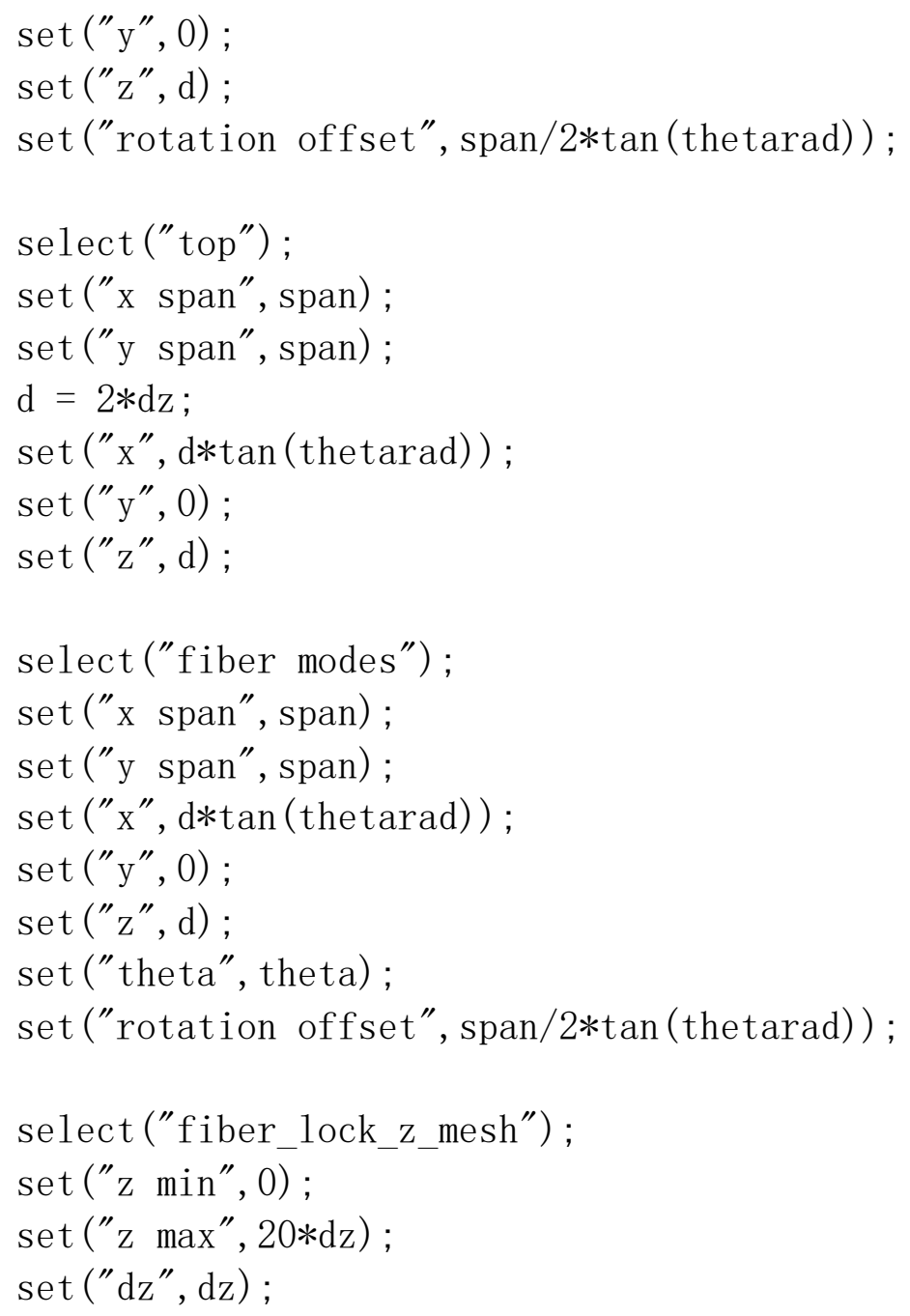

\section{A.2 Code to generate a vertical incident dual-band grating coupler}

deleteal1;

n_periods = ceil (\%target length\%/2/pitch);

n_periods2 = cei1 (\%target length\%/2/pitch2);

fil1_width $=$ pitch $* \%$ duty cycle $\%$;

etch_width $=$ pitch $*(1-\%$ duty cycle\%);

fil1_width2 $=$ pitch $2 * \%$ duty cycle\%;

etch_width2 $=$ pitch $2 *(1-\%$ duty cycle\% $)$;

L1 = (n_periods -1$) *$ pitch + etch_width;

$\mathrm{L} 2=$ n_periods $2 *$ pitch2 + etch_width2;

$\mathrm{xxx}=\mathrm{pitch} *\left(\mathrm{n} \_\right.$periods -1$)+$ etch_width ; 


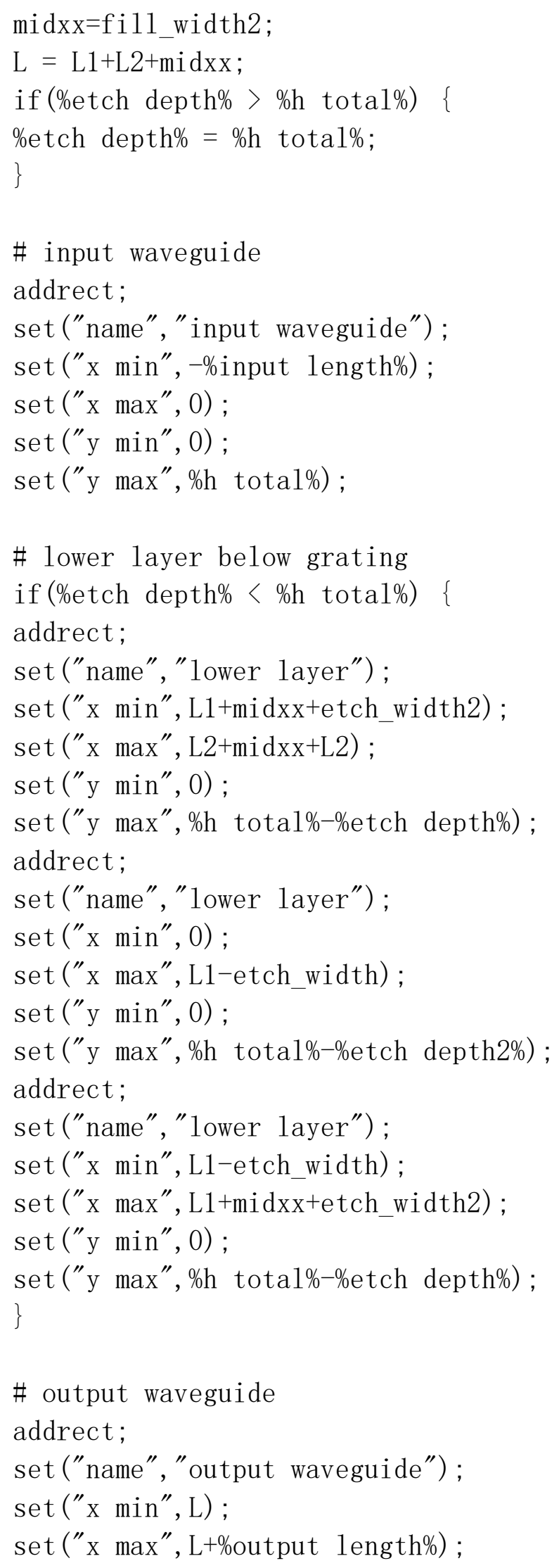




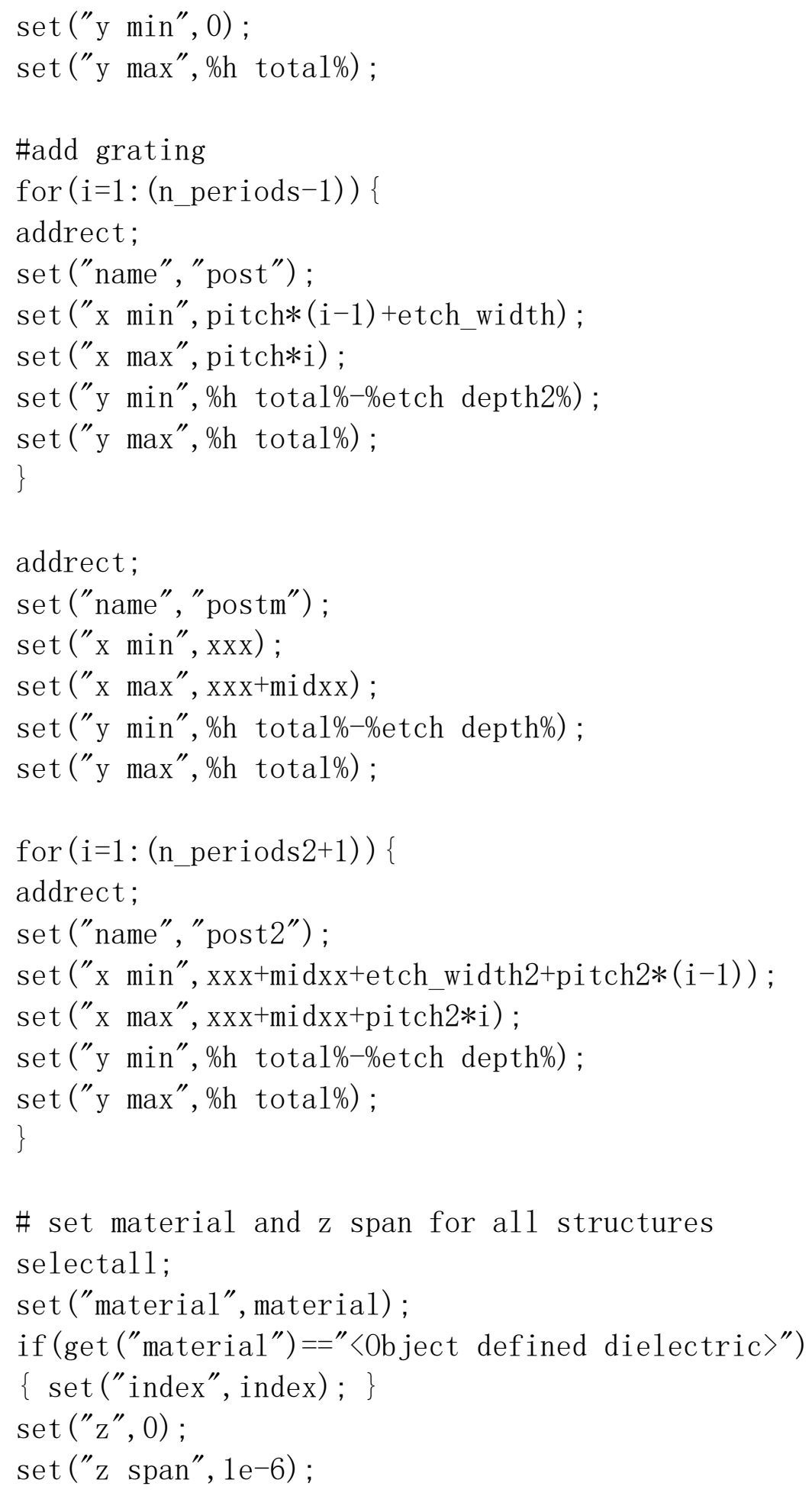




\section{A.3 Code to generate a dual-band polarization independent subwavelength grating coupler for wavelength demultiplexing}

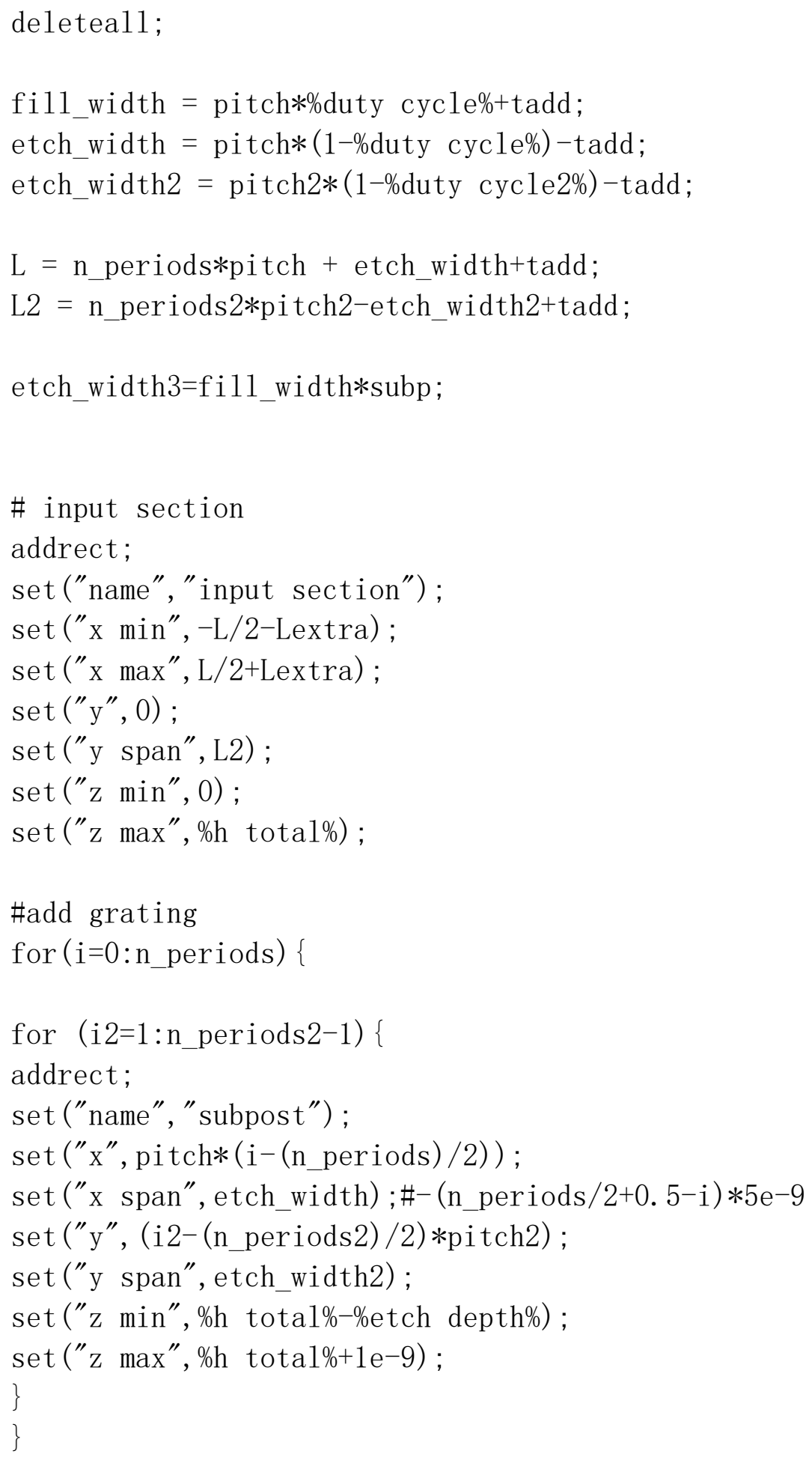




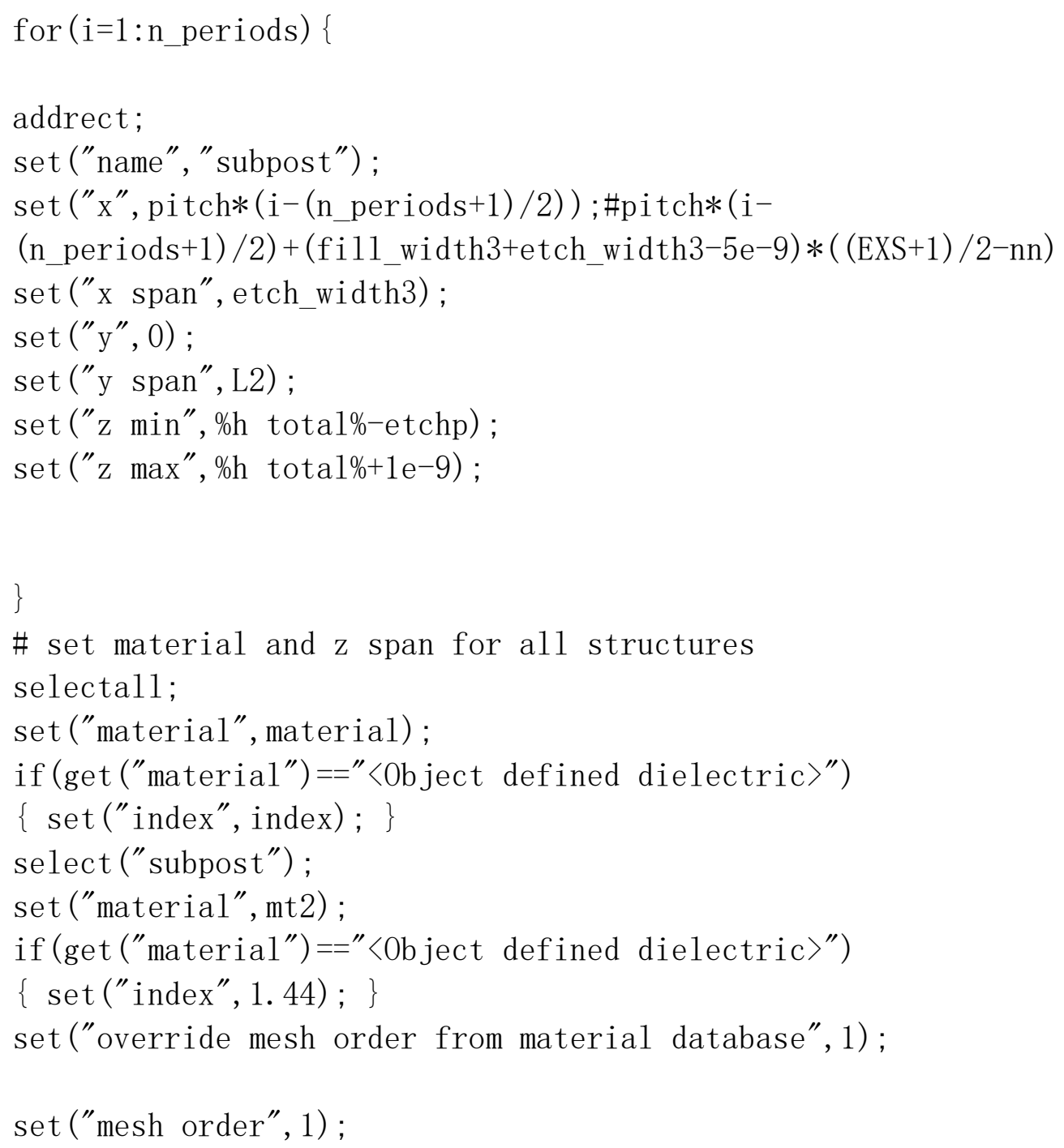




\section{Appendix B Analysis Scripts}

\section{B.1 Matlab Code for 1-D Effective Index Approximation}

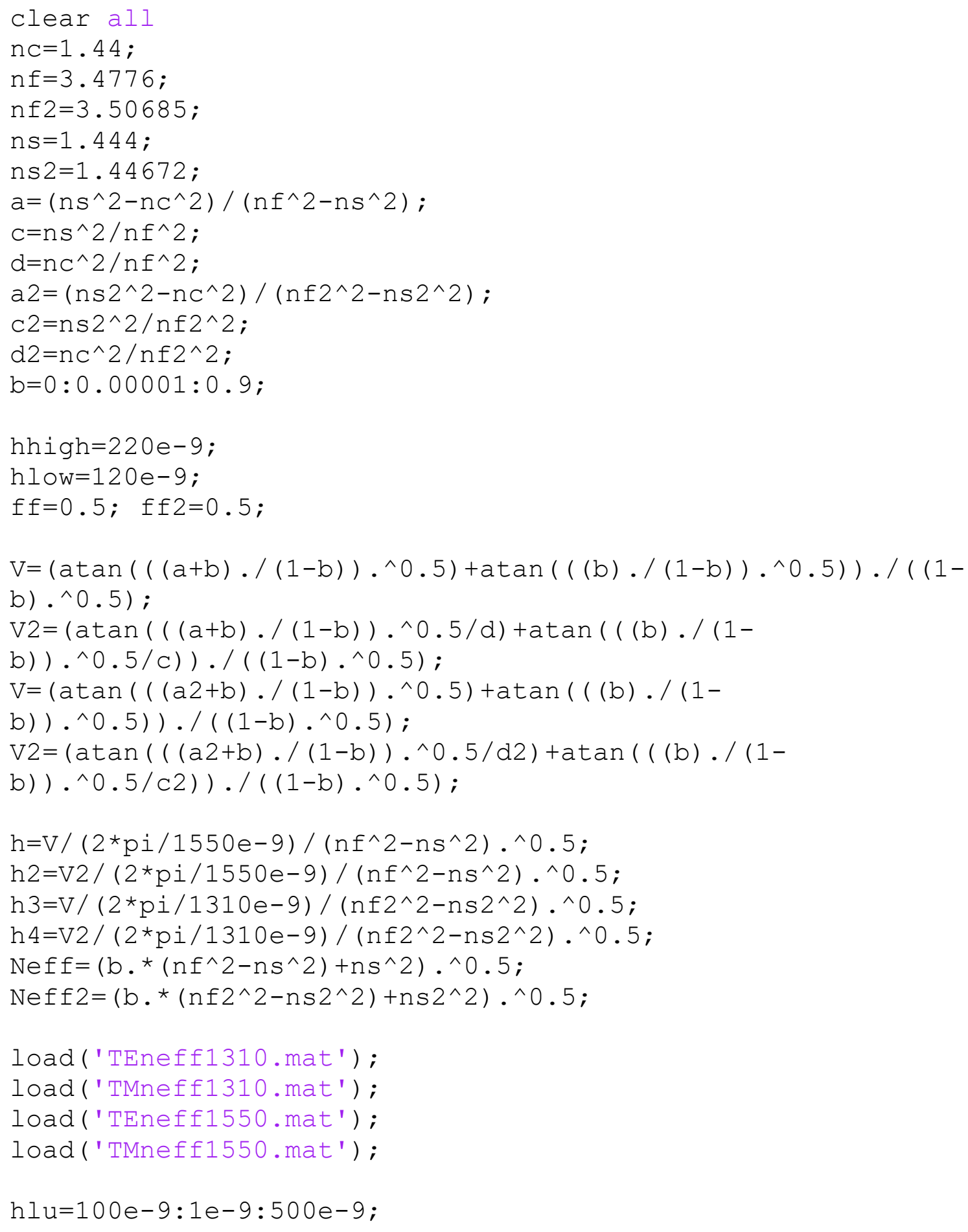


plot (h, Neff,h2, Neff,h3, Neff,h4, Neff);

hold on;

plot (hlu, TEneff1550, hlu, TMneff1550,hlu, TEneff1310, hlu, TMnef f1310)

$x \lim ([100 e-9500 e-9])$;

xlabel ('Waveguide hight (nm)')

ylabel ('Reflective index')

legend('1550nm TE',' 1550 nm TM',' '1310nm TE','1310 nm TM');

legend ( 'boxoff')

\section{B.2 Plot the incident angle versus period length from the Bragg condition}

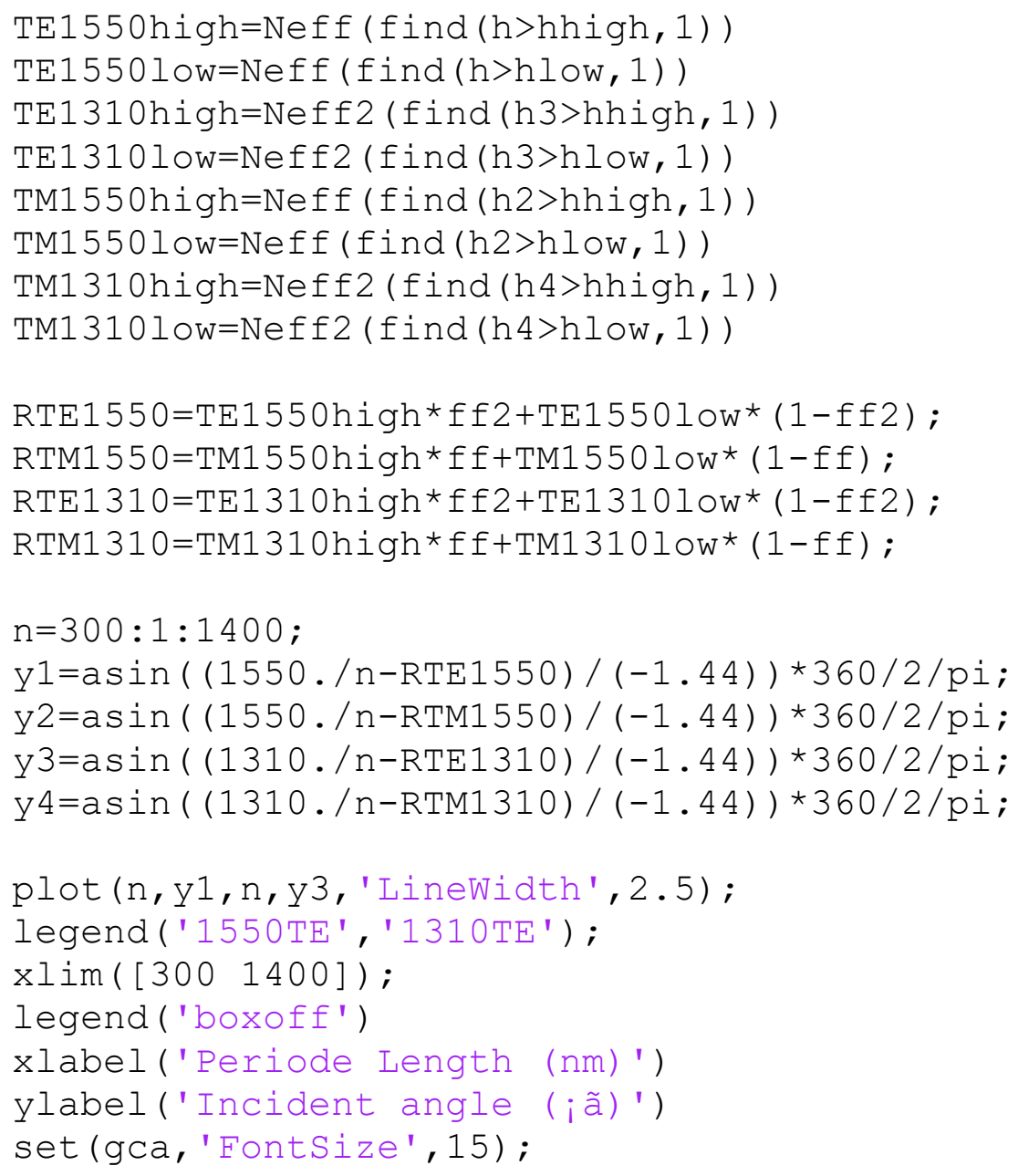

\section{B.3 Parameter sweep for 2-D grating coupler}

left1 $=\operatorname{matrix}(6,100)$;

right $1=\operatorname{matrix}(6,100)$; 


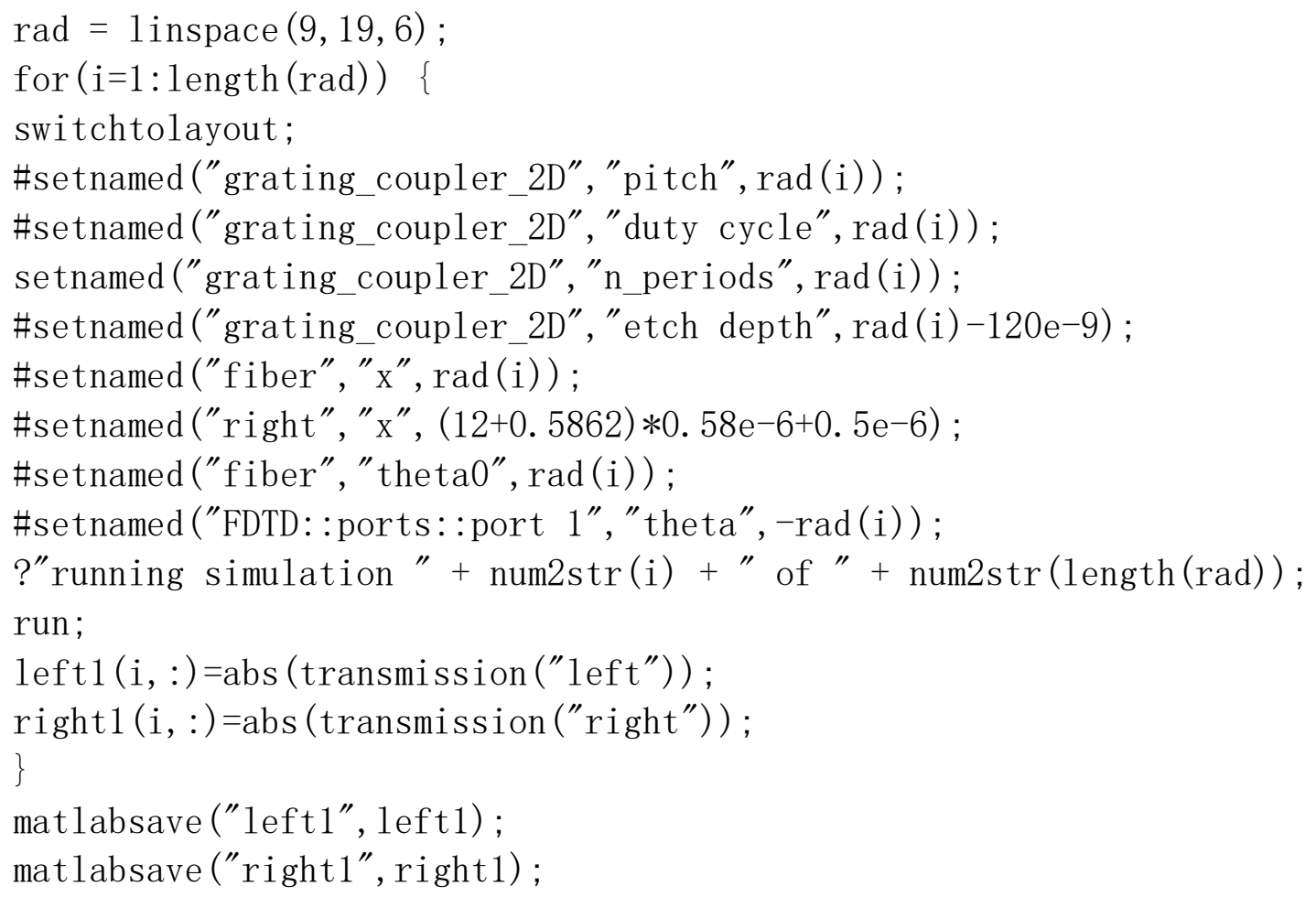

\section{B.4 Matlab code for Polarization Independent Calcualtion}

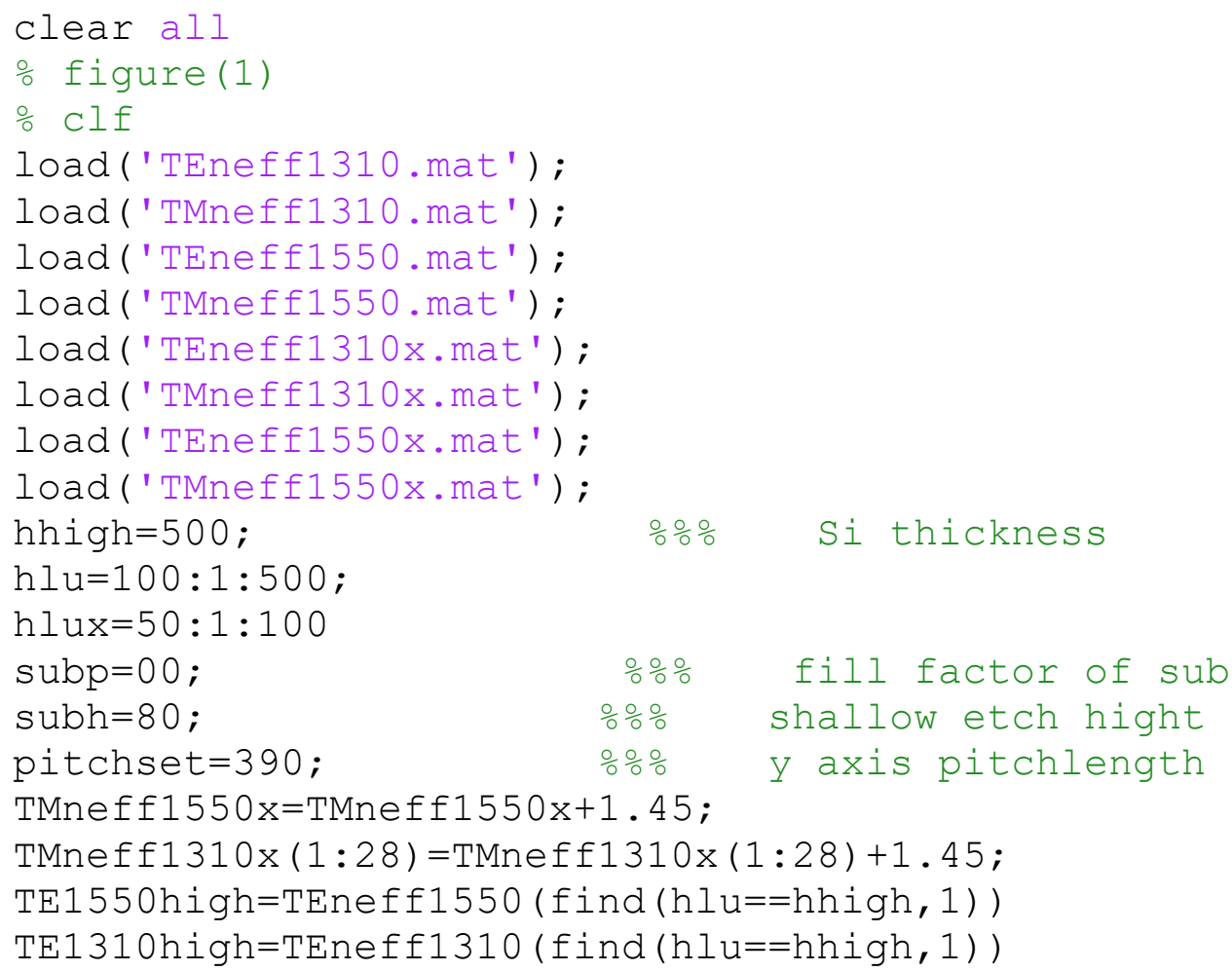




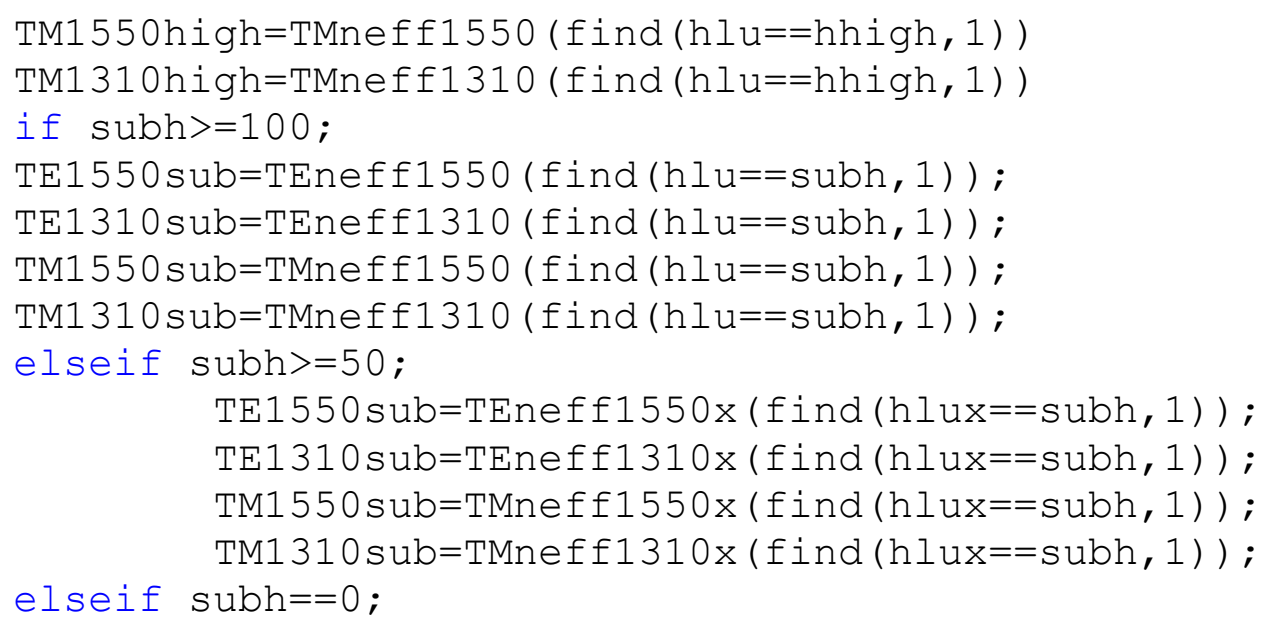




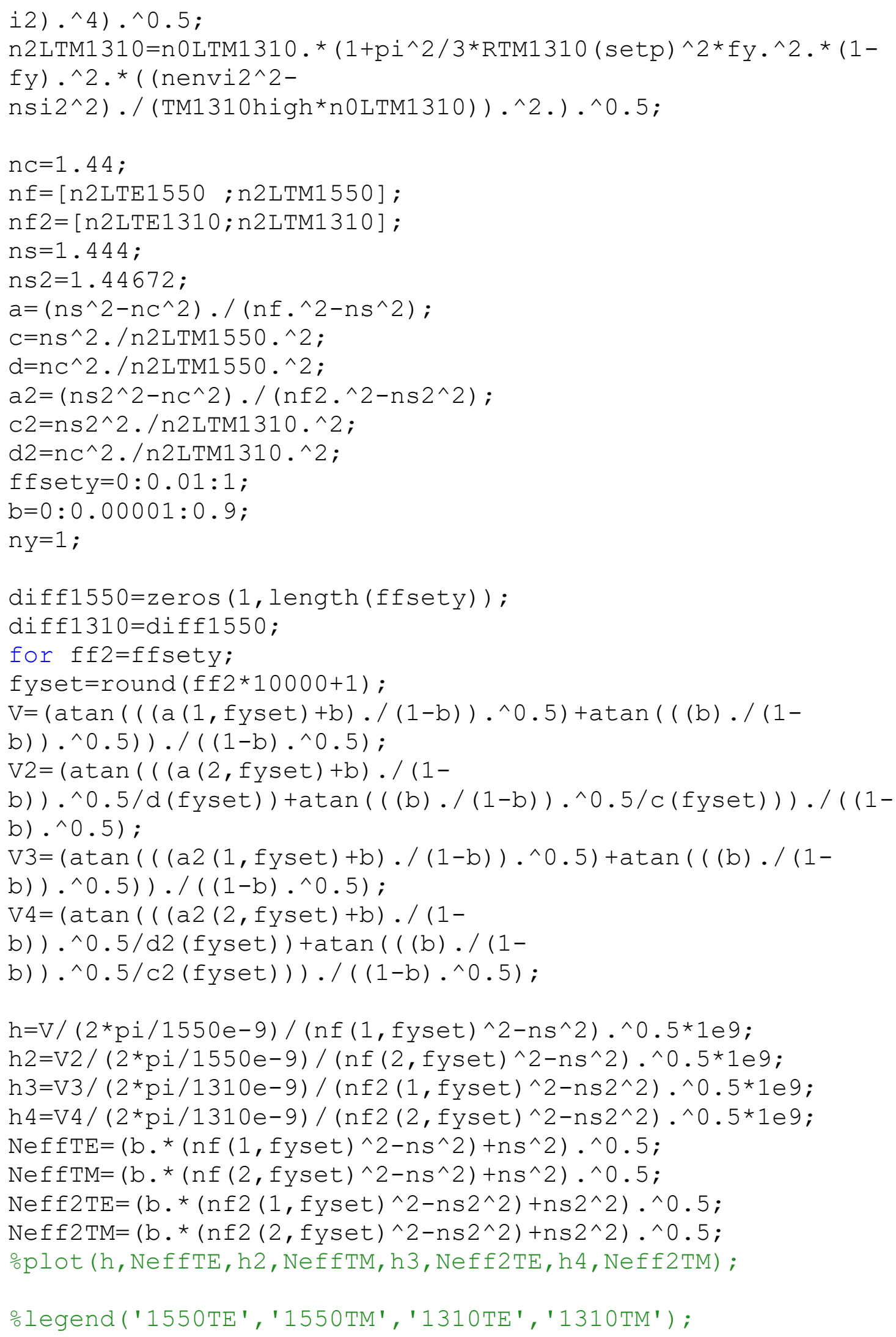




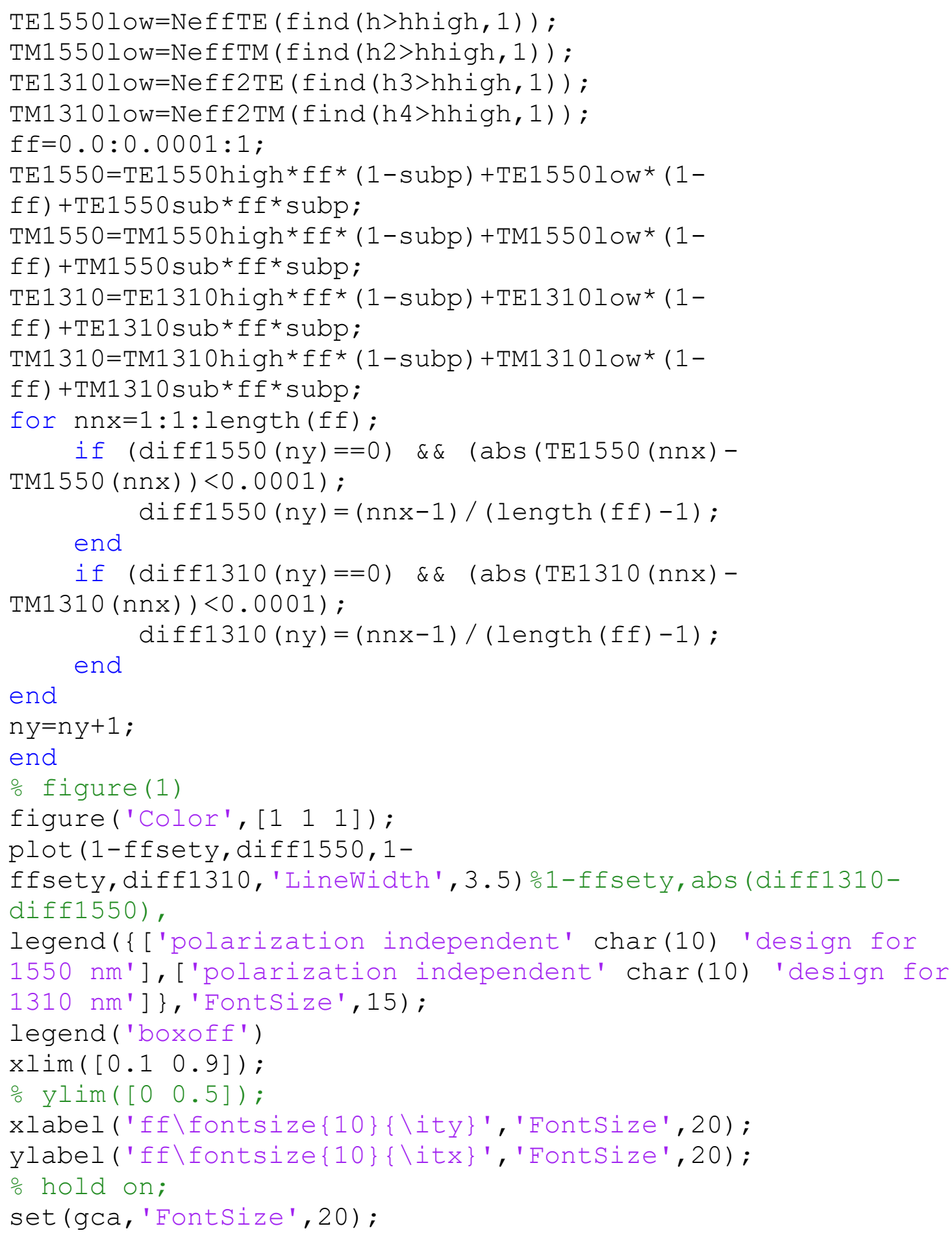

\section{B.5 Matlab code for Dual-Band Polarization Independent incident angle and}

\section{period length calculation}

clear all

load ('TEneff1310.mat'); 


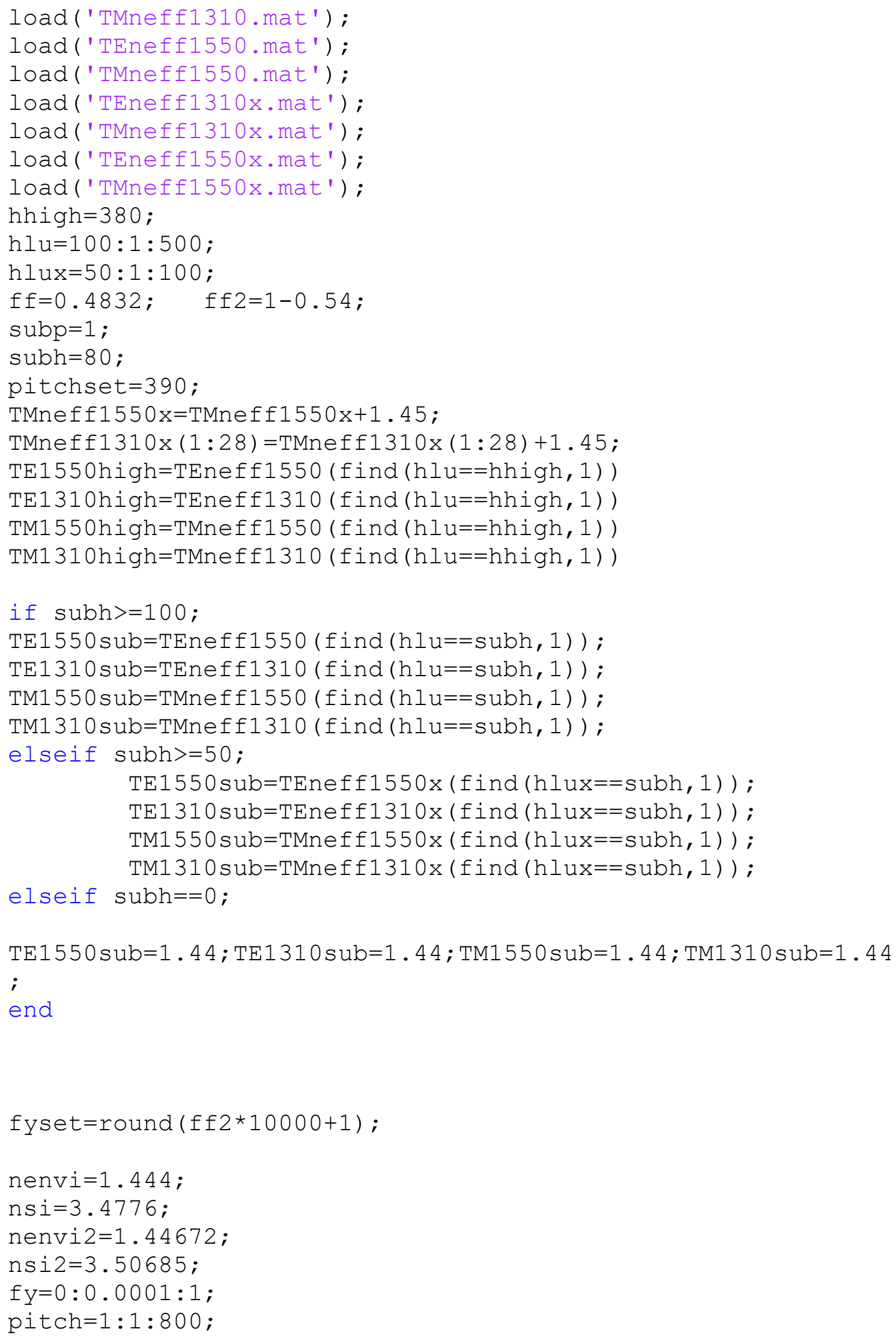




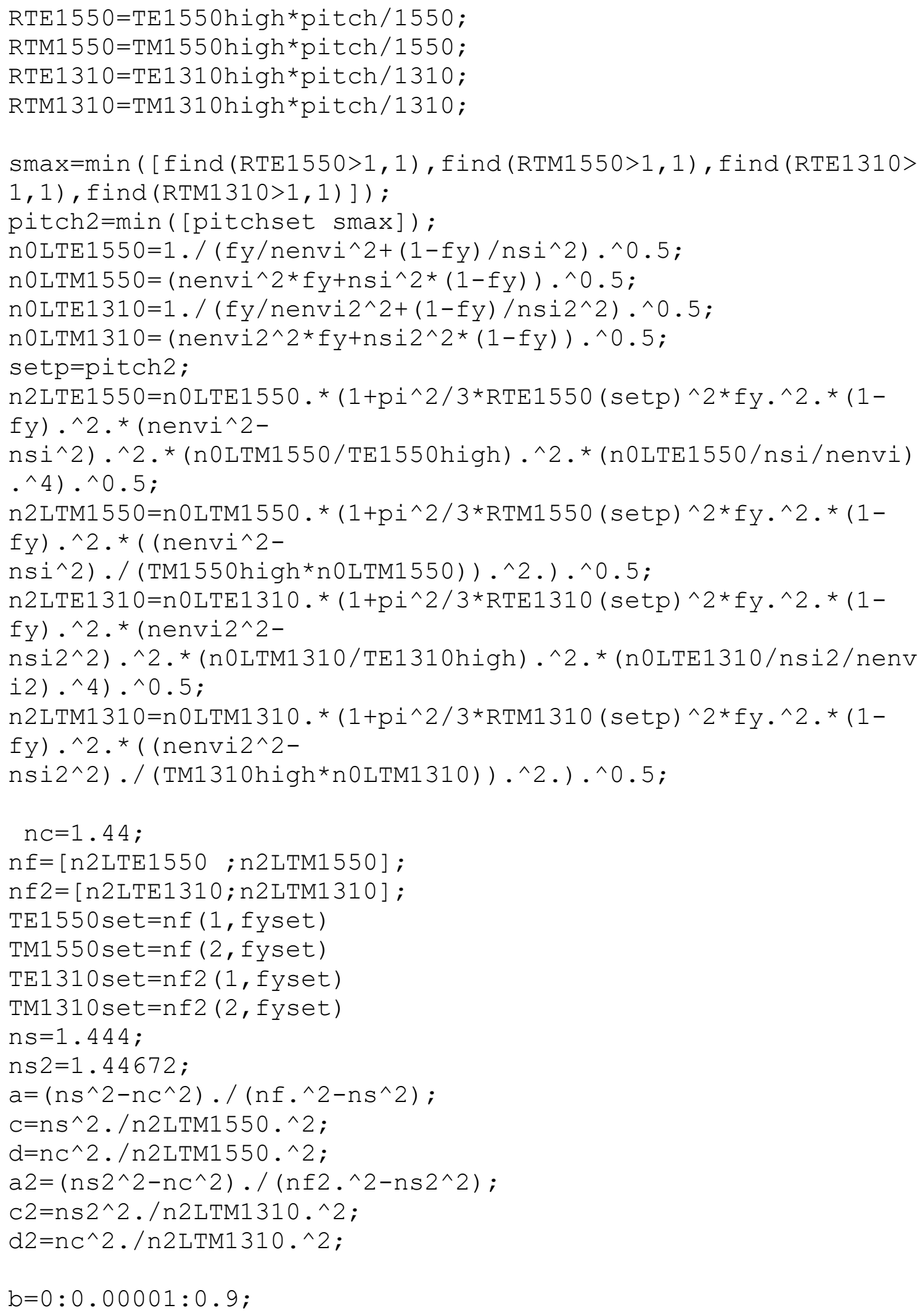




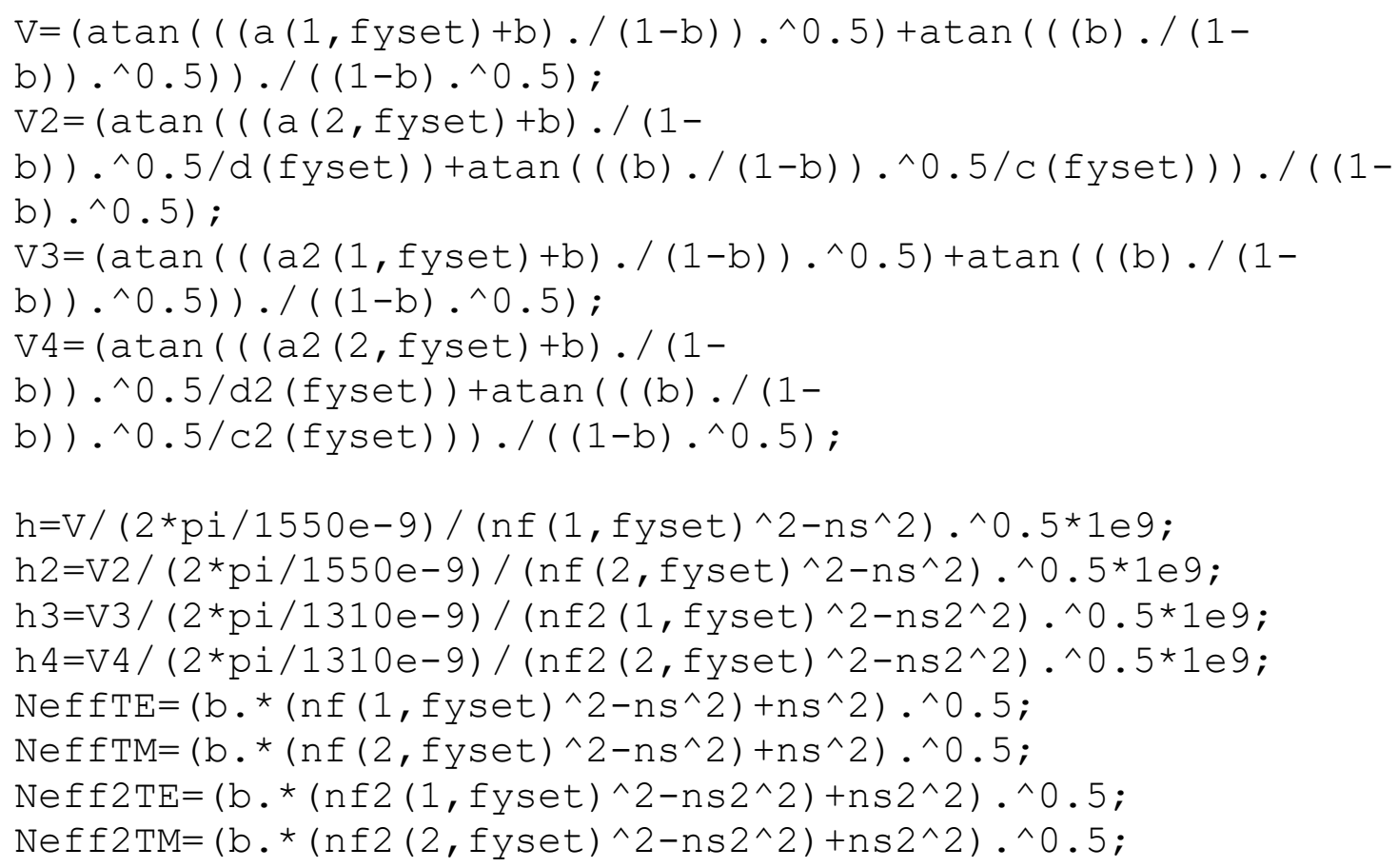


$\mathrm{y} 8=\operatorname{asin}((2 * 1310 . / \mathrm{n}-\mathrm{TM} 1310) /(-1.44672)) * 360 / 2 / \mathrm{pi}$;

figure (2)

clf

plot (n, abs (real (y1)), 'r', n, abs (real (y2)), 'b--

', n, abs (real (y3)), 'm', n, abs (real (y4)), 'c--

', 'LineWidth', 2.5) ;

$y \lim \left(\left[\begin{array}{ll}0 & 20\end{array}\right]\right)$; 


\section{Bibliography or References}

[1]. X. Chen et al., "The Emergence of Silicon Photonics as a Flexible Technology Platform," Proceedings of the IEEE, vol. 106, no. 12, pp. 2101-2116, 2018. Available: 10.1109/jproc.2018.2854372.

[2]. R. Wang, M. Muneeb, A. Vasiliev, A. Malik, S. Sprengel, G. Boehm, I. Simonyte, A. Vizbaras, K. Vizbaras, R. Baets, M.-C. Amann, and G. Roelkens, "III-V/silicon photonic integrated circuits for spectroscopic sensing in the $2 \mathrm{um}$ wavelength range," Smart Photonic and Optoelectronic Integrated Circuits XX, 2018.

[3]. A.Hornsteiner, "Fiber Optic Technology Trends in Data Transmission: Digitalization of data advance the need for constant upgrading of data networks," Optik \& Photonik, 12(4), 20-24. 2017.

[4]. S. Lee, K. Yasutomi, H. H. Nam, M. Morita, and S. Kawahito, "A Back-Illuminated Time-of-Flight Image Sensor with SOI-Based Fully Depleted Detector Technology for LiDAR Application,” Proceedings, vol. 2, no. 13, p. 798, 2018.

[5]. H. Rong, R. Jones, A. Liu, O. Cohen, D. Hak, A. Fang, and M. Paniccia, "A continuous-wave Raman silicon laser," Nature, vol. 433, no. 7027, pp. 725-728, 2005.

[6]. S. Nambiar, P. Sethi, and S. Selvaraja, "Grating-Assisted Fiber to Chip Coupling for SOI Photonic Circuits,” Applied Sciences, vol. 8, no. 7, p. 1142, 2018.

[7]. G. Son, S. Han, J. Park, K. Kwon, and K. Yu, "High-efficiency broadband light coupling between optical fibers and photonic integrated circuits," Nanophotonics, vol. 7, no. 12, pp. 1845-1864, 2018. 
[8]. Z. Cheng, X. Chen, C. Y. Wong, K. Xu, and H. K. Tsang, "Apodized focusing subwavelength grating couplers for suspended membrane waveguides," Applied Physics Letters, vol. 101, no. 10, p. 101104, Mar. 2012.

[9]. Yang and Z. Zhou, "Double-structure, bidirectional and polarization-independent subwavelength grating beam splitter," Optics Communications, vol. 285, no. 6, pp. 1494-1500, 2012.

[10]. P. J. P. Chausse, E. D. L. Boulbar, S. D. Lis, and P. A. Shields, "Understanding resolution limit of displacement Talbot lithography," Optics Express, vol. 27, no. 5, p. 5918, 2019.

[11]. S. Shao and Y. Wang, "Highly compact polarization-independent grating coupler," Optics Letters, vol. 35, no. 11, p. 1834, 2010.

[12]. R. Halir, P. Cheben, S. Janz, D. Xu, Í. Molina-Fernández and J. Wangüemert-Pérez, "Waveguide grating coupler with subwavelength microstructures," Optics Letters, vol. 34, no. 9, p. 1408, 2009. Available: 10.1364/ol.34.001408.

[13]. Z. Cheng, X. Chen, C. Y. Wong, K. Xu, C. K. Y. Fung, Y. M. Chen, and H. K. Tsang, "Mid-Infrared Grating Couplers for Silicon-on-Sapphire Waveguides," IEEE Photonics Journal, vol. 4, no. 1, pp. 104-113, 2012.

[14]. X. Chen and H. Tsang, "Nanoholes Grating Couplers for Coupling Between Silicon-on-Insulator Waveguides and Optical Fibers," IEEE Photonics Journal, vol. 1, no. 3, pp. 184-190, 2009.

[15]. C. Alonso-Ramos, X. L. Roux, J. Zhang, D. Benedikovic, V. Vakarin, E. DuránValdeiglesias, D. Oser, D. Pérez-Galacho, F. Mazeas, L. Labonté, S. Tanzilli, É. Cassan, D. Marris-Morini, P. Cheben, and L. Vivien, "Diffraction-less propagation 
beyond the sub-wavelength regime: a new type of nanophotonic waveguide," Scientific Reports, vol. 9, no. 1, 2019.

[16]. A. Mekis, S. Gloeckner, G. Masini, A. Narasimha, T. Pinguet, S. Sahni, and P. D. Dobbelaere, “A Grating-Coupler-Enabled CMOS Photonics Platform,” IEEE Journal of Selected Topics in Quantum Electronics, vol. 17, no. 3, pp. 597-608, 2011.

[17]. L. Yu, L. Liu, Z. Zhou, and X. Wang, "High Efficient Vertical Binary Blazed Grating Coupler for Chip Level Optical Interconnections,” Cleo, 2014.

[18]. X. Chen, C. Li, and H. K. Tsang, "Fabrication-Tolerant Waveguide Chirped Grating Coupler for Coupling to a Perfectly Vertical Optical Fiber,” IEEE Photonics Technology Letters, vol. 20, no. 23, pp. 1914-1916, 2008

[19]. L. Cheng, X. Mu, S. Wu, X. Tu, and H. Y. Fu, "Perfectly Vertical Grating Coupler for O and C-band," Frontiers in Optics Laser Science APS/DLS, 2019.

[20]. J. Tan, H. Pang, F. Meng, and J. Jiang, "Compact and high-efficient wavelength demultiplexing coupler based on high-index dielectric nanoantennas," Chinese Physics B, vol. 27, no. 9, p. 094217, 2018.

[21]. M. Streshinsky, R. Shi, A. Novack, R. T. P. Cher, A. E.-J. Lim, P. G.-Q. Lo, T. Baehr-Jones, and M. Hochberg, "A compact bi-wavelength polarization splitting grating coupler fabricated in a $220 \mathrm{~nm}$ SOI platform," Optics Express, vol. 21, no. 25, p. 31019 , Sep. 2013.

[22]. A. Y. Piggott, J. Lu, T. M. Babinec, K. G. Lagoudakis, J. Petykiewicz, and J. Vučković, "Inverse design and implementation of a wavelength demultiplexing grating coupler,” Scientific Reports, vol. 4, no. 1, 2014. 
[23]. G. Roelkens, D. V. Thourhout, and R. Baets, "Silicon-on-insulator ultra-compact duplexer based on a diffractive grating structure," Optics Express, vol. 15, no. 16, p. 10091, 2007.

[24]. L. Su, R. Trivedi, N. Sapra, A. Piggott, D. Vercruysse, and J. Vučković, "Fullyautomated optimization of grating couplers," Optics Express, vol. 26, no. 4, p. 4023, 2018. Available: $10.1364 /$ oe.26.004023.

[25]. J. Feng and Z. Zhou, "Polarization beam splitter using a binary blazed grating coupler,” Optics Letters, vol. 32, no. 12, p. 1662, May 2007.

[26]. Y. Tang, D. Dai, and S. He, "Proposal for a Grating Waveguide Serving as Both a Polarization Splitter and an Efficient Coupler for Silicon-on-Insulator Nanophotonic Circuits," IEEE Photonics Technology Letters, vol. 21, no. 4, pp. 242-244, 2009.

[27]. W. Bogaerts, D. Taillaert, P. Dumon, D. V. Thourhout, R. Baets, and E. Pluk, “A polarization-diversity wavelength duplexer circuit in silicon-on-insulator photonic wires,” Optics Express, vol. 15, no. 4, p. 1567, 2007.

[28]. D. Benedikovic, P. Cheben, J. H. Schmid, D.-X. Xu, B. Lamontagne, S. Wang, J. Lapointe, R. Halir, A. Ortega-Moñux, S. Janz, and M. Dado, "Subwavelength index engineered surface grating coupler with sub-decibel efficiency for 220 -nm silicon-oninsulator waveguides," Optics Express, vol. 23, no. 17, p. 22628, 2015.

[29]. R. Marchetti, C. Lacava, A. Khokhar, X. Chen, I. Cristiani, D. J. Richardson, G. T. Reed, P. Petropoulos, and P. Minzioni, "High-efficiency grating-couplers: demonstration of a new design strategy," Scientific Reports, vol. 7, no. 1, 2017.

[30]. D. Taillaert, Harold Chong, P. Borel, L. Frandsen, R. De La Rue, and R. Baets, "A compact two-dimensional grating coupler used as a polarization splitter," IEEE 
Photonics Technology Letters, vol. 15, no. 9, pp. 1249-1251, 2003. Available: 10.1109/lpt.2003.816671.

[31]. X. Chen and H. K. Tsang, "Polarization-independent grating couplers for siliconon-insulator nanophotonic waveguides," Optics Letters, vol. 36, no. 6, p. 796, Jul. 2011.

[32]. J. H. Song, F. E. Doany, A. K. Medhin, N. Dupuis, B. G. Lee, and F. R. Libsch, "Polarization-independent nonuniform grating couplers on silicon-on-insulator," Optics Letters, vol. 40, no. 17, p. 3941, 2015.

[33]. J. Zhang, J. Yang, H. Lu, W. Wu, J. Huang, and S. Chang, "Polarizationindependent grating coupler based on silicon-on-insulator," Chin. Opt. Lett. 13, 091301, 2015.

[34]. Z. Cheng and H. K. Tsang, "Experimental demonstration of polarization-insensitive air-cladding grating couplers for silicon-on-insulator waveguides," Opt. Lett. 39, 2206-2209, 2014.

[35]. W. Zhou, Z. Cheng, X. Sun, and H. K. Tsang, "Tailorable dual-wavelength-band coupling in a transverse-electric-mode focusing subwavelength grating coupler," Optics Letters, vol. 43, no. 12, p. 2985, 2018.

[36]. S. Nambiar, H. Muthuganesan, T. Sharma, and S. K. Selvaraja, "On-chip unidirectional dual-band fiber-chip grating coupler in silicon nitride," OSA Continuum, vol. 1, no. 3, p. 864, 2018.

[37]. W. Zhou and H. K. Tsang, "Dual-wavelength-band subwavelength grating coupler operating in the near infrared and extended shortwave infrared," Optics Letters, vol. 44, no. 15, p. 3621, 2019. 
[38]. F. W. Dabby, A. Kestenbaum, and U. C. Paek, "Periodic dielectric waveguides," Opt. Comm., Vol. 6, pp. 125-130 (1972).

[39]. S. Wang, "Principles of distributed feedback and distributed Bragg-reflector lasers," IEEE J. Quantum Electron., QE-10, No. 4, pp. 413-427 (1974).

[40]. A. Yariv, "Coupled-mode theory for guided-wave optics," IEEE J. Quantum Electron., Vol. QE-9, pp. 919-933 (1973).

[41]. D. Marcuse, Theory of Dielectric Optical Waveguides, Academic, New York, 1974, pp. $95-126,132-145$.

[42]. D. H. Raguin and G. M. Morris, "Antireflection structured surfaces for the infrared spectral region,” Applied Optics, vol. 32, no. 7, p. 1154, Jan. 1993.

[43]. R. Halir, P. J. Bock, P. Cheben, A. Ortega-Moñux, C. Alonso-Ramos, J. H. Schmid, J. Lapointe, D.-X. Xu, J. G. Wangüemert-Pérez, Í. Molina-Fernández, and S. Janz, "Waveguide sub-wavelength structures: a review of principles and applications," Laser \& Photonics Reviews, vol. 9, no. 1, pp. 25-49, 2014.

[44]. P. Cheben, R. Halir, J. Schmid, H. Atwater and D. Smith, "Subwavelength integrated photonics," Nature, vol. 560, no. 7720, pp. 565-572, 2018. Available: $10.1038 / \mathrm{s} 41586-018-0421-7$.

[45]. Y. Wang, W. Shi, X. Wang, Z. Lu, M. Caverley, R. Bojko, L. Chrostowski, and N. A. F. Jaeger, "Design of broadband subwavelength grating couplers with low back reflection," Optics Letters, vol. 40, no. 20, p. 4647, Aug. 2015.

[46]. Q. Zhong, V. Veerasubramanian, Y. Wang, W. Shi, D. Patel, S. Ghosh, A. Samani, L. Chrostowski, R. Bojko, and D. V. Plant, "Focusing-curved subwavelength grating 
couplers for ultra-broadband silicon photonics optical interfaces," Opt. Express 22, 18224-18231, 2014.

[47]. K.S. Chiang, "Analysis of optical fibers by the effective-index method", Applied Optics, 25(3):348-354, 1986.

[48]. K.S. Chiang. Effective-index method for the analysis of optical waveguide couplers and arrays: An asymptotic theory. Journal of Lightwave Technology, 9(1):62-72, 1991.

[49]. S. Rytov, "Electromagnetic properties of a finely stratified medium." Journal of Experimental and Theoretical Physics, 2, 466-475. ,1956

[50]. K. Yee, "Numerical solution of initial boundary value problems involving maxwells equations in isotropic media," IEEE Transactions on Antennas and Propagation, vol. 14, no. 3, pp. 302-307, 1966.

[51]. D. Taillaert, W. Bogaerts, P. Bienstman, T. Krauss, P. V. Daele, I. Moerman, S. Verstuyft, K. D. Mesel, and R. Baets, "An out-of-plane grating coupler for efficient butt-coupling between compact planar waveguides and single-mode fibers," IEEE Journal of Quantum Electronics, vol. 38, no. 7, pp. 949-955, 2002.

[52]. P. J. P. Chausse, E. D. L. Boulbar, S. D. Lis, and P. A. Shields, "Understanding resolution limit of displacement Talbot lithography," Optics Express, vol. 27, no. 5, p. 5918, 2019.

[53]. B. E. A. Saleh and M. C. Teich, Fundamentals of photonics. New York: Wiley, 1991.

[54]. "Overlap analysis - Modal Analysis Tab", Lumerical Support, 2020. [Online]. Available: https://support.lumerical.com/hc/en-us/articles/360034917453-Overlapanalysis-Modal-Analysis-Tab. [Accessed: 27- Jun- 2020]. 
[55]. C. Chen, Foundations for guided-wave optics. Hoboken, N.J.: Wiley-Interscience, 2007. 